\title{
POSSIBILISTIC INTERPRETATION OF MISTUNING IN BLADED DISKS BY FUZZY ALGEBRA
}

A THESIS SUBMITTED TO

THE GRADUATE SCHOOL OF NATURAL AND APPLIED SCIENCES $\mathrm{OF}$

MIDDLE EAST TECHNICAL UNIVERSITY

BY

HAMITT ÇAĞLAR KARATAŞ

IN PARTIAL FULLFILLMENT OF THE REQUIREMENTS FOR

THE DEGREE OF MASTER OF SCIENCE

IN

MECHANICAL ENGINEERING

SEPTEMBER 2012 
Approval of the thesis:

\section{POSSIBILISTIC INTERPRETATION OF MISTUNING IN BLADED DISKS BY FUZZY ALGEBRA}

submitted by HAMIT ÇAĞLAR KARATAŞ in partial fulfillment of the requirements for the degree of Master of Science in Mechanical Engineering Department, Middle East Technical University by,

Prof. Dr. Canan Özgen

Dean, Graduate School of Natural and Applied Sciences

Prof. Dr. Suha Oral

Head of Department, Mechanical Engineering

Prof. Dr. H. Nevzat Özgüven

Supervisor, Mechanical Engineering Dept., METU

Asst. Prof. Dr. Ender Ciğeroğlu

Co-Supervisor, Mechanical Engineering Dept., METU

Examining Committee Members

Prof. Dr. Mehmet Çalışkan

Mechanical Engineering Dept., METU

Prof. Dr. H. Nevzat Özgüven

Mechanical Engineering Dept., METU

Asst. Prof. Dr. Ender Ciğeroğlu

Mechanical Engineering Dept., METU

Asst. Prof. Dr. Yiğit Yazıcıoğlu

Mechanical Engineering Dept., METU

Asst. Prof. Dr. M. Bülent Özer

Mechanical Engineering Dept., TOBB-ETU

Date:

21.09 .2012 
I hereby declare that all information in this document has been obtained and presented in accordance with academic rules and ethical conduct. I also declare that, as required by these rules and conduct, I have fully cited and referenced all material and results that are not original to this work.

Name, Last name : Hamit Çağlar KARATAŞ

Signature : 


\begin{abstract}
POSSIBILISTIC INTERPRETATION OF MISTUNING IN BLADED DISKS BY FUZZY ALGEBRA

Karataş, Hamit Çağlar

M.S., Department of Mechanical Engineering

Supervisor: $\quad$ Prof. Dr. H. Nevzat Özgüven

Co-supervisor: $\quad$ Asst. Prof. Dr. Ender Ciğeroğlu
\end{abstract}

September 2012, 103 pages

This study aims to define the possibilistic interpretation of mistuning and examine the way of determining the worst case situations and assessing reliability value to that case by using possibilistic methods. Furthermore, in this study, benefits of using possibilistic interpretation of mistuning in comparison to probabilistic interpretation of mistuning are investigated.

For the possibilistic analysis of mistuned structures, uncertain mistuning parameters are modeled as fuzzy variables possessing possibility distributions. In this study, alpha-cut representations of fuzzy numbers are used which makes fuzzy variables to be represented by interval numbers at each and every confidence level. The solution of fuzzy equations of motion is governed by fuzzy algebra methods. The bounds of the solution of the fuzzy equation of motion, i.e. fuzzy vibration responses of the mistuned structure, are determined by the extension principle of fuzzy functions.

The performance of the method for possibilistic interpretation of mistuning is investigated by comparing it to the probabilistic methods both computational and accuracy wise. For the comparison study, two different optimization tools - genetic 
algorithm as the global optimization tool and constrained nonlinear minimization method as the gradient based optimization tool- are utilized in possibilistic analysis and they are compared to solutions of probabilistic methods resulted from MonteCarlo method. The performances of all of the methods are tested on both a cyclically symmetric lumped parameter model and a realistic reduced order finite element model.

Keywords: $\quad$ Vibrations of Mistuned Bladed Disks, Theory of Possibility, Fuzzy Algebra, Maximum Amplification Factor, Worst Mistuning Pattern. 


\title{
$\ddot{O Z Z}$
}

\section{DİSK-KANATÇIK SİSTEMLERİNDEKİ DÜZENSİZLİK SORUNUNUN BULANIK CEBİR KULLANILARAK OLABİLIRLİKSEL İNCELEMESİ}

\author{
Karataş, Hamit Çağlar
}

\author{
Yüksek Lisans, Makine Mühendisliği Bölümü \\ Tez Yöneticisi: $\quad$ Prof. Dr. H. Nevzat Özgüven \\ Ortak Tez Yöneticisi: $\quad$ Yrd. Doç. Dr. Ender Ciğeroğlu
}

Ekim 2012, 103 sayfa

Kanatçıklardaki eşsizlik sorununun olabilirliksel incelemesi için belirsiz etmenler olabilirliksel dağılım gösteren bulanık etmenlerle açıklanmıştır. Olabilirliksel dağılımlar alfa kesitleri tanımları kullanılarak güvenilirlik seviyelerine ayrılarak incelenmiş ve her seviye bir aralık sayısı ile gösterilmiştir. Bulanık titreşim cevabı değişkenlerinin çözümünde bağımlılık sorununa yol açan aralık cebiri yöntemleri yerine genişletme yöntemi kullanılmış ve elde edilen sonuçların daha doğru olması sağlanmıştır. Kanatçıklardaki eşsizlik sorunun olabilirliksel olarak ele alınmasının örneklenmesi için kullanılan bulanık etmenleri en fazla $\pm \% 5$ değişebilen çevresel simetrik noktasal yapının çözümünde genişletme yöntemi kullanılmıştır. Bu yöntem kullanılarak ortaya çıkabilecek en kötü durumun belirlenebilmesi için değişim eniyileme algoritmaları kullanılarak yeni bir yöntem geliştirilmiştir.

Ortaya konulan kanatçıklı disklerdeki eşsizlik sorunun olabilirliksel olarak ele alan yöntemin başarımı, onun doğruluk ve hesaplama zamanı açısından olabilirliksel yöntemlerle karşılaştırılması ile sınanmıştır. Karşılaştırma çalışması için genişletme 
yöntemi çözümünde hem genetik algoritma hem de değişim eniyileme algoritmaları ile çözülmüş ve sonuçlar olabilirliksel yöntem olarak temel Monte-Carlo yöntemleri ile karşılaştırılmıştır. Tüm başarım sınamaları çevresel simetrik noktasal yapı ve daha gerçekçi indirgenmiş sonlu elemanlar modeli üzerinden gerçekleştirilmiştir.

Anahtar Kelimeler: Düzensiz Kanatçıklı Disklerin Titreşimi, Olabilirlik Teoremi, Bulanık Cebir, Maksimum Yükseltme Katsayısı, En Kötü Düzensizlik Durumu. 
To My Dearest Family and To Charm of Science and Knowledge 


\section{ACKNOWLEDGEMENTS}

I am honored to pursue this research study with my supervisor Prof.Dr. H. Nevzat ÖZGÜVEN and Asst. Prof. Dr. Ender CİĞEROĞLU. I would like to thank to them to share their technical profession, experience and academic perfection.

I feel myself so lucky to have such a family. I would like to express my deepest appreciation to them all for their continuous support and love. 


\section{TABLE OF CONTENTS}

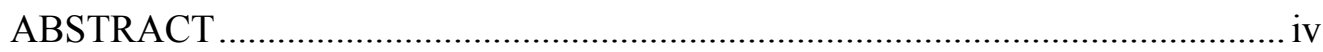

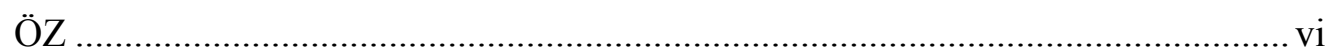

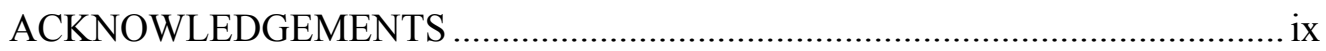

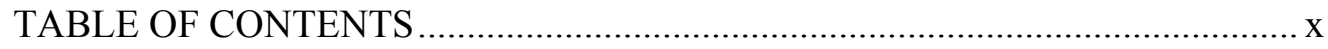

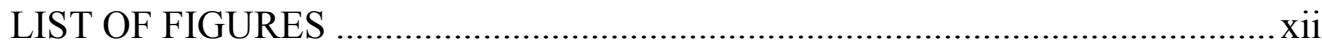

CHAPTERS

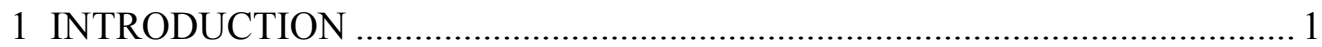

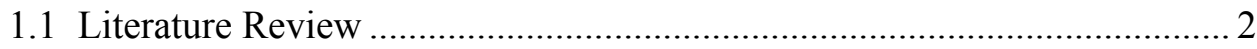

1.2 Objectives of the Thesis ................................................................... 5

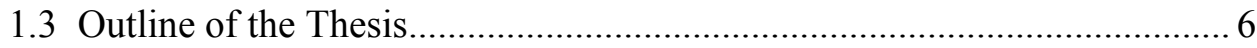

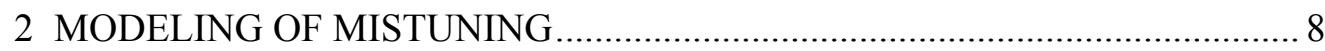

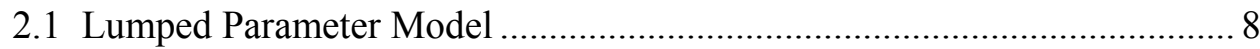

2.2 Reduced Order Finite Element Model................................................... 11

2.2.1 Fixed Interface Modal Coupling Method ....................................... 11

3 UNCERTAINTY QUANTIFICATION IN MISTUNING ............................ 18

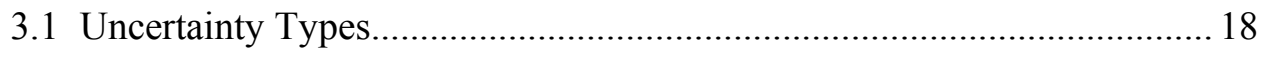

3.2 Uncertainty Management Methods ........................................................ 19

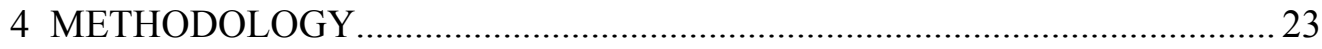




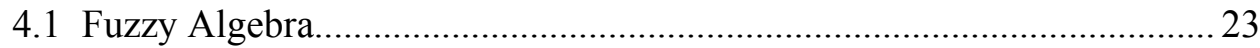

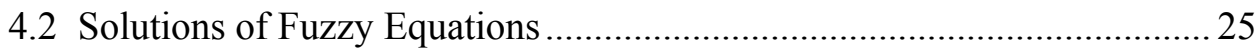

4.3 Fuzzy Forced Response Analysis ....................................................... 26

4.4 Use of Optimization Methods on Solutions to Fuzzy Dynamic Equations28

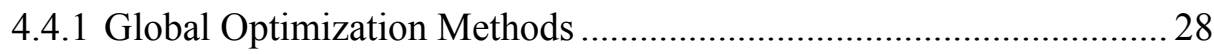

4.4.2 Gradient-Based Optimization Algorithm ....................................... 28

4.4.3 Types of Next Level of Confidence and Initial Guess Determination

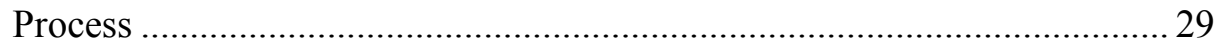

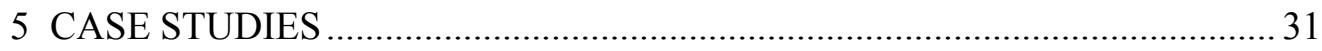

5.1 CASE I - Possibilistic Interpretation of Mistuning ............................... 31

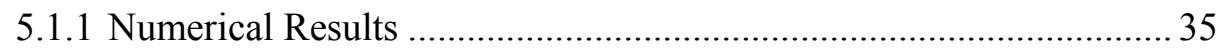

5.2 CASE II - Comparison between Uncertainty Management Methods....... 38

5.2.1 Numerical Examples ............................................................... 42

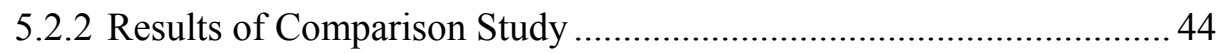

5.3 CASE III - Comparison between Methods in the Determination of the

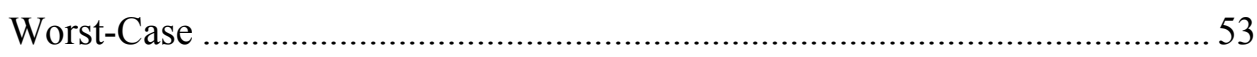

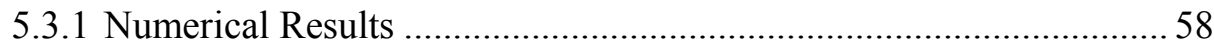

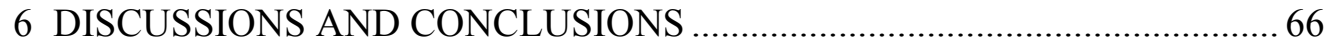

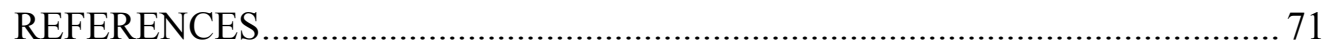

APPENDIX

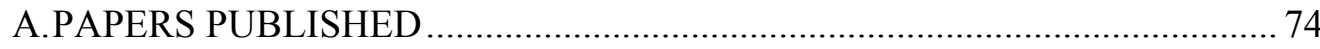




\section{LIST OF FIGURES}

\section{FIGURES}

Figure 2.1 : Cyclically symmetric lumped-parameter jet-engine blisk model [22] ..... 9

Figure 2.2 : Representation of double modes of tuned structure and corresponding mode shapes with phase difference only

10

Figure 2.3 : Representation of mode splitting of double modes and corresponding energy localization phenomenon occurring in mode shape. .................................. 10

Figure 3.1: A triangular membership function distribution ................................... 21

Figure 5.1 : Possibility distribution for disk stiffness parameters............................ 33

Figure 5.2: Low, moderate and high risk level assessments .................................... 34

Figure 5.3 : Displacement of the blade corresponding to the maximum response vs.

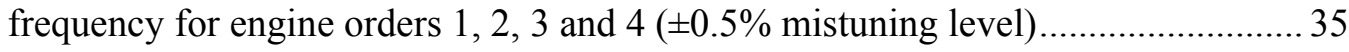

Figure 5.4 : Maximum response of blades for engine orders 1, 2, 3 and $4( \pm 5 \%$

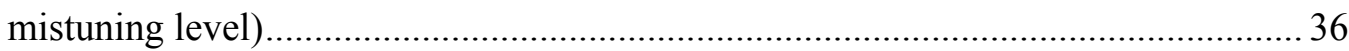

Figure 5.5: Consistency of the two uncertainty models........................................... 39

Figure 5.6: Alpha-cuts of possibility distributions................................................ 40

Figure 5.7: 10 dof lumped parameter model ......................................................... 43

Figure 5.8 : Simple lumped parameter system $4^{\text {th }}$ natural frequency distribution- first

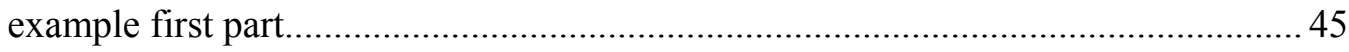

Figure 5.9 : Simple lumped parameter system $3^{\text {rd }}$ natural frequency distributions -

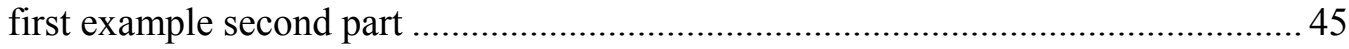

Figure 5.10 : Computational efficiency comparison - First example first part ......... 46

Figure 5.11 : Computational efficiency comparison - First example second part..... 48

Figure 5.12 : Mistuned bladed disk model 9th natural frequency distribution .......... 51

Figure 5.13 : Computational efficiency comparison - Second example .................. 51

Figure 5.14: Fundamental sector geometry [24] ................................................. 56

Figure 5.15: The bladed-disk geometry [24] ….................................................. 56

Figure 5.16: Maximum blade vibration values for all possible mistuning pattern configurations of blade \#1 and blade $\# 19$............................................................ 58 
Figure 5.17: Visualization of the first initial guess and next level of confidence

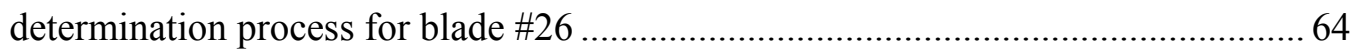
Figure 5.18: Visualization of the second initial guess and next level of confidence

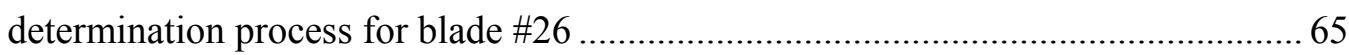




\section{CHAPTER 1}

\section{INTRODUCTION}

Gas turbine engines are composed of cyclically symmetric machine parts such as, fans, compressors and turbines, all of which are referred as bladed disks or blisks. These structures are designed to be constructed by repetition of a fundamental sector around the circumference; therefore, in design, all sectors are assumed to be completely identical. In this case the structure is termed as 'tuned' structure. However, in reality, due to deviation in structural properties between repeating sectors - that may occur due to manufacturing tolerances, material non-uniformities or even from service wear - the cyclic symmetry is destroyed. In literature, the structure which has lost its cyclic symmetry is named as 'mistuned' structure and the phenomenon related to the consequences in the vibratory behavior of the structure is called 'mistuning'.

For a tuned structure, using cyclic symmetry properties, determination of the vibratory behavior of the structure is a straight forward process where only the information of a single sector and the related cyclic boundary conditions are sufficient. However, the studies on mistuning show that for the mistuned system operating in the same conditions with the tuned counterpart, due to the destruction of the cyclic symmetry, there exists possibility of vibration energy localization on a particular blade resulting in significant increase in blade vibration amplitudes. Consequently, the increase of vibration amplitude results in unexpected decrease in actual high cycle fatigue life of a blade. Therefore, mistuning phenomenon is one of the crucial topics in the gas turbine industry, which have been investigated in several aspects. 
In gas turbine engines, in order to come up with a reliable design, consequences of mistuning need to be both determined or compensated prior to service. The performances of the methods utilized for determination or compensation of possible effects of mistuning are based on correct treatment of uncertainties in the structure. Up to now, these uncertainties are handled by probabilistic methods and in this thesis the benefits of using possibilistic methods on quantifying uncertainties in mistuning studies are investigated.

\subsection{Literature Review}

Mistuning is a well-studied phenomenon that has already been divided into several branches such as; identification, forced response reduction, health monitoring, worst/best scenario or mistuning pattern determination. Hence up to now, researchers tried to identify a present mistuning, tried to reduce the level of amplification factor for a given design, monitor the amplification factor as the bladed disk operates or tried to determine the worst and the best possible cases within a given uncertainty interval prior to service. Among all these efforts, determining the worst/best case study is the most suitable for the possibilistic analysis to be effective on, since possibilistic analysis is an uncertainty quantification tool that quantifies levels of confidences of all possible cases. In this thesis, for reliability considerations, in contrast to present methods that utilize probabilistic analysis, determining the worstcase situations are investigated by using possibilistic analysis that employs fuzzy algebra

The two methods present to handle uncertainties - probabilistic and possibilistic methods - can be used interchangeably regarding possibility/probability consistency theorem. There are several studies done in the literature that tries to determine the favorable method for different types of engineering problems which are reviewed by Maglaras et.al [1]. In their review paper, the authors state the common conclusion in literature that probabilistic methods favor in situations where there is enough 
information regarding uncertainties, and possibilistic methods favor in situations where there is lack of information and in situations where the worst-case scenarios are sought. Therefore it is logical to conduct a research on mistuning worst-case determination problem that looks for the worst-possible situations by utilizing possibilistic methods rather than probabilistic methods.

In literature, in order to determine the worst-mistuning patterns and consequently the worst case mistuning amplification factors, excluding the initial analytical works done mainly by Whitehead [2], basic Monte-Carlo methods are used [3,4,5]. In these studies, a number of random mistuning patterns are iterated by Monte-Carlo methods and consequently a random set for mistuning amplification factor data is collected. The worst-case situation for the bladed disk/blisk considered is set to be the case where maximum amplification factor value is converged as the number of iterations is increased. The probability density functions are determined by ranking frequencies of occurrences of amplification factors calculated and a probability value is assigned to the worst-case situation by using the calculated probability density function. However, the worst-case amplification factor results are observed to be much smaller than the Whitehead theoretical formula [5] and this method has a temporal deficiency, since large bladed disk finite element models are needed to be iterated in this method.

The definition of the worst-case situations has been changed after formulating it as an optimization problem $[3,6,7]$. In these studies, uncertain variables are modeled as random variables with known distributions. The random sets that are characterized by corresponding distributions are used as the inputs of the optimization problem. The transfer function defined by the vibratory characteristics of the structure at hand maps the probable combinations of input space to the space of the probable outputs. The worst-case situation is defined to be the combination of input parameter values that results in the highest amplification factor value in the output space. The search of the highest amplification factor value in the space is done by either global optimization methods, gradient-based optimization methods or a combination of both. In literature, no earlier studies have been observed than the study of Petrov in 
1988 [6] and in 1993 [7] that formulates the worst-case problem in mistuning studies. Afterwards, Petrov also studied the problem using sensitivity coefficients [8] and genetic algorithms using gradient-based response surface approximations [9]. In 2005, Scarcelli and Leece used genetic algorithms and neural networks [10]. Petrov and Ewins analyzed the worst mistuning patterns in bladed disk assemblies in 2003 [3] by gradient-based quasi-Newton optimization methods. In this study, authors used the method proposed by Petrov, Sanliturk and Ewins [11] that is based on exact relationship between tuned and mistuned assemblies using Sherman-MorrisonWoodbury formula. This method enabled the use of realistic finite element bladed disk models and authors identified the maximum amplification factors of a fan bladed disk with 24 blades and a high-pressure turbine bladed disk with 92 shrouded blades and achieved to gather an amplification factor so close to the Whitehead theoretical formula. The authors used Monte-Carlo simulations in order to show the efficiency of the methods in comparison with the similar studies and they have stated the results as global by checking the derivative of the sensitivity coefficients to be zero. In a more recent paper, it is possible to observe similar finite element modeling types of bladed disks that utilizes Sherman-Morrison-Woodbury formula but with different optimization strategies; genetic algorithm and a hybrid GA-SQP algorithm [12]. In these studies, the amplification factor values turned out to be below from the Whitehead theoretical formula and the case studies were done by lumped-parameter models.

For the reliability considerations the aim is not only to find the worst-case scenarios but to assess a level-of-confidence or probability of occurrence to it. Nevertheless, none of the studies mentioned above have proposed a probability value to the calculated results. However, in the doctorate dissertation that examines the variability of blade vibration in bladed disks, Chan [5] proposed a method to assess a probability value to the calculated optimization worst case results. In the proposed method, first, a small Direct-Monte-Carlo run is done in order to approximate to the probability density function of the uncertain amplification factors. Then global optimum results are found by conjugate gradient method. Finally, the probability of 
the worst result is assessed after the curve-fitting procedure to the line that is constructed by importance sampling method. Therefore, in order to come-up with the probability of the worst result a four-stage method needed to be pursued which contains two probabilistic methods, one optimization method.

In contrast, in this thesis, reliability levels of all possible outputs are determined by assessing corresponding possibility values using possibility theorem [13]. The possibility theorem is an alternative tool for handling uncertainties that is used in situations where the information is ambiguous, vague or imprecise. In possibilistic analysis, uncertain parameters are modeled as fuzzy variables characterized by possibility distributions and hence, crisp dynamic equations become fuzzy dynamic equations. For vibration studies, obtaining fuzzy natural frequencies, fuzzy mode shapes, fuzzy forced responses and fuzzy FRF's are important. These parameters are calculated using any of the methods like interval arithmetic methods $[14,15,16,17$, 18, 19], optimization methods [20] or neural networks [21]. The first paper on the solution of fuzzy dynamic equations in the field of fuzzy finite element analysis was proposed by Chan and Rao at 1997 [16]. In this paper, authors proposed Taguchi robust technique in order to determine boundaries of possible distributions. The interval arithmetic method turned out to be the fastest method but this method also resulted in over-conservative results due to the dependency problem. The efficiencies of the rest of the methods differ with respect to aim and type of the problem.

\subsection{Objectives of the Thesis}

In this thesis, the aim is to propose a new method using possibility theorem for the solution to the problem of determining the worst-case situations within mistuning uncertainty limits and assessing a level-of-confidence to the worst-possible result for the reliability considerations. In other words, this study aims:

1. To define possibilistic interpretation of mistuning 
2. To propose a fast and accurate method for determining the highest vibration level in a mistuned bladed disk.

3. To assess a level-of-confidence value to all possible forced response values.

\subsection{Outline of the Thesis}

This thesis is organized in six chapters for efficient explanation of mistuning phenomenon, uncertainty quantification methods in mistuning, methodology of possibilistic interpretation of mistuning, numerical cases used for exemplification and evaluation of the proposed method from accuracy and computational efficiency points of view; interpretation of the results of the numerical case studies and concluding remarks.

Chapter 2 introduces the types of mistuned bladed disk models. The basic concepts related to mistuning studies and types of mistuned bladed disk models that are used in this thesis are detailed. The basic vibratory properties of mistuned bladed disk assemblies are explained by using the dynamic models presented.

Chapter 3 states the theorems and methods used for uncertainty quantification. Types of uncertainties are defined and the two approaches to handle uncertain situations in engineering systems - possibility and probability theorems - are detailed. Two methods are further clarified by stating similarities and differences among each other.

Chapter 4 details the required background information for possibilistic interpretation of mistuning. The uncertainties present in mistuned bladed disks are treated as imprecise, and modeled as fuzzy variables. Basic algebraic operations on fuzzy variables are introduced. The definition of fuzzy functions and fuzzy equations are given and solution of fuzzy equations is stated in detail. Also this chapter explains fuzzy modal analysis that is required to determine fuzzy forced responses of the mistuned bladed disk systems. 
Chapter 5 contains numerical case studies that are constructed for exemplification and evaluation of the method proposed in this thesis. First, a case study is constructed for exemplification of the possibilistic interpretation of mistuning. In this case study, the worst-possible situation of a lumped parameter bladed disk model in each and every confidence level is sought. The mistuning parameters are assumed to be deviating $\pm 5 \%$ at most and the amplification factor values for low, moderate and high mistuning levels are determined. In the second case study, the same model is further used for evaluation of efficiency of the possibilistic method by comparing computational time and accuracy of probabilistic and possibilistic methods on the same mistuning problem. Finally, the third case study is built up for testing the success of the method in determining the worst-possible situations using a realistic reduced order finite element model. The same mistuning situation is solved by both hybrid optimization methods and possibilistic methods for determining the worstpossible amplification factor values.

Chapter 6 makes discussions on the results gathered and draws the conclusions gained out of the study. Furthermore, suggestions on future work and further developments are given. 


\section{CHAPTER 2}

\section{MODELING OF MISTUNING}

Bladed disk systems are cyclically symmetric systems which can be modeled by lumped parameter models or detailed finite element models. Lumped parameter models are theoretical models of bladed disks that represent the basic vibratory properties of real bladed disk systems. These models require detailed modeling efforts for determination of system parameters and therefore have limited use. Finite element models contain on the order of million DOFs which limits its direct use in design optimization. Instead of using whole system matrices of finite element models, reduced order models (ROMs) of the systems are utilized for design optimization. In this study, both a cyclically symmetric lumped parameter model and a reduced order finite element model are utilized for exemplification and evaluation of the possibilistic interpretation of mistuning and for comparison studies between possibilistic and probabilistic methods.

\subsection{Lumped Parameter Model}

A lumped parameter model of a bladed disk system is given in Figure 2.1 which is composed of 16 repeating sectors, where $M, m, k_{d}, k_{b}, h_{d}$ are the disk mass, blade mass, disk stiffness, blade stiffness and structural damping, respectively. 


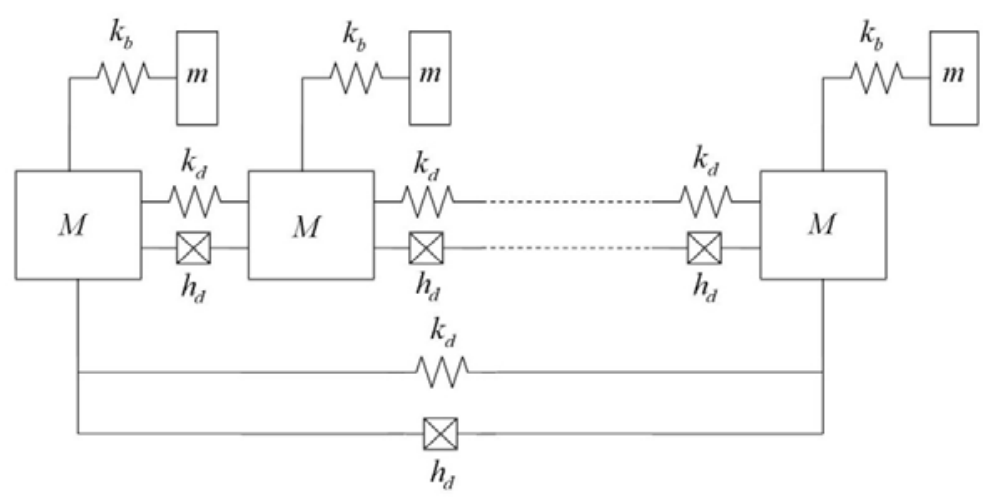

Figure 2.1 : Cyclically symmetric lumped-parameter jet-engine blisk model [22]

As stated before, lumped parameter models are theoretical models that can model the vibratory behavior of the complicated bladed disk systems. Due to the nature of cyclic symmetry, tuned bladed disk structures have double modes that have the same natural frequency value with two different mode shapes oriented with a phase difference. The double modes and mode shapes of a representative bladed disk model are shown in Figure 2.2. For a mistuned bladed disk structure, double modes separate and there exists possibility of energy localization as shown in Figure 2.3.

Depending on the type of mistuning, the choice of mistuning parameter might change in lumped parameter models. It is possible to reflect mistuning on stiffness, mass or damping parameters as deviations from the design value or combinations of those. 
Figure 2.2 : Representation of double modes of tuned structure and corresponding mode shapes with phase difference only

Figure 2.3 : Representation of mode splitting of double modes and corresponding energy localization phenomenon occurring in mode shape. 


\subsection{Reduced Order Finite Element Model}

Bladed disk systems are cyclically symmetric systems that are formed by repetition of the fundamental sector around the rotation axes. Likewise, finite element model of the bladed disk systems are constituted by coupling the repetitive sectors with each other by using either modal or spatial coupling techniques around the rotation axes. However, the consequent bladed disk finite element model may contain millions of degrees of freedom; hence using the model in modal analysis to determine its dynamic behavior is computationally expensive. Consequently, reduced order finite element models are used which are accurate enough to show the dynamic behavior of the whole bladed disk system for the modes considered. In this study, by considering cyclic symmetry property of bladed disks, fixed interface modal coupling method or commonly known as Craig-Bampton method - is utilized to couple blade and disk substructures and to obtain reduced order finite element model.

\subsubsection{Fixed Interface Modal Coupling Method}

In the article of Young [23], the basics and the uses of Craig-Bampton method are explained in detail. Orbay [24] summarizes the use of the method for modal coupling and refers to the study of Bladh [25] for the implementation of the method for bladed disk models. In this thesis, only the basics of the Craig-Bampton method that are required for explaining mistuning modeling is presented, and the reader is referred to aforementioned studies [23, 24, 25] for further details.

In the present study, the fixed interface modal coupling method is used for modal synthesis of substructures that have common interfaces. The substructures are partitioned, reduced and coupled to perform an efficient modal analysis of the larger sized combined structure by using modal information of smaller sized substructures. In this section, all these processes are detailed in the given order. 
First of all, in fixed base component mode synthesis method, coordinates of each substructure are separated into interface (boundary) and elastic coordinates;

$$
\{U\}=\left\{\begin{array}{l}
\left\{U_{L}\right\} \\
\left\{U_{R}\right\}
\end{array}\right\}
$$

where $\left\{U_{L}\right\}$ stands for vector of elastic coordinates and $\left\{U_{R}\right\}$ stands for vector of boundary coordinates. Partitioning physical degrees of freedom results in corresponding partitioning of system matrices; thus, equation of motion transforms into the following form

$$
\left[\begin{array}{ll}
{\left[M_{L L}\right]} & {\left[M_{L R}\right]} \\
{\left[M_{R L}\right]} & {\left[M_{R R}\right]}
\end{array}\right]\left\{\begin{array}{l}
\left\{\ddot{U}_{L}\right\} \\
\left\{\ddot{U}_{R}\right\}
\end{array}\right\}+\left[\begin{array}{ll}
{\left[K_{L L}\right]} & {\left[K_{L R}\right]} \\
{\left[K_{R L}\right]} & {\left[K_{R R}\right]}
\end{array}\right]\left\{\begin{array}{c}
\left\{U_{L}\right\} \\
\left\{U_{R}\right\}
\end{array}\right\}=\left\{\begin{array}{l}
\left\{f_{L}\right\} \\
\left\{f_{R}\right\}
\end{array}\right\}
$$

Secondly, in this method, in order to reduce the size of large finite element models, the elastic coordinates are transformed into a set of modal coordinates and truncation is made. The system matrices in modal coordinates contain modal properties of the substructure whose boundary coordinates are fixed and only the modes of interest are taken into account by truncating higher order elastic modes of vibration. On the contrary, boundary nodes stay in physical coordinates and they are reserved for coupling between substructures. The transformation from physical to modal elastic coordinates is done by Craig-Bampton transformation matrix that is composed of boundary node functions and fixed base mode shapes as follows:

$$
\left\{\begin{array}{c}
\left\{U_{L}\right\} \\
\left\{U_{R}\right\}
\end{array}\right\}=[T]\left\{\begin{array}{c}
\left\{q_{m}\right\} \\
\left\{U_{R}\right\}
\end{array}\right\}=\{[\Phi] \quad[B]\}\left\{\begin{array}{c}
\left\{q_{m}\right\} \\
\left\{U_{R}\right\}
\end{array}\right\}=\left\{\left[\begin{array}{c}
{\left[\Phi_{\mathrm{L}}\right]} \\
{[0]}
\end{array}\right]\left[\begin{array}{c}
{\left[\Phi_{\mathrm{R}}\right]} \\
{[I]}
\end{array}\right]\right\}\left\{\begin{array}{c}
\left\{q_{m}\right\} \\
\left\{U_{R}\right\}
\end{array}\right\}
$$


In Equation (2.3), $[T]$ is the transformation matrix, $[\Phi]$ is the matrix of fixed base mode shapes and $[B]$ is the matrix of boundary node functions. From Equation (2.3) it is evident that physical displacements of elastic coordinates can be computed as,

$$
\left\{U_{L}\right\}=\left[\phi_{L}\right]\left\{q_{m}\right\}+\left[\phi_{R}\right]\left\{U_{R}\right\}
$$

where $\left[\phi_{R}\right]\left\{U_{R}\right\}$ represents rigid body displacements of elastic coordinates due to boundary coordinates and $\left[\phi_{L}\right]\left\{q_{m}\right\}$ represents displacements of elastic degrees of freedom relative to the displaced base [23]. In order to determine the matrix $\left[\phi_{R}\right]$, initially, all the degrees of freedom of boundary nodes should be fixed and then structural displacements of the substructure should be solved for the unit displacement of consecutively released degrees of freedom. The matrix $\left[\phi_{L}\right]$ is composed of eigenvectors of the unforced harmonic motion of the fixed base structure [23]. Since the remaining elements of the transformation matrix are zero and unity matrices, calculation of matrix of fixed base mode shapes and matrix of boundary node functions is enough to calculate the transformation matrix.

Transformation of the equation of motion from physical coordinates to a hybrid set of physical and modal coordinates for each substructure by using Craig-Bampton transformation matrices is given as follows:

$$
[T]^{T}\left[\begin{array}{ll}
{\left[M_{L L}\right]} & {\left[M_{L R}\right]} \\
{\left[M_{R L}\right]} & {\left[M_{R R}\right]}
\end{array}\right][T]\{\ddot{q}\}+[T]^{T}\left[\begin{array}{ll}
{\left[K_{L L}\right]} & {\left[K_{L R}\right]} \\
{\left[K_{R L}\right]} & {\left[K_{R R}\right]}
\end{array}\right][T]\{q\}=[T]^{T}\left\{\begin{array}{l}
\left\{f_{L}\right\} \\
\left\{f_{R}\right\}
\end{array}\right\}
$$

Pre and post multiplication of system matrices by Craig-Bampton transformation matrix, $[T]$, uncouples the equations in modal coordinates. Resulting modal matrices of the system can be given as 


$$
\begin{gathered}
{\left[\begin{array}{cc}
{\left[\phi_{L}\right]} & {\left[\phi_{R}\right.} \\
{[0]} & {[I]}
\end{array}\right]^{T}\left[\begin{array}{cc}
{\left[K_{L L}\right]} & {\left[K_{L R}\right]} \\
{\left[K_{R L}\right]} & {\left[K_{R R}\right.}
\end{array}\right]\left[\begin{array}{cc}
{\left[\phi_{L}\right]} & {\left[\phi_{R}\right]} \\
{[0]} & {[I]}
\end{array}\right]=\left[\begin{array}{ll}
{\left[\bar{K}_{\eta \eta}\right]} & {\left[\bar{K}_{\eta R}\right]} \\
{\left[\bar{K}_{R \eta}\right]} & {\left[\bar{K}_{R R}\right]}
\end{array}\right],} \\
{\left[\begin{array}{cc}
{\left[\phi_{L}\right]} & {\left[\phi_{R}\right]} \\
{[0]} & {[I]}
\end{array}\right]^{T}\left[\begin{array}{cc}
{\left[M_{L L}\right]} & {\left[M_{L R}\right.} \\
{\left[M_{R L}\right]} & {\left[M_{R R}\right.}
\end{array}\right]\left[\begin{array}{cc}
{\left[\phi_{L}\right]} & {\left[\phi_{R}\right]} \\
{[0]} & {[I]}
\end{array}\right]=\left[\begin{array}{ll}
{\left[\bar{M}_{\eta \eta}\right]} & {\left[\bar{M}_{\eta R}\right]} \\
{\left[\bar{M}_{R \eta}\right]} & {\left[\bar{M}_{R R}\right]}
\end{array}\right],}
\end{gathered}
$$

where

$$
\begin{aligned}
& {\left[\bar{K}_{\eta \eta}\right]=\left[\omega_{S}^{2}\right],} \\
& {\left[\bar{K}_{\eta R}\right]=\left[\bar{K}_{R \eta}\right]=[0]} \\
& {\left[\bar{K}_{R R}\right]=\left[\left[K_{R R}\right]-\left[K_{R L}\right]\left[K_{L L}\right]^{-1}\left[K_{L R}\right]\right]} \\
& {\left[\bar{M}_{\eta \eta}\right]=[I]} \\
& {\left[\bar{M}_{\eta R}\right]=\left[\bar{M}_{R \eta}\right]^{T}=\left[\left[\phi_{L}\right]^{T}\left[M_{L L}\right]\left[\phi_{R}\right]+\left[\phi_{L}\right]^{T}\left[M_{L R}\right]\right]} \\
& {\left[\bar{M}_{R R}\right]=\left[\left[\phi_{R}\right]^{T}\left[M_{L L}\right]\left[\phi_{R}\right]+\left[\phi_{R}\right]^{T}\left[M_{L R}\right]+\left[M_{R L}\right]\left[\phi_{R}\right]+\left[M_{R R}\right]\right] .}
\end{aligned}
$$

Thus, reduction operation is finalized by transforming each substructure individually. If the substructures are identical as in the case of repetitive sectors of bladed disk models, the transformation matrix is needed to be calculated for once and can be applied to all substructures. This property serves as a great advantage of cyclic symmetry.

In this study, after the transformation process, coupling process takes place; however, in Craig-Bampton method, the coupling and reduction operations are separate and can be done one after the other, since the reduced and coupled degrees of freedom are distinct. If the coupling operation is done after the reduction process the equation of motion takes the form below 


$$
\begin{gathered}
{\left[\begin{array}{ccc}
{[I]} & {\left[\bar{M}_{\eta R, 1}\right]} & {[0]} \\
{\left[\bar{M}_{R \eta, 1}\right]} & {\left[\bar{M}_{R R, 1}\right]+\left[\bar{M}_{R R, 2}\right]} & {\left[\bar{M}_{R \eta, 2}\right]} \\
{[0]} & {\left[\bar{M}_{\eta R, 2}\right]} & {[I]}
\end{array}\right]\left\{\begin{array}{l}
\left\{\ddot{\eta}_{1}\right\} \\
\left.\left\{\ddot{U}_{R}\right\}\right\} \\
\left\{\ddot{\eta}_{2}\right\}
\end{array}\right\}+} \\
{\left[\begin{array}{ccc}
{\left[\omega_{s, 1}^{2}\right]} & {[0]} & {[0]} \\
{[0]} & {\left[\bar{K}_{R R, 1}\right]+\left[\bar{K}_{R R, 2}\right]} & {[0]} \\
{[0]} & {[0]} & {\left[\omega_{s, 2}^{2}\right]}
\end{array}\right]\left\{\begin{array}{l}
\left\{\eta_{1}\right\} \\
\left\{U_{R}\right\} \\
\left\{\eta_{2}\right\}
\end{array}\right\}=\left\{\begin{array}{c}
\left\{f_{\eta, 1}\right\} \\
\left\{f_{R}\right\} \\
\left\{f_{\eta, 2}\right\}
\end{array}\right\} .}
\end{gathered}
$$

In Eq. (2.8), the coupling operation of boundary degrees of freedom is exemplified on a case where only two substructures are coupled together using boundary degrees of freedom. The subscripts 1 and 2 denote the properties of first and second substructures respectively.

Finally, using Eq. (2.8) and assuming harmonic excitation, it is possible to determine the modal displacements by algebraic matrix inverse operation. In order to obtain physical displacements of the combined structure, a back transformation operation is needed. The operation is exemplified again by using the structure composed of two substructures as shown below

$$
\left\{\begin{array}{c}
\left\{U_{L, 1}\right\} \\
\left\{U_{R}\right\} \\
\left\{U_{L, 2}\right\}
\end{array}\right\}=\left[\begin{array}{ccc}
{\left[\phi_{L, 1}\right]} & {\left[\phi_{R, 1}\right]} & {[0]} \\
{[0]} & {[I]} & {[0]} \\
{[0]} & {\left[\phi_{R, 2}\right]} & {\left[\phi_{L, 2}\right]}
\end{array}\right]\left\{\begin{array}{c}
\left\{\eta_{1}\right\} \\
\left\{U_{R}\right\} \\
\left\{\eta_{2}\right\}
\end{array}\right\} .
$$

In the case of bladed disks, the substructures are either repetitions of fundamental sector or blade and disk components separately. If one of the substructures under consideration is a blade of the bladed disk structure, the transformed stiffness matrix $\left[\bar{K}_{\eta \eta}\right]$ would represent the square of the cantilevered blade natural frequencies, $\left[\Lambda_{b}\right]$. In literature, mistuning is modeled as deviations of cantilevered blade natural 
frequencies from the cantilevered blade natural frequencies of the tuned structure. The common mathematical expression of the relationship between the square of the tuned and mistuned cantilevered blade natural frequencies is given as

$$
\left[\Lambda_{b}^{\text {mist }}\right]=\left[\Lambda_{b}^{\text {tun }}\right](1+\delta)
$$

where $\left[\Lambda_{b}^{\text {mist }}\right]$ is the matrix of squares of the cantilevered blade natural frequencies of the mistuned structure, $\left[\Lambda_{b}^{\text {tun }}\right]$ is the matrix of squares of the cantilevered blade natural frequencies of the tuned structure and $\delta$ is the coefficient of mistuning. Furthermore, Bladh [25] reported the implementation of fixed based component mode synthesis method for the mistuned bladed disk finite element models using cyclic symmetry properties. In his work, Bladh concluded in system mass and stiffness matrices as shown below:

$$
\begin{gathered}
{\left[\bar{M}_{d c b}\right]=\left[\begin{array}{ccc}
{[I]} & {\left[\tilde{\mu}_{d c}\right]} & {[0]} \\
{\left[\tilde{\mu}_{d c}\right]^{T}} & {\left[\tilde{\mu}_{c c, d}\right]+[I] \otimes\left[\tilde{\mu}_{c c, b}\right]} & {[\hat{F}]^{T}\left[[I] \otimes\left[\mu_{b c}^{T}\right]\right]} \\
{[0]} & {\left[[I] \otimes\left[\mu_{b c}\right]\right][\hat{F}]} & {[I]}
\end{array}\right],} \\
{\left[\bar{K}_{d c b}\right]=\left[\begin{array}{ccc}
{\left[\tilde{\Lambda}_{d}\right]} & {[0]} & {[0]} \\
{[0]} & {\left[\tilde{\kappa}_{c c, d}\right]+[I] \otimes\left[\tilde{\kappa}_{c c, b}\right]} & {[0]} \\
{[0]} & {[0]} & {[I] \otimes\left[\Lambda_{b}^{\text {mist }}\right]}
\end{array}\right] .}
\end{gathered}
$$

In Eq. (2.11), $\left[\bar{K}_{d c b}\right]$ and $\left[\bar{M}_{d c b}\right]$ represent reduced mass and stiffness matrices respectively. Tilde scripts over the parameters represent that the parameter is defined in cyclic coordinates. $[\hat{F}]$ is the Fourier transformation matrix from cyclic coordinates to physical coordinates. $\left[\tilde{\mu}_{d c}\right]$ and $\left[\mu_{b c}\right]$ are the transformed fixed-base disk and blade mass matrices; $\left[\tilde{\Lambda}_{d}\right]$ and $\left[\Lambda_{b}\right]$ are the transformed fixed-base disk and 
mistuned blade stiffness matrices; respectively. The presented formulation of Bladh is used in this thesis for determining physical displacements of bladed disk assembly nodes by reflecting mistuning to each individual blade mathematically as shown below:

$$
\left[\Lambda_{b}^{\text {mist }}\right]=\mathbf{B d i a g}_{n=1 \ldots N}\left[\operatorname{diag}_{k=1 \ldots m_{b}}\left(\left[1+\delta_{n}^{k}\right]\left[\Lambda_{b}^{\text {tun }}\right]\right)\right]
$$

where $N$ is the number of blades, $m_{b}$ is the number of cantilevered blade modes used after the truncation process. 


\section{CHAPTER 3}

\section{UNCERTAINTY QUANTIFICATION IN MISTUNING}

\subsection{Uncertainty Types}

Every engineering system has its own uncertainties occurring from the system itself or from the operating medium. In general, types of uncertainties related to engineering phenomena are investigated under the following four groups; randomness, vagueness, ambiguity and imprecision [26]. Randomness is a type of uncertainty which is defined for the situations where the outcome of an experiment or event can be measured but the result of the event cannot be determined before an experiment is conducted. For a random uncertainty, the character of the uncertainty is determined by conducting enough number of experiments and ranking the number of occurrences of the outputs. Therefore, the randomness is related to the quantity of the uncertain information. However, the other three types of uncertainty definitions are related to the quality or the meaning of the uncertain information. Ambiguity occurs when the information about the uncertainty is gathered but the meaning of the information cannot be interpreted clearly. Vagueness represents a situation where the meaning of the information might mean more than one meanings and imprecision occurs where there exists lack of information. Regarding mistuning studies two of the uncertainties mentioned, random uncertainties and imprecise uncertainties are taken into account in this thesis.

One of the basic causes of mistuning is deviation occurring due to manufacturing tolerances. The deviation of geometry and material properties destroy cyclic symmetry and cause mistuning in bladed disk systems. In this situation, the value of deviation due to tolerances is measurable but it cannot be determined before an 
experiment is conducted. This fact leads the uncertainty due to manufacturing tolerances to be a random uncertainty. On the contrary, there are some other causes of mistuning that even cannot be measured precisely. For instance, another important sources of mistuning, i.e. root stiffness and damping parameters, are function of boundary conditions and change depending on the state of the bladed disk. In this situation, the only information regarding the deviation of root stiffness or damping values would be the extent of deviation of the parameter for a given bladed disk; for instance the deviation might be $5 \%$ at most. This type of uncertainty in mistuning is a class of imprecise uncertainty.

Independent from the cause of mistuning, the aim of mistuning studies is to assign a reliability value to blade forced response values for the reliability analysis and determine the worst-case situation that is possible to occur within the uncertainty limits. This requires correct management of uncertainties in mistuned bladed disk systems. In literature, there are two methods proposed to handle uncertainties in engineering systems: probabilistic methods and possibilistic methods.

\subsection{Uncertainty Management Methods}

Compatible with their names, probabilistic methods are based on the theory of probability and possibilistic methods are based on the theory of possibility. Probabilistic methods are proposed for handling random uncertainties and possibilistic methods are used for handling imprecise uncertainties. These methods utilize probability distributions and possibility distributions to characterize the random and imprecise uncertainties, respectively, and the possibility/probability consistency theorem represents the connection between the two types of characterizations.

In probabilistic methods, basically, expected values of outcomes of an uncertain situation are interpreted by processing relative frequency of outputs which are obtained by processing random sets of inputs. In mistuning studies, random sets 
containing values of uncertain mistuning variables are used in Monte-Carlo analysis in order to come-up with random sets of values of mistuned blade forced responses. Monte-Carlo analysis picks one value from each random set of uncertain mistuning variables at every iteration, and especially for mistuning studies, the set of picked values establishes a mistuning pattern. The mistuning pattern for the current iteration results in corresponding mistuned forced response values. The process of pursuing iterations for a number of times creates a random set of outputs. The probability density functions of calculated (random) mistuned blade forced response values are used for the purpose of reliability analysis. Moreover, the worst-case forced response value is determined to be the maximum value present in the random data set of mistuned forced responses.

For the study of possibilistic interpretation of mistuning, information regarding uncertain mistuning parameters is assumed to be imprecise. Correspondingly, in order to come up with a reliable solution to imprecise forced response parameters in a mistuned structure, fuzzy set theory and possibility theory are utilized together. Regarding to the possibility theory, imprecise variables are modeled as fuzzy variables possessing possibility distributions and required mathematical operations regarding fuzzy variables are governed by the fuzzy arithmetic, based on the rules of fuzzy set theory [27], all developed by A. Lutfi Zadeh.

The proposition $p$ : 'deviation in stiffness parameters of structure $\mathrm{Y}$ is $\pm 5 \%$ at most' represents an example to the imprecise information of a mistuning parameter. The proposition carries fuzziness on the values that are going to be assigned to the imprecise variable, deviation in stiffness parameters of structure $\mathrm{Y}, D_{k}$. Due to the fuzziness in meaning of the proposition $p$, the imprecise parameter $D_{k}$ is modeled as fuzzy variable possessing possibility distribution $\pi_{k}$. In order to be able to explain the meaning of the concept of possibility distribution, let us suppose that the universe of discourse $M$ with a generic element $m$, carries all possible values of any stiffness value. Then, the proposition ' $\pm 5 \%$ at most' defines a unique fuzzy subset $F$ which is characterized by the membership function $\mu_{F}$, governing the compatibility of $m$ with the proposition. Namely, regarding to the definition of fuzzy sets [27], every value $m$ 
takes a membership function value $\mu_{F}(m)$ that ranges from 0 to 1 denoting the degree of membership of $m$ to the fuzzy set $F$ Therefore, the element $m$ within the universe of discourse $M$ which does not belong to the fuzzy set $F$ takes a membership value of zero. Furthermore, the grade of membership of $m$ in $F$ increases as the membership function value $\mu_{F}(m)$ gets closer to unity.

According to the definition of the concept of possibility distribution [13], the proposition $p$ induces a possibility distribution $\pi_{k}$ which equates the possibility of $D_{k}$ taking value $m$ to $\mu_{F}(m)$. In this way $D_{k}$ becomes a fuzzy variable which is associated with the possibility distribution $\pi_{k}$. For instance, let us suppose that the value of the stiffness parameters are designed to be $4 \mathrm{kN} / \mathrm{m}$. Regarding the proposition $p$, stiffness parameters can take values between 3.8 and $4.2 \mathrm{kN} / \mathrm{m}$. Then in order to represent the possibility distribution $\pi_{k}$ of fuzzy variable $D_{k}$, a triangular fuzzy number $\bar{A}(3.8 / 4 / 4.2)$ can be used as shown in Figure 3.1;

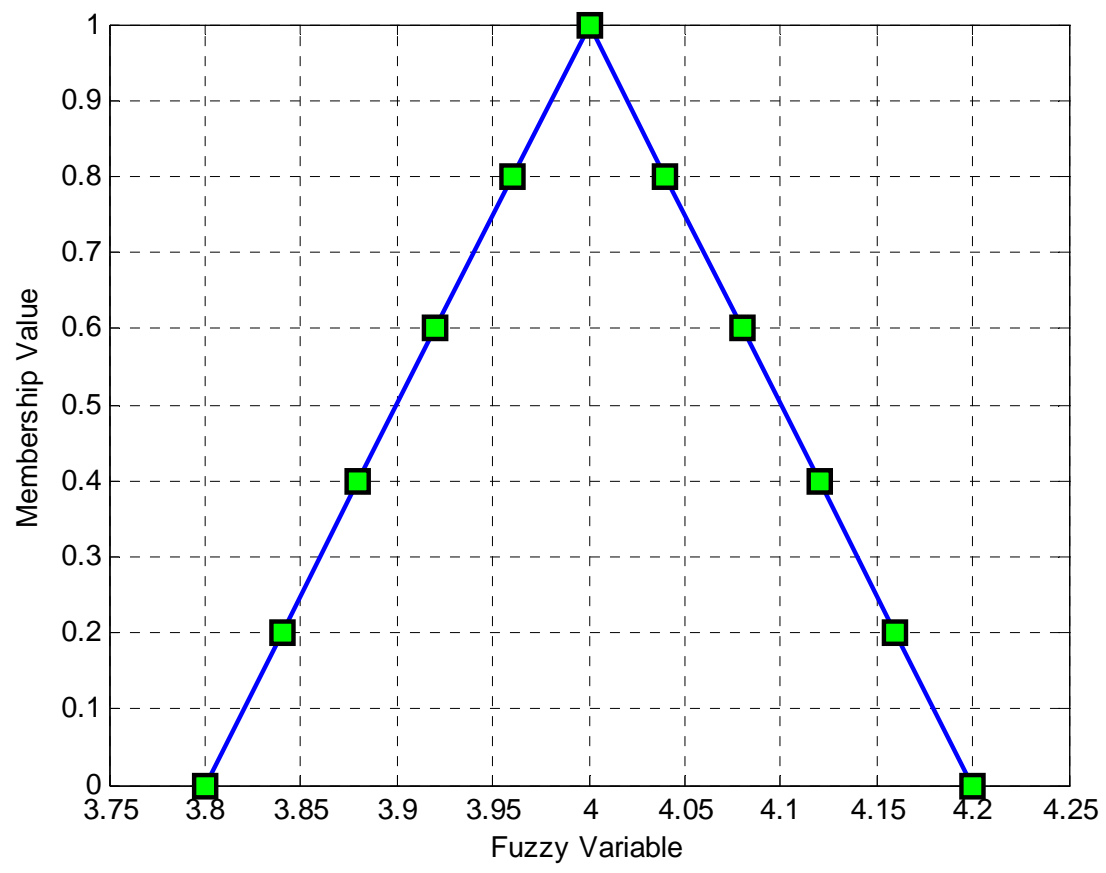

Figure 3.1: A triangular membership function distribution 
where for a triangular fuzzy number $\bar{A}(x / y / z)$, the three numbers $x<y<z$ identifies the base of the triangular possibility distribution by the interval $[x, z]$ and its vertex by $y$ [20]. Figure 3.1 states that the values of the stiffness parameters are designed to be $4 \mathrm{kN} / \mathrm{m}$ and they are not expected to be more than 4.2 or less than 3.8 $\mathrm{kN} / \mathrm{m}$. Furthermore, it is observed that regarding proposition $p$, assessor is more confident to assign $3.9 \mathrm{kN} / \mathrm{m}$ to the stiffness value than $4.15 \mathrm{kN} / \mathrm{m}$ since $3.9 \mathrm{kN} / \mathrm{m}$ has larger possibility value of $\sim 0.5$ compared to $\sim 0.25$ for $4.15 \mathrm{kN} / \mathrm{m}$. In possibilistic analysis, range of possible values of outcomes are sought and organized for each and every confidence level.

The connection between possibilistic and probabilistic methods are established upon the logical truth stating that 'probability of a measured event cannot be more than the possibility of that event' [1] which is based on possibility/probability consistency theorem proposed by Zadeh [13]. Due to the nature of the theorem, interpretation of the connection remains open to variations according to situation at hand. The interpretation of consistency principle in mistuning studies is detailed in second case study where the performances of the two methods are compared on the success of reliability analysis and on determining the worst-case response. 


\section{CHAPTER 4}

\section{METHODOLOGY}

In this thesis, within the context of possibilistic interpretation of mistuning, ways of determining limits of possible mistuned blade forced responses under the effect of possible imprecise mistuning parameters in each and every confidence level are investigated. This chapter is devoted to mathematics and methodology of the possibilistic interpretation of mistuning.

\subsection{Fuzzy Algebra}

It is known from previous section that imprecise uncertainties are mathematically modeled as fuzzy variables. Presence of a fuzzy variable within an equation transforms the crisp equation into a fuzzy equation; therefore for possibilistic interpretation of mistuning, in order to be able to determine fuzzy forced vibration response, the governing fuzzy dynamic equation must be solved. There exist two methods for the solution of fuzzy equations: extension principle solution and interval arithmetic solution.

The methods used for the solution of fuzzy equations originate from the solution of basic algebraic equations. For two fuzzy numbers $\bar{A}$ and $\bar{B}$, if $\bar{A} * \bar{B}=\bar{C}$, $*$ denoting addition, multiplication, subtraction or division, then extension principle defines membership function for $\bar{C}$ as; 


$$
\bar{C}(z)=\max _{x, y}\{\min (\bar{A}(x), \bar{B}(y)) \mid x * y=z\}
$$

Regarding Eq. (4.1), in order to determine membership function for $\bar{C}$, extension principle solution searches for all $[x, y]_{i}$ pairs that constitute $z_{i}$ and records minimum membership function value within the pairs $\min \left(\bar{A}\left(x_{i}\right), \bar{B}\left(y_{i}\right)\right)$ as $\bar{C}\left(z_{i}\right)$. Then, the record that has the maximum membership function value $\max \left(\bar{C}\left(z_{i}\right)\right)$ is finally assigned as the membership function value for $\bar{C}(z)$.

In contrast, interval arithmetic method uses alpha-cuts to represent the membership function distribution of each fuzzy variable and utilizes interval arithmetic methods for the solution of basic algebraic equations. Alpha-cuts are slices of possibility distributions producing confidence levels as non-fuzzy-crisp intervals, as shown in Figure 3.1 by squared marks. For the closed-bounded intervals of alpha-cuts of two fuzzy numbers $\bar{A}$ and $\bar{B}, \bar{A}[\alpha]=\left[a_{1}(\alpha), a_{2}(\alpha)\right]$ and $\bar{B}[\alpha]=\left[b_{1}(\alpha), b_{2}(\alpha)\right]$ (for instance, the least confidence level of fuzzy variable in Figure 3.1 can be written as $\bar{A}[0]=[3.8,4.2])$, interval arithmetic solution determines the membership function distribution by using interval arithmetic rules at each and every confidence level [28]:

$$
\bar{A}[\alpha] * \bar{B}[\alpha]=\bar{C}[\alpha]
$$

It is known that as interval arithmetic methods are used in solution of engineering problems, the rules of interval arithmetic conflict with the physical structure integrity and creates dependency problem [28]. In contrast, extension principle solution can be adapted to alpha-cut methodology [20]; hence, approximates the true solution practically and without creating dependency issue. Therefore, for the solutions of fuzzy equations extension principle solution is used in this study. The details of the solution are explained under the next topic. 


\subsection{Solutions of Fuzzy Equations}

Mathematically speaking, fuzzy functions are mapping from fuzzy numbers to fuzzy numbers. They are usually extended from real valued functions. According to extension principle [20], a real-valued function $h:[x, y] \rightarrow z$ can be extended to the corresponding fuzzy function $\bar{H}(\bar{X}, \bar{Y})=\bar{Z}$ as follows:

$$
\bar{Z}(z)=\max _{x, y}\left\{\min (\bar{X}(x), \bar{Y}(y)) \mid h(x, y)=z, a_{1} \leq x \leq b_{1}, a_{2} \leq x \leq b_{2}\right\}
$$

According to Eq. (4.3), in order to be able to assess the membership function value of the dependent variable $\bar{Z}(z)$, first, all pairs of the independent variables $(x, y)$ that are mapped into $z$ should be determined regardless of their confidence levels. This makes use of extension principle impossible on computer, since it is impossible to determine all the pairs for all types of equations. However, Buckley et. al. [20] proposes that, if the real-valued function $h$ is continuous, then upper and lower bounds of the alpha-cuts of fuzzy function $\bar{Z}[\alpha]=\left[z_{1}(\alpha), z_{2}(\alpha)\right]$ can be determined as follows:

$$
\begin{gathered}
z_{1}(\alpha)=\min \{h(x, y) \mid x \in \bar{X}[\alpha], y \in \bar{Y}[\alpha]\} \\
z_{2}(\alpha)=\max \{h(x, y) \mid x \in \bar{X}[\alpha], y \in \bar{Y}[\alpha]\} \\
0 \leq \alpha \leq 1
\end{gathered}
$$

where the $\min / \max$ operations are to be carried out by optimization algorithms. Eq. (4.4) uses alpha-cut representation of possibility distributions. For instance, the fuzzy variable in Figure 3.1 can be expressed in terms of its alpha-cuts as $\bar{A}[\alpha]=$ $[3.8+0.2 \alpha, 4.2-0.2 \alpha]$, where $0 \leq \alpha \leq 1$. Therefore, instead of declaring the 
shape of the possibility distribution as $\bar{A}(x / y / z)$, the values that share the same confidence level is declared as intervals to represent the fuzzy variable.

By using alpha-cut representation of fuzzy variables in solutions of fuzzy equations, Eq. (4.4) approximates the true solution by utilizing intervals of independent variables from the same confidence level. Namely, instead of searching all possible independent variable pairs from any confidence level, the ones that share the same

possibility distribution value $\bar{X}[\alpha]$ and $\bar{Y}[\alpha]$ are used to determine the interval of dependent variables with the same possibility distribution value, $\bar{Z}[\alpha]$. This approximation makes the use of extension principle possible on computer; hence, establishes the basis for the solution of fuzzy functions and equations.

\subsection{Fuzzy Forced Response Analysis}

In mistuning studies, the solution of fuzzy dynamic equation is sought in order to come up with fuzzy forced response values. In mathematical terms, fuzzy dynamic equation is an extension of real valued dynamic equation; therefore, in this case the real valued function $h$ defined in previous section represents the real valued forced response problem and the extended fuzzy function $H$ corresponds to the fuzzy forced response problem.

For a crisp (non-fuzzy) lumped-parameter system with structural damping, the crisp equation of motion is given as follows:

$$
[M]\{\ddot{q}\}+\left[K^{*}\right]\{q\}=\{F\},
$$

where $\left[K^{*}\right]$ is the complex stiffness matrix including structural damping, $[M]$ is the mass matrix, $\{F\}$ is the excitation force vector, $\{q\}$ and $\{\ddot{q}\}$ represent displacement 
and acceleration vectors, respectively. The crisp equation of motion given in Eq. (4.5) can be solved by modal analysis.

If the system elements are considered as imprecise so as fuzzy variables, the equation of motion transforms into the fuzzy equation of motion:

$$
[\bar{M}]\{\ddot{\bar{q}}\}+\left[\bar{K}^{*}\right]\{\bar{q}\}=\{\bar{F}\} .
$$

where over-bar expresses fuzzy matrices and vectors. Then, as stated in previous section, for the solution of fuzzy equation of motion the corresponding real valued equation of motion needs to be continuous. Since mass and stiffness matrices reflect linear physical systems, the solution to Eq. (4.5) needs to be continuous. Therefore, the equation that is used for determining alpha-cuts of the solution, Eq. (4.4), is valid. Correspondingly the algorithm $\Phi$ would accept alpha-cuts of elements fuzzy correspondence of the input matrices and vector, $[\bar{M}],\left[\bar{K}^{*}\right],\{\bar{F}\}$, such as $\bar{m}_{i j}[\alpha]$, $\bar{k}_{i j}[\alpha], \bar{f}_{i}[\alpha]$ and would return the alpha-cuts of elements of fuzzy displacement vector $\{\bar{q}\}, \bar{q}_{i}[\alpha]$ as the output. Then, according to Eq. (4.4), assuming the excitation force vector is exactly known, i.e. crisp, alpha-cuts of the blade displacement forced response values with respect to deviations in system parameters are calculated as follows:

$$
\begin{gathered}
\bar{q}[\alpha]=\left[q_{1}(\alpha), q_{2}(\alpha)\right], \\
q_{1}(\alpha)=\min \left\{\Phi\left(k_{11}, \ldots, k_{n n}, m_{11}, \ldots, m_{n n}\right) \mid k_{i j} \in \bar{k}_{i j}[\alpha], m_{i j} \in \bar{m}_{i j}[\alpha]\right\} \\
q_{2}(\alpha)=\max \left\{\Phi\left(k_{11}, \ldots, k_{n n}, m_{11}, \ldots, m_{n n}\right) \mid k_{i j} \in \bar{k}_{i j}[\alpha], m_{i j} \in \bar{m}_{i j}[\alpha]\right\}
\end{gathered}
$$

Consequently, for a vibrating system with imprecise parameters regarding the proposition 'deviation in stiffness parameters of structure is $\pm 5 \%$ at most' can be 
solved for alpha-cuts of the blade fuzzy forced response elements practically and without creating dependency issue using Eq. (4.7). For the min/max operations in Eq. (4.7) several optimization techniques can be used. The uses of genetic algorithm as global optimization method and constraint non-linear minimization algorithm as gradient-based algorithm in the solution of fuzzy equation of motion are detailed in the next section.

\subsection{Use of Optimization Methods on Solutions to Fuzzy Dynamic Equations}

\subsubsection{Global Optimization Methods}

Genetic algorithm is a type of evolutionary global optimization tool that generate solutions using techniques inspired by natural evolution such as inheritance, mutation, selection and crossover. Global optimization algorithms are utilized for the problems where the global minimum or maximum are sought. However, these algorithms are based on random processes; therefore, they conclude in different results at each run. Genetic algorithm is utilized in order to find the bounds of fuzzy blade displacement magnitudes in Eq. (4.7) and effects of utilizing several options of genetic algorithm are investigated separately.

\subsubsection{Gradient-Based Optimization Algorithm}

Constraint minimization algorithm is a type of gradient-based optimization method. Gradient-based optimization methods are fast algorithms that converge to the solution using gradients of the objective function; however, they require 'good' initial guesses, if not, they might diverge easily or converge to a local optimum point. In mistuning studies sensitivity coefficients are utilized in order to be able to use gradient based algorithms; however, in this thesis gradient based algorithms are utilized without using sensitivity coefficients. An effective gradient based algorithm 
is proposed using the solutions at the previous alpha-cuts as the initial guess for the next alpha-cut iteration. The computational and accuracy-wise effectiveness of this method is compared with the efficiency of the hybrid optimization method of determining the worst-case solutions utilizing the least computational time.

The efficiency of the method comes from the fact that in each level of confidence the interval of the uncertain parameter values capsulates the interval of the uncertain parameters in previous levels of confidence. Hence, it is logical to expect at least as much worse case as the one previous solution. This fact creates continuity between the solutions of levels of confidences and initial guess determination processes of gradient-based algorithms based on the continuity property of the solutions. Next section explains different interpretations of next level of confidence and initial guess determination processes.

\subsubsection{Types of Next Level of Confidence and Initial Guess Determination Process}

It is possible to use solutions of previous level of confidences in order to make an initial guess for the solution of present level of confidence. In its most basic sense, the solution of previous level of confidence is used as the initial guess for the present level of confidence. The other ways of determining initial guess values for the present level of confidence makes logical combinations out of previous levels of confidence solutions. If a polynomial is fit to solutions of previous levels of confidences then the initial guess value for the present level of confidence can be determined easily by using the analytical formula of the polynomial fit.

The aim in the worst-case determination studies is to determine the worst-case situation in least computational time; therefore, the next level of confidence should be selected appropriate to the complexity of the system at hand. The closer initial guess values are to the solution of the level of confidence, the faster the gradientbased method is. On the other hand, using too many levels of confidence would 
increase the computational time; consequently, an optimization strategy is required for the next level of confidence determination problem. The next level of confidence can either be determined by studying how close are the initial guesses to the values of the uncertain parameters that result in the worst-case situation in the previous levels of confidence or by checking the number of iterations done at the previous levels of confidence. Both of these ways are investigated in this study. 


\section{CHAPTER 5}

\section{CASE STUDIES}

In this chapter, three numerical cases are given for exemplifying the concept of possibilistic interpretation of mistuning numerically, for comparing reliability and computational efficiency performances of possibilistic methods with respect to probabilistic methods, and for showing the benefits of using the proposed novel possibilistic worst-case determination algorithm in mistuning studies. The first case study seeks for the worst-possible effects of mistuning on a cyclically symmetric lumped parameter bladed disk system for low, moderate and high risk levels of intervals. In the second case study, the meaning of the worst-possible situations are further explained by investigating differences between the worst-possible situations and the most-probable worst-case situations using the results found in the first case study. This case study also compares the computational time required and accuracy of both methods in determining the worst-case that is possible to occur for a mistuned bladed disk under engine order excitation. The last case study uses a realistic reduced order finite element model of a bladed disk in order to investigate performances of current optimization based methods and the proposed novel possibilistic method in determining the worst-case response.

\subsection{CASE I - Possibilistic Interpretation of Mistuning}

Numerical example considered in this section is designed to exemplify the concept of possibilistic interpretation of mistuning on a bladed disk system by determining deviation of the worst-possible effects of mistuning between low, moderate and high 
risk levels. In this case study, a mistuned bladed disk system is assumed to be operating on a static pressure field. The static pressure field applies harmonic forces to the blades that are constant in magnitude but differs in phase among the blades called engine order forcing. For the present case, mistuning is modeled to be affecting only stiffness parameters of the bladed disk system. The only information regarding the effect of mistuning is that the deviation of stiffness parameters can be at most $\pm 5 \%$. Within given deviation range, the worst case response is sought for defined possible engine order types (orders 1, 2, 3,4) and for identified range of excitation frequency. For the determined worst-case scenario, deviation of the worstcase response for low, moderate and high risk levels are investigated.

The worst-case scenario for mistuning considerations is the case where the energy is localized on a particular blade. Since energy localization results in much higher displacement values on that particular blade compared to the rest of the blades, the maximum possible blade displacements for each engine order excitation is sought and compared with the rest of the blades in order to determine the worst-case situation where the energy localization occurs.

Energy localization results from destruction of cyclic symmetry due to uncertainties like manufacturing tolerances, wear in service and material non-uniformities. In this study, these uncertainties are treated as imprecise and assumed to be affecting stiffness parameters only. The stiffness parameters are expected to deviate at most $\pm 5 \%$ from the tuned case stiffness parameters. $\pm 5 \%$ deviation in imprecise parameter is named as $\pm 5 \%$ mistuning level in this study.

The assumption regarding imprecision in system parameters requires fuzzy modeling of stiffness parameters and the use of possibilistic analysis in order to determine maximum possible blade displacement value. Therefore, possibility distributions of fuzzy variables are determined and used in fuzzy forced response analysis using extension principle solution that utilizes alpha-cut methodology and genetic algorithm as stated in Eq. (4.7) with only fuzzy stiffness parameters $\bar{k}_{d}[\alpha], \bar{k}_{b}[\alpha]$. 
Let us suppose that elements of bladed disk model are realized with non-fuzzy, crisp values given in Table 5.1 .

Table 5.1 : Physical values of the lumped parameter model

\begin{tabular}{|c|c|}
\hline Parameter & Value \\
\hline Disk Mass, $m_{d}$ & $4 \mathrm{~kg}$ \\
\hline Disk Stiffness, $k_{d}$ & $60000 \mathrm{~N} / \mathrm{m}$ \\
\hline Blade Mass, $m_{b}$ & $2 \mathrm{~kg}$ \\
\hline Blade Stiffness, $k_{d}$ & $7000 \mathrm{~N} / \mathrm{m}$ \\
\hline Structural Damping Ratio, $\gamma$ & 0.01 \\
\hline
\end{tabular}

All system parameters except stiffness parameters are used in possibilistic analysis with the tuned case values given in Table 5.1 and stiffness parameters are modeled as triangular fuzzy variables. A triangular possibility distribution has three components, i.e. two base values and a vertex value. For fuzzy stiffness values, tuned case value given is assigned to the vertex of the corresponding fuzzy variable and base values are chosen to be $\pm 5 \%$ deviated from the tuned case value compatible with the assumption, as shown in Figure 5.1

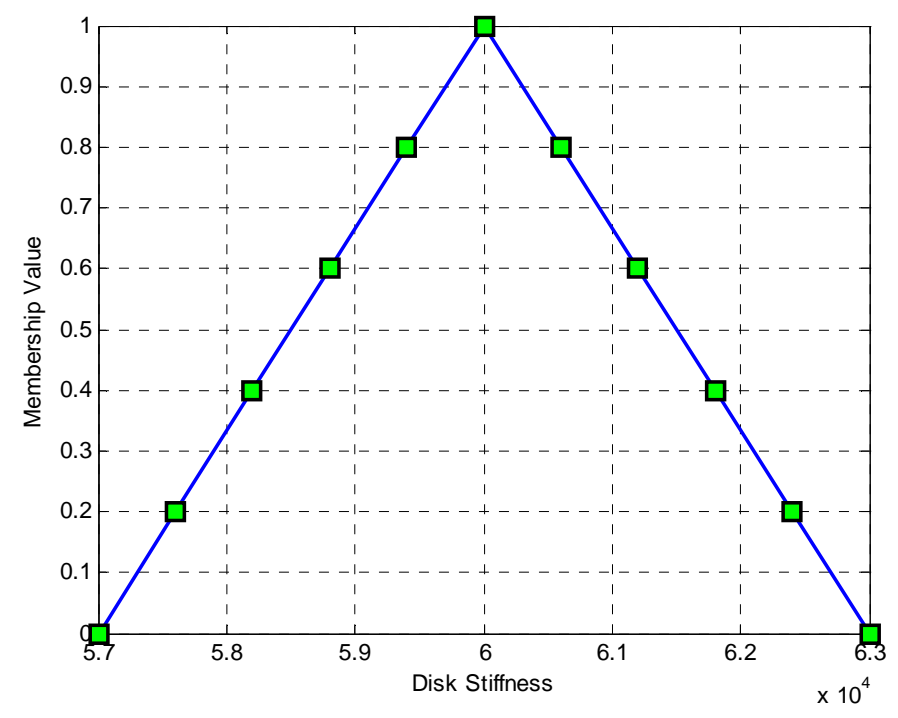

Figure 5.1 : Possibility distribution for disk stiffness parameters 
Furthermore, for the possibility distribution given in Figure 5.1 assessments of low, moderate and high risk levels are shown in Figure 5.2.

The engine order excitation which can be modeled as [22]:

where , , and are the blade number, engine order, number of blades, rotational speed and excitation amplitude, respectively. Excitation amplitude of $1 \mathrm{~N}$ is used in this study. Within the frequency range of interest the worst-possible case is determined for at most $\pm 5 \%$ mistuning level and the deviation of mistuning effects among low, moderate and high risk levels are determined.

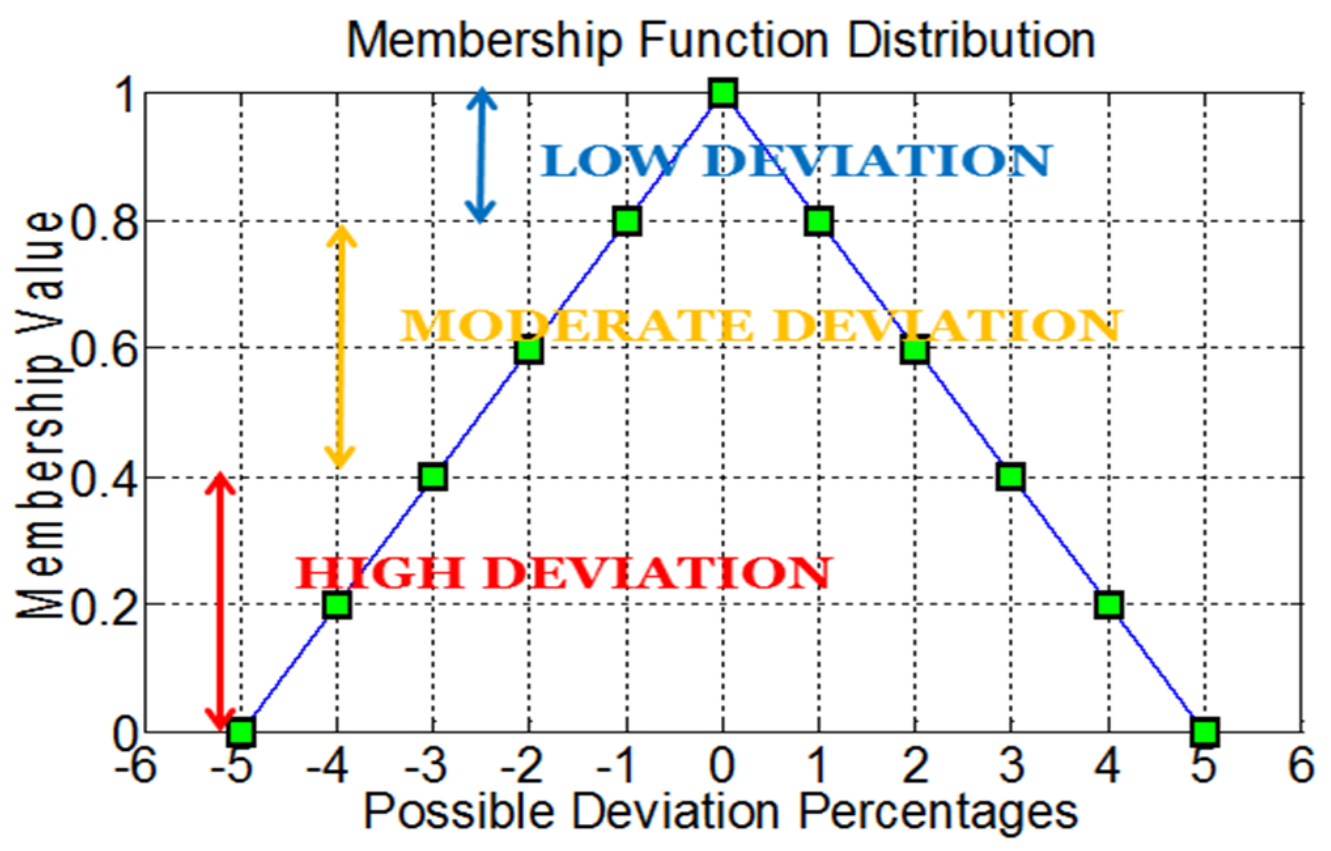

Figure 5.2: Low, moderate and high risk level assessments 


\subsubsection{Numerical Results}

The effects of engine orders 1, 2, 3 and 4 of $1 \mathrm{~N}$ forcing amplitude are sought for the worst-case analysis. In order to determine the mode that each type of excitation excites, the frequency term $m \Omega$ is swept within the frequency range of interest. For 0.9 confidence level (corresponding to $\pm 0.5 \%$ mistuning level), at each forcing frequency, the displacement of the blade corresponding to the maximum response is given for different engine order excitations in Figure 5.3.

From Figure 5.3 it can clearly be seen that different engine order excitations excite different modes of vibration at different resonant frequencies. Also, the figure highlights the maximum possible blade displacements recorded within the bladed disk for a forcing of $1 \mathrm{~N}$ amplitude and $\pm 0.5 \%$ mistuning level within the forcing frequency range of interest.

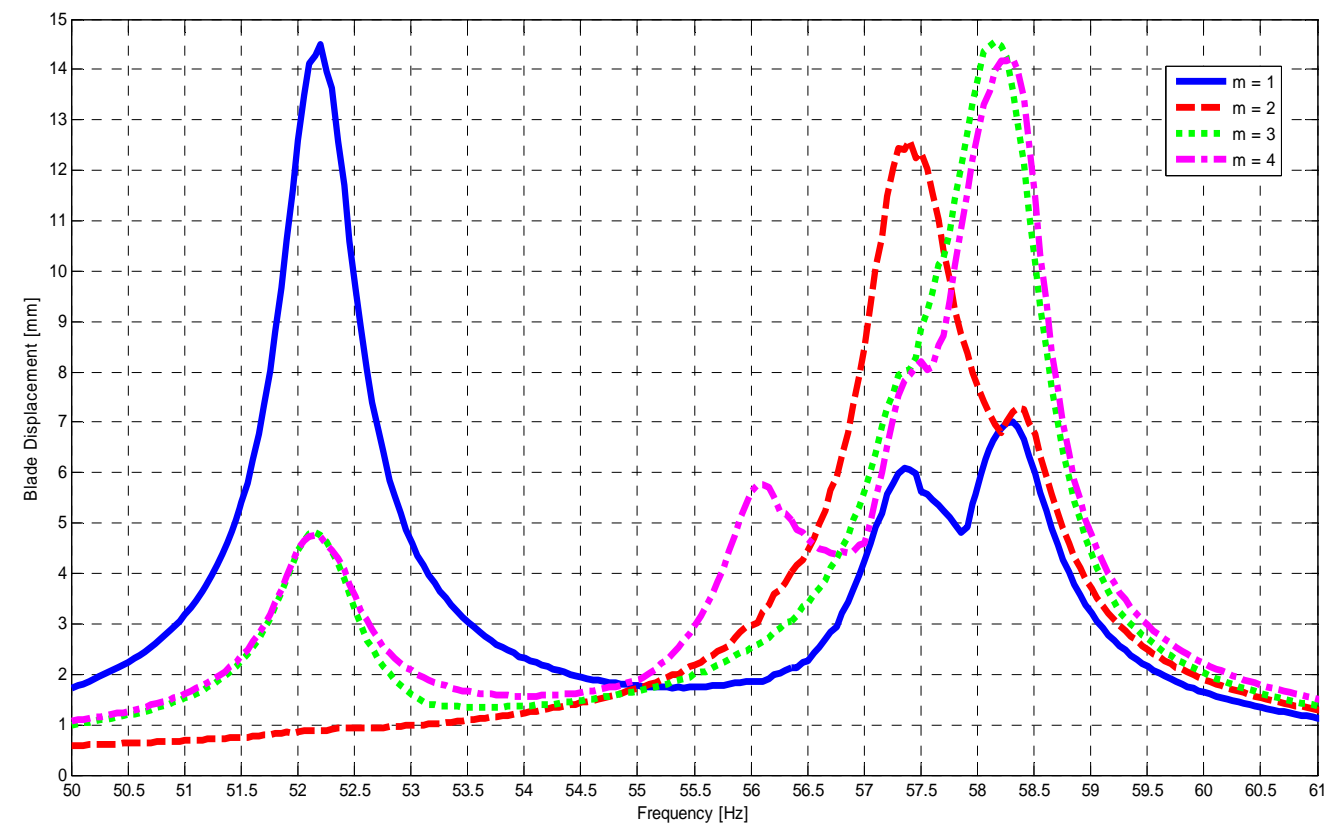

Figure 5.3 : Displacement of the blade corresponding to the maximum response vs. frequency for engine orders 1, 2, 3 and 4 ( $\pm 0.5 \%$ mistuning level) 
After frequency sweep the resonant excitation frequency values for each engine order type is determined. In order to determine the worst-possible case for each engine order excitation, the bladed disk system is assumed to be operating under the excitation of corresponding frequency for each engine order excitation type and the worst blade forced response values are sought at $\pm 5 \%$ mistuning level. In order to observe the effect of mistuning, together with the blade that has the maximum displacement, the vibration amplitudes of rest of the blades are given in Figure 5.4 as bar plots for four different engine orders at $\pm 5 \%$ mistuning level.
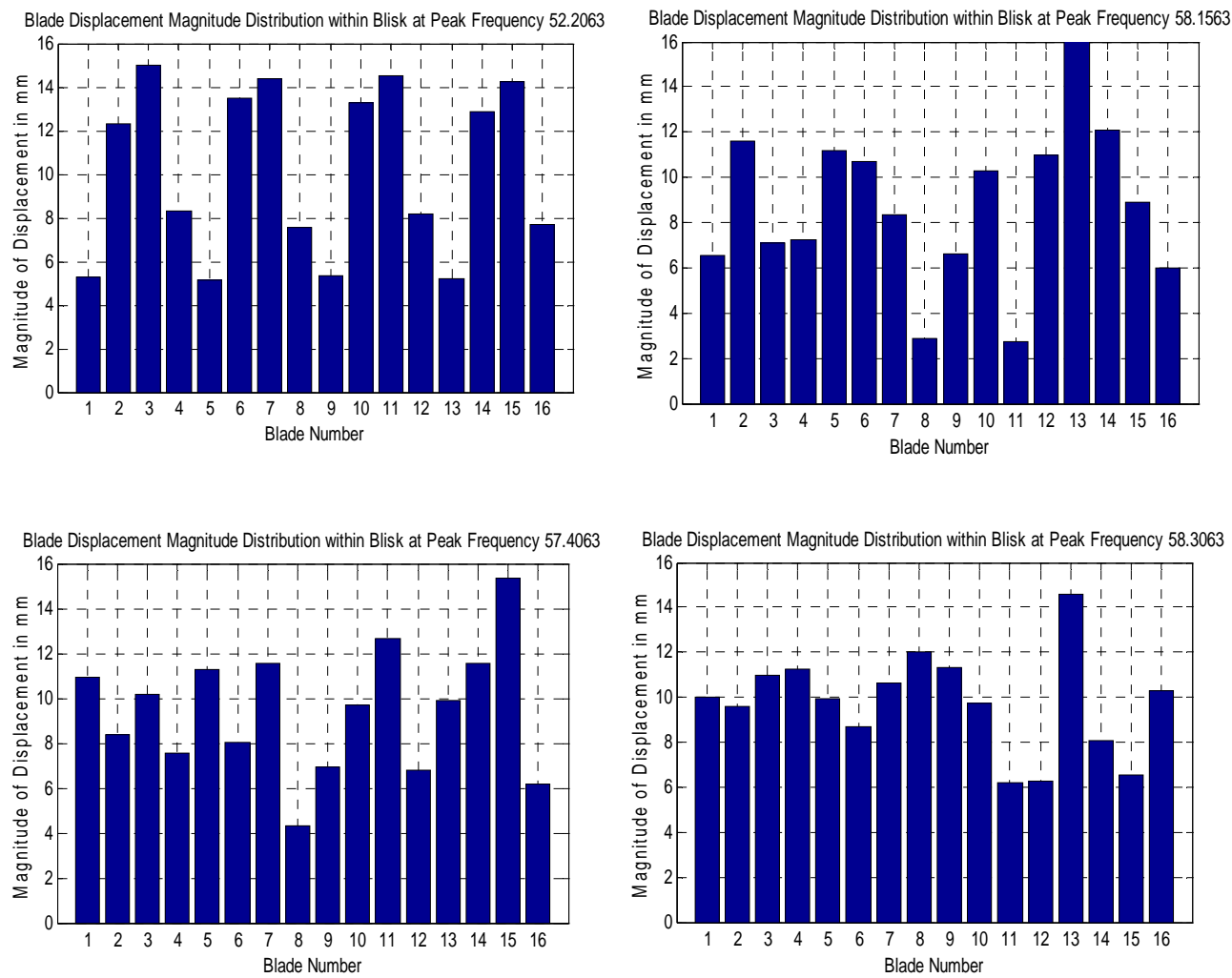

Figure 5.4 : Maximum response of blades for engine orders 1, 2, 3 and 4 ( $\pm 5 \%$ mistuning level) 
From Figure 5.4, among engine orders 1, 2, 3 and 4, the energy localization for engine orders 3 and 4 can clearly be seen in the figure where a single blade has a much larger response compared to all other blades. Moreover, engine order 3 shows the highest maximum blade forced response compared to other cases. Therefore, the worst-possible case is determined to be the case where the blisk is excited by engine order 3 for $\pm 5 \%$ mistuning level.

Finally, the deviation of worst-case results among the low, moderate and high risk levels are investigated. Table 5.2 gives the highest percent amplification factors on several levels of confidence levels and corresponding highest percent amplification factor values in low, moderate and high risk levels are tabulated in Table 5.3.

Table 5.2 : Deviation of Mistuning Effects among Different Levels of Confidence

\begin{tabular}{|c|c|c|c|c|}
\hline $\begin{array}{c}\text { Level of } \\
\text { Confidence }\end{array}$ & $\begin{array}{c}\text { Mistuning } \\
\text { Level }\end{array}$ & $\begin{array}{c}\text { Maximum Possible } \\
\text { Blade Displacement } \\
{[\mathrm{mm}]}\end{array}$ & $\begin{array}{c}\text { Maximum } \\
\text { Percent } \\
\text { Amplification }\end{array}$ & $\begin{array}{c}\text { Excitation } \\
\text { Frequency } \\
{[\mathrm{Hz}]}\end{array}$ \\
\hline 1 & 0 & 15 & - & 58.10 \\
\hline 0.8 & $\pm 1 \%$ & 16 & $7 \%$ & 58.17 \\
\hline 0.6 & $\pm 2 \%$ & 17 & $13 \%$ & 57.95 \\
\hline 0.4 & $\pm 3 \%$ & 18 & $27 \%$ & 58.2 \\
\hline 0.2 & $\pm 4 \%$ & 19 & $33 \%$ & 57.6 \\
\hline 0 & $\pm 5 \%$ & 20 & 57 \\
\hline
\end{tabular}

Table 5.3 : Deviation of Mistuning Effects among the Risk Levels

\begin{tabular}{|c|c|c|c|}
\hline $\begin{array}{c}\text { Risk } \\
\text { Level }\end{array}$ & $\begin{array}{c}\text { Maximum Possible Blade } \\
\text { Displacement }[\mathrm{mm}]\end{array}$ & $\begin{array}{c}\text { Maximum Percent } \\
\text { Amplification }\end{array}$ & $\begin{array}{c}\text { Excitation } \\
\text { Frequency }[\mathrm{Hz}]\end{array}$ \\
\hline Low & 16 & $7 \%$ & 58.17 \\
\hline Moderate & 18 & $20 \%$ & 58.20 \\
\hline High & 20 & $33 \%$ & 57.00 \\
\hline
\end{tabular}


Figures of percent amplification in Table 5.2 and Table 5.3 show the effect of increase in mistuning on forced responses in terms of percent amplifications.

\subsection{CASE II - Comparison between Uncertainty Management Methods}

In this study, methods that characterize the same uncertain situation in two different ways are compared with each other on the basis of reliability and computational efficiency in determining the worst-case situations in bladed disk assemblies. Probabilistic methods model uncertain variables as random variables and characterize uncertainties by probability density functions. In this method, expected values of outcomes of uncertain situation are interpreted by processing relative frequency of outputs obtained for the input random set. On the other hand, in possibilistic methods, uncertain parameters are modeled as fuzzy variables and characterized by possibility distributions [27].

The connection between possibilistic and probabilistic methods are established upon the logical truth stating that 'probability of a measured event cannot be more than possibility of that event' [1] which is based on possibility/probability consistency theorem proposed by Zadeh [13]. Due to nature of the theorem, interpretation of the connection remains open to variations according to situation at hand. In this case study, uncertain parameters are assumed to deviate around design value of corresponding parameter in the engineering system and also deviations of possible values of uncertain variables are assumed to be bounded. According to this assumption, the design value of the uncertain parameter needs to be assigned to the mean value of the random set that has Gaussian distribution for probabilistic analysis and membership function distribution value of 1 for possibilistic analysis. The

assumption further necessitates both distribution functions to share the same uncertainty bounds, as shown in Figure 5.5 below. 


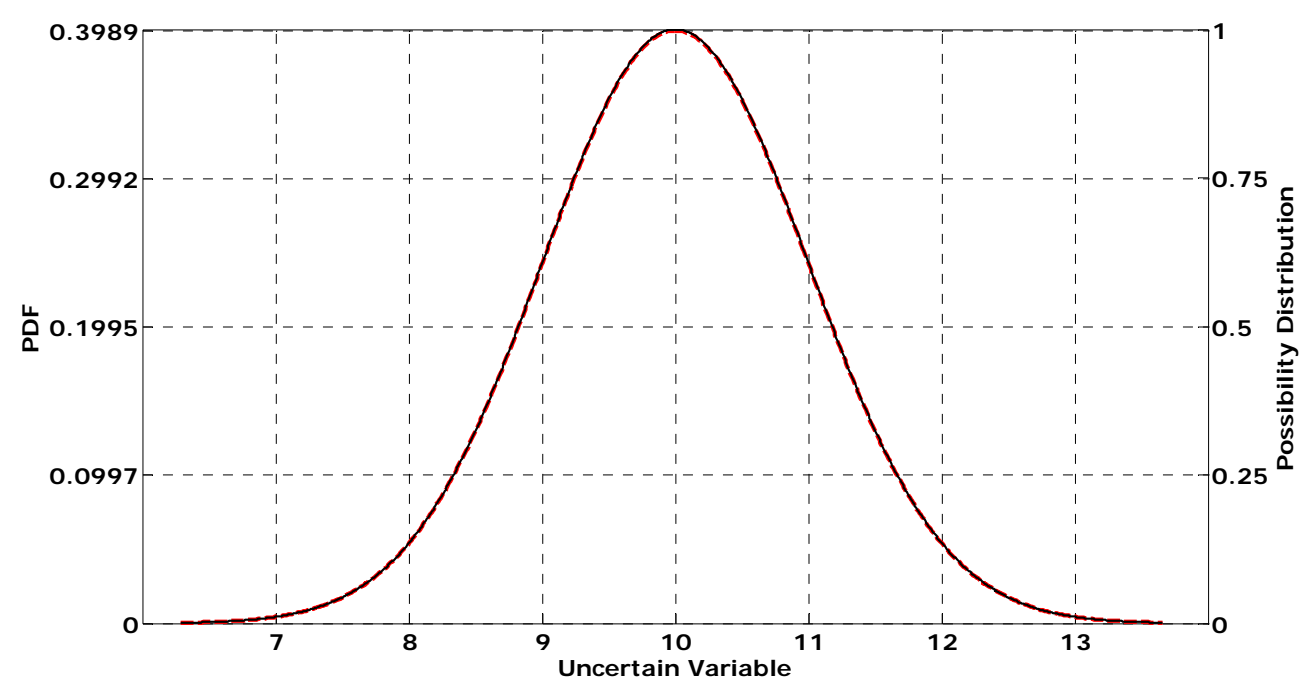

Figure 5.5: Consistency of the two uncertainty models

The assumption establishes the connection by stating the most expected values and the bounds of uncertainty ranges, only. In order to determine states in remaining levels of confidence, a distribution character of uncertainties needs to be assessed. In this case study, compatible with the mistuned bladed disk systems, stiffness parameters are assumed to have Gaussian distribution characters that are identified by standard deviations and mean values. According to the assumption, design values of stiffness parameters are assigned to the mean value of the corresponding Gaussian distributions and the extent of deviations of uncertain parameters around the design values are represented by assigned standard deviation values. Correspondingly, compatible with the interpretation of consistency principle in this study, alpha-cuts of possibility distributions of uncertain stiffness parameters are determined from the corresponding probability density functions, which is expressed mathematically as follows;

$$
\mu(x)=\frac{p(x)}{\max [p(x)]}
$$


where stands for membership function distribution of the possibility distribution and stands for the probability density function. Eq. (5.2) scales probability density functions with its maximum value resulting in a membership value of 1, where both distributions share the same uncertainty bounds. Furthermore, utilizing Eq. (5.2), alpha-cuts of possibility distributions of fuzzy stiffness variables, used in fuzzy modal analysis, are calculated, an example of which is shown in Figure 5.6. It should be noted that, possibilistic method does not require a prior knowledge of distribution of the uncertainty; however, in order to perform a valid comparison the same distribution as in the probabilistic method is used in this study.

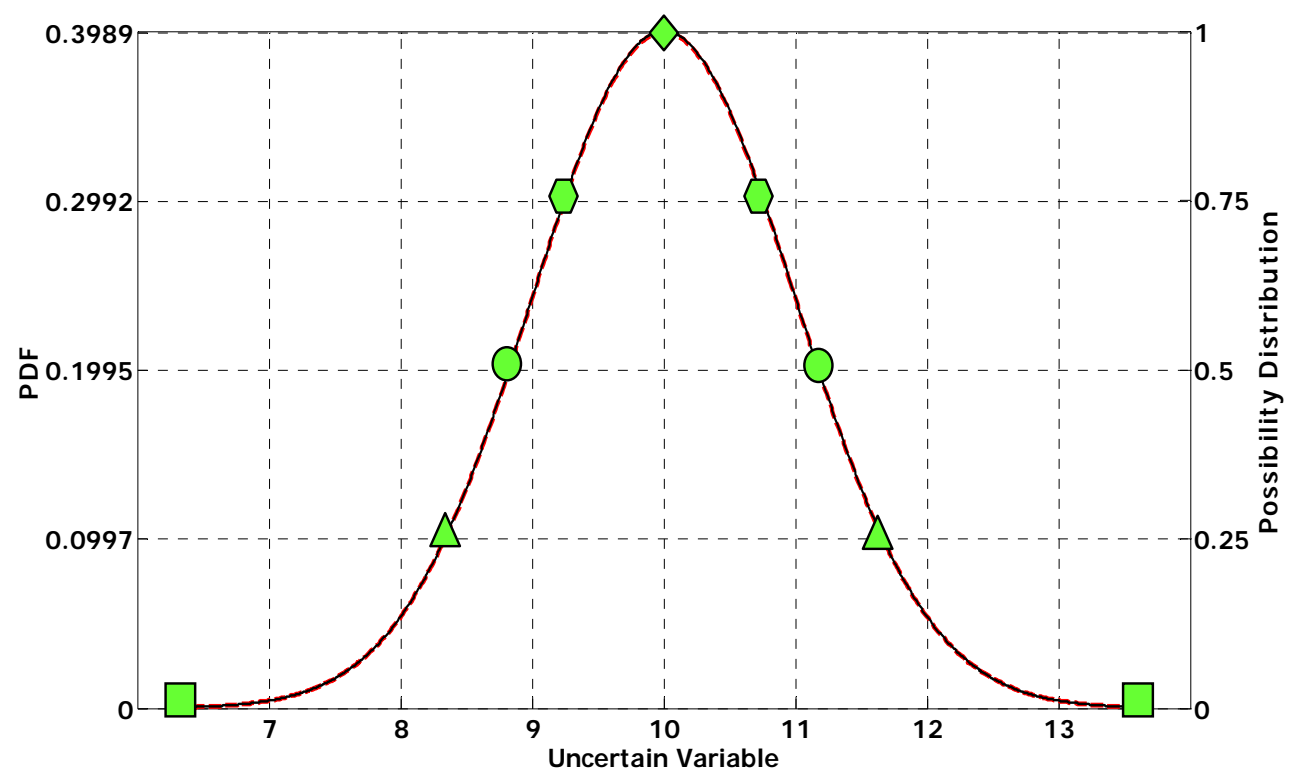

Figure 5.6: Alpha-cuts of possibility distributions 
The formulation of extension principle solution for fuzzy natural frequency and fuzzy forced vibration responses are given as follows.

$$
\begin{gathered}
\bar{\omega}[\alpha]=\left[\omega_{1}(\alpha), \omega_{2}(\alpha)\right] \\
\omega_{1}(\alpha)=\min \left\{\Psi\left(k_{11}, \ldots, k_{n n}\right) \mid k_{i j} \in k_{i j}[\alpha]\right\} \\
\omega_{2}(\alpha)=\max \left\{\Psi\left(k_{11}, \ldots, k_{n n}\right) \mid k_{i j} \in k_{i j}[\alpha]\right\} \\
\bar{q}[\alpha]=\left[q_{1}(\alpha), q_{2}(\alpha)\right] \\
q_{1}(\alpha)=\min \left\{\Phi\left(k_{11}, \ldots, k_{n n}\right) \mid k_{i j} \in k_{i j}[\alpha]\right\} \\
q_{2}(\alpha)=\max \left\{\Phi\left(k_{11}, \ldots, k_{n n}\right) \mid k_{i j} \in k_{i j}[\alpha]\right\}
\end{gathered}
$$

In the above formulation, the algorithm $\Psi$ is utilized to calculate alpha-cuts of fuzzy natural frequency parameters, $\bar{\omega}[\alpha]$, which accepts alpha-cuts of fuzzy stiffness variables $\bar{k}_{i j}[\alpha]$, and returns the upper and the lower bounds of fuzzy natural frequencies $\left[\omega_{1}(\alpha), \omega_{2}(\alpha)\right]$ at the same confidence level. Likewise, the algorithm $\Phi$ calculates alpha-cuts of fuzzy forced response parameters which accepts again alphacuts of fuzzy stiffness variables $\bar{k}_{i j}[\alpha]$ and returns the upper and the lower bounds of fuzzy forced response $\left[q_{1}(\alpha), q_{2}(\alpha)\right]$. These algorithms utilize modal analysis methods and use genetic algorithm in order to process $\min / \max$ operations in Eq. (5.3).

The ensemble of alpha-cuts of fuzzy natural frequencies constitutes the possibility distribution of the fuzzy natural frequencies and the resulting possibility distribution character is compared with character of the probability distribution obtained by probabilistic analysis for reliability considerations. Furthermore, the upper bound of the fuzzy forced response, i.e. parameter $q_{2}(\alpha)$, gives the worst-possible blade forced response in each and every confidence level, and the upper bound obtained for the least confidence level is compared with the corresponding maximum blade forced response value calculated from Monte-Carlo simulations. 


\subsubsection{Numerical Examples}

In this case study, first, a simple lumped parameter system with different number of uncertain variables is used to show the performance of the methods with increasing number of uncertain parameters. Afterwards, the performances of both uncertainty management methods are compared with each other on a lumped parameter model of a mistuned bladed disk system where all stiffness parameters as uncertain.

The first example in this case study is designed to exemplify the similarities and differences between both methods by considering different number of uncertain variables. A 10 degree-of-freedom (dof) lumped parameter model is used in this example as the vibrating system which is excited by sinusoidal excitations. The layout of the 10 dof system is shown in Figure 5.7 and the design values of mass and stiffness parameters are given in Table 5.8 and a structural damping ratio of 0.01 is used in the analysis.

The mass and stiffness values given Table 5.4 are determined by using a random normal distribution around a mean of $2 \mathrm{~kg}$ and $30000 \mathrm{~N} / \mathrm{m}$, respectively. The system is excited by two sinusoidal excitations of $100 \mathrm{~N}$ and $200 \mathrm{~N}$ amplitudes with an excitation frequency of $24.52 \mathrm{~Hz}$ (first natural frequency value) at the first and the second degrees-of-freedoms respectively. In the first part of the first example, values of the stiffness parameters $k_{1}$ and $k_{2}$ are assumed to be uncertain and have Gaussian distribution where parameters deviate around their design values with $2 \%$ standard deviation. In the second part of this example, all stiffness values are assumed to be uncertain having a normal distribution with a standard deviation of $2 \%$. 


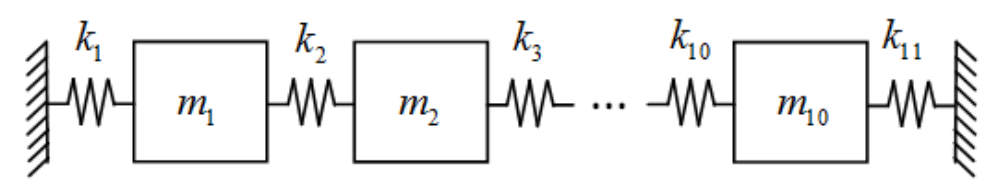

Figure 5.7: 10 dof lumped parameter model

Table 5.4 : Design values of 10 dof lumped parameter system

\begin{tabular}{|l|c|c|c|c|c|l|}
\hline Parameter & $m_{1}$ & $m_{2}$ & $m_{3}$ & $m_{4}$ & $m_{5}$ & \\
\hline Value [kg] & 2.88 & 3.45 & 3.74 & 7.21 & 3.40 & \\
\hline Parameter & $m_{6}$ & $m_{7}$ & $m_{8}$ & $m_{9}$ & $m_{10}$ & \\
\hline Value [kg] & 7.92 & 3.39 & 4.59 & 7.89 & 3.93 & \\
\hline Parameter & $k_{1}$ & $k_{2}$ & $k_{3}$ & $k_{4}$ & $k_{5}$ & \\
\hline Value [N/m] & 43687 & 32816 & 46975 & 45701 & 30577 & \\
\hline Parameter & $k_{6}$ & $k_{7}$ & $k_{8}$ & $k_{9}$ & $k_{11}$ & $k_{12}$ \\
\hline Value [N/m] & 41973 & 46461 & 43431 & 35511 & 44366 & 31767 \\
\hline
\end{tabular}

In the first example, the behaviors of the methods, as the degree of uncertainty within the system increases, are investigated. Furthermore, as a second example, for the mistuning considerations, the behavior of the methods on a cyclically symmetric lumped parameter bladed disk model is also examined. The same cyclically symmetric lumped parameter model that is used in the first case study is used and all 32 stiffness values are assumed to deviate around their design value given in Table 5.1 with a standard deviation of $0.15 \%$. In the first case study, the third engine order excitation is determined to be the worst case in the frequency range of [57-59] Hz. Correspondingly, in this example, the bladed disk is also assumed to be operating 
under $3^{\text {rd }}$ engine order excitation. The excitation frequency is $57.87 \mathrm{~Hz}$ corresponding to the ninth resonant frequency of the tuned model.

\subsubsection{Results of Comparison Study}

In this case study, two important mistuning parameters; the worst-case uncertainty range of mistuned natural frequencies and the worst-case forced response values are sought. The worst-case uncertainty ranges of mistuned natural frequencies are represented by the probability density functions (pdf) in probabilistic analysis and by the possibility distributions in possibilistic analysis. Figure 5.8 represents the performance of the methods on a simple theoretical lumped parameter model with two uncertain stiffness values. It is observed that, in this example, possibilistic and probabilistic methods converge on the same uncertainty ranges. However, this result cannot be generalized, since the performance of the methods deviates as the number of uncertain stiffness parameters increases as shown in Figure 5.9 below.

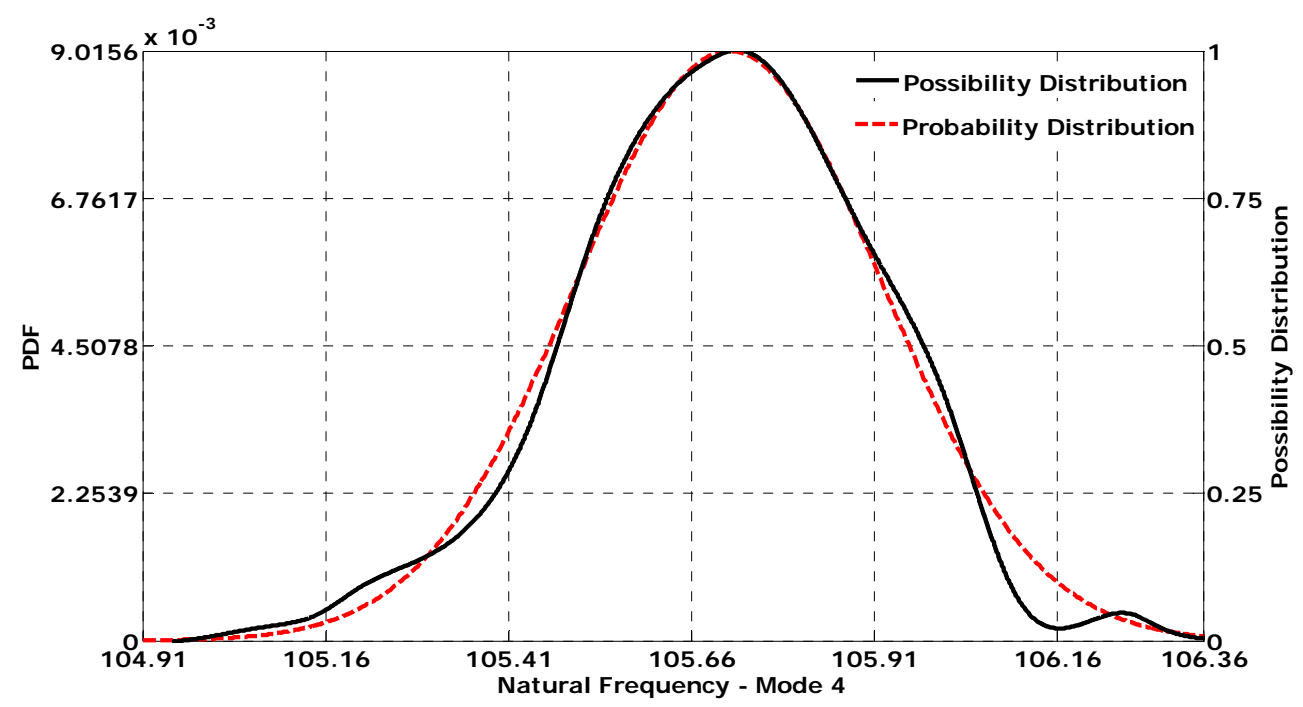


Figure 5.8 : Simple lumped parameter system $4^{\text {th }}$ natural frequency distribution- first example first part

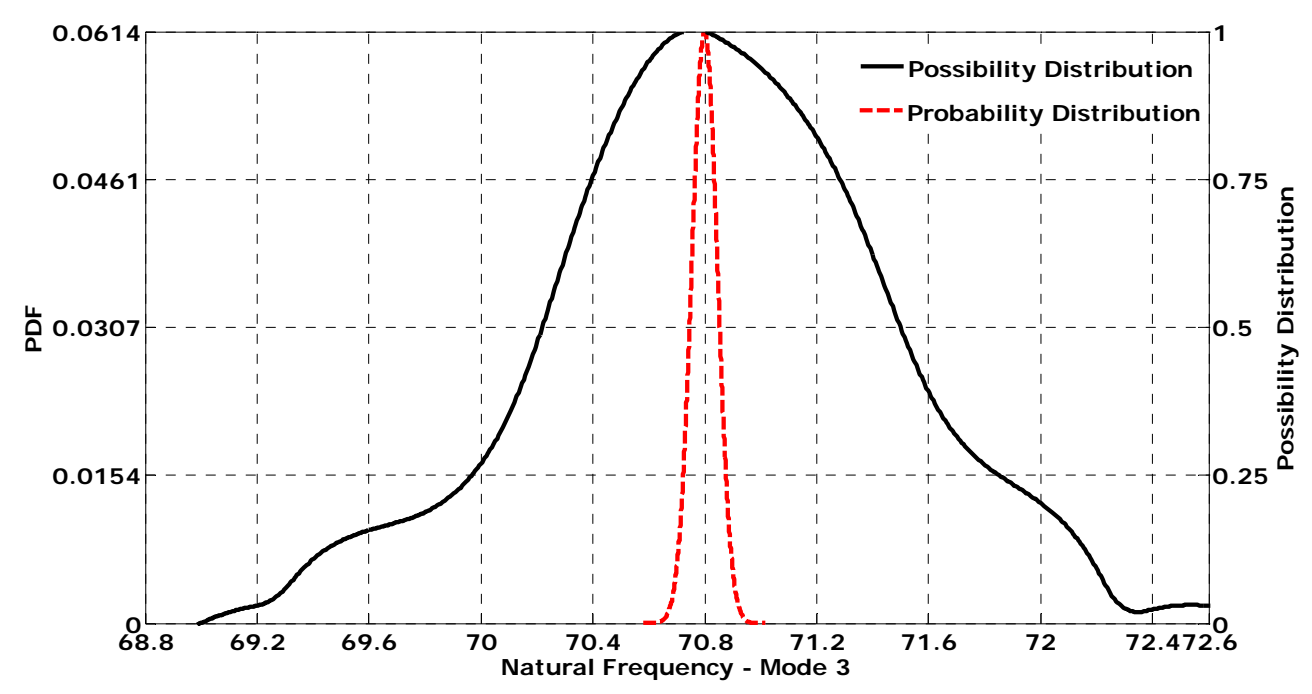

Figure 5.9 : Simple lumped parameter system $3^{\text {rd }}$ natural frequency distributions first example second part

From the results given in Figure 5.9, it is observed that the possibilistic method gives larger uncertainty ranges at each confidence level compared to the probabilistic method. Therefore, possibilistic methods are found to be more conservative and more reliable in the determination of the worst case. Correspondingly, it can be concluded that probabilistic methods are suitable for the determination of the most probable result whereas possibilistic methods should be used for the identification of the worst-possible case.

A parallel conclusion can be drawn by investigating the performances of the methods in the calculation of the worst-possible forced response values. The results for the first example obtained by fuzzy modal analysis solution and Monte-Carlo analysis with different number of iterations are compared in Figure 5.10. 


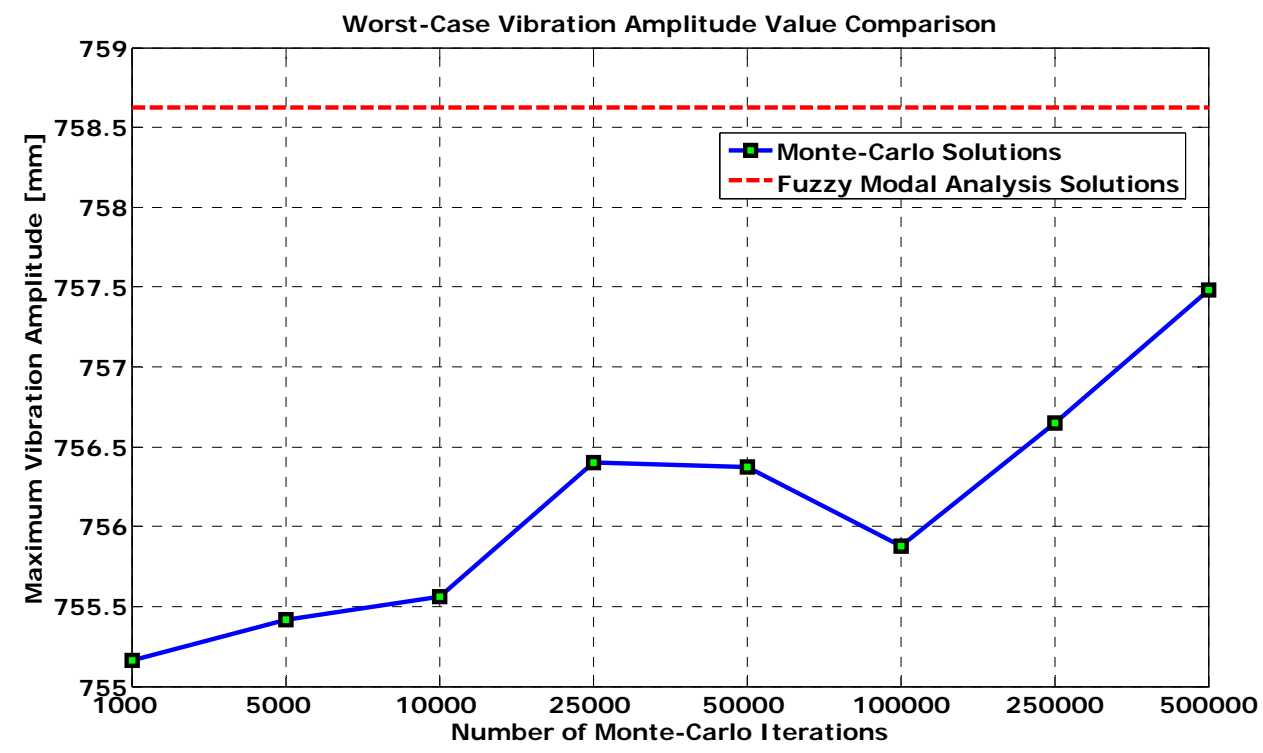

Figure 5.10 : Computational efficiency comparison - First example first part

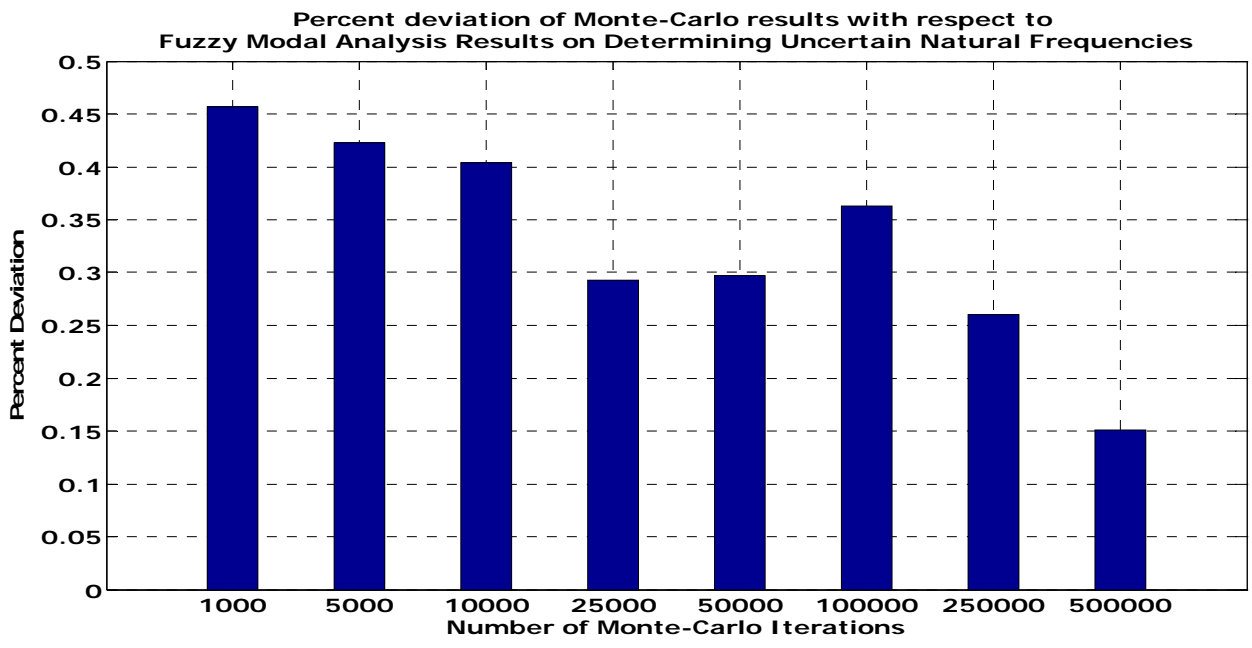

Figure 5.10 (continued) 


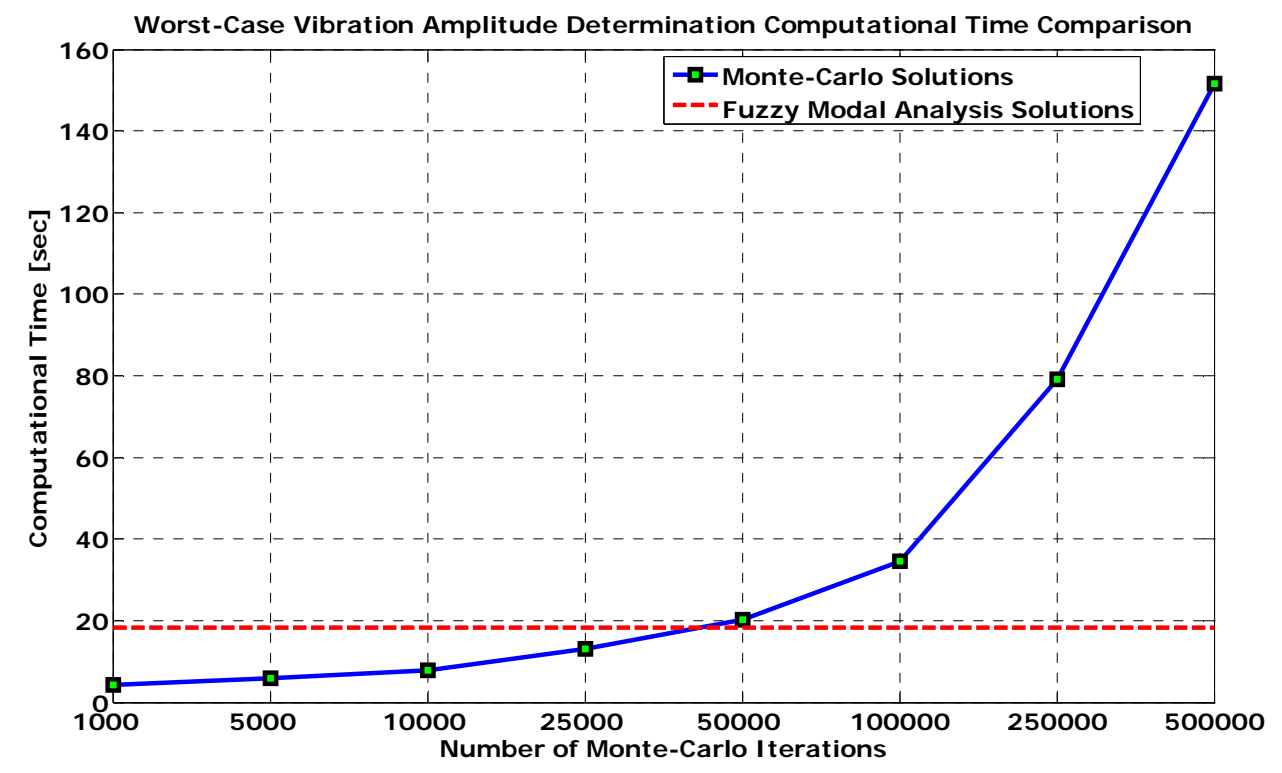

Figure 5.10 (continued)

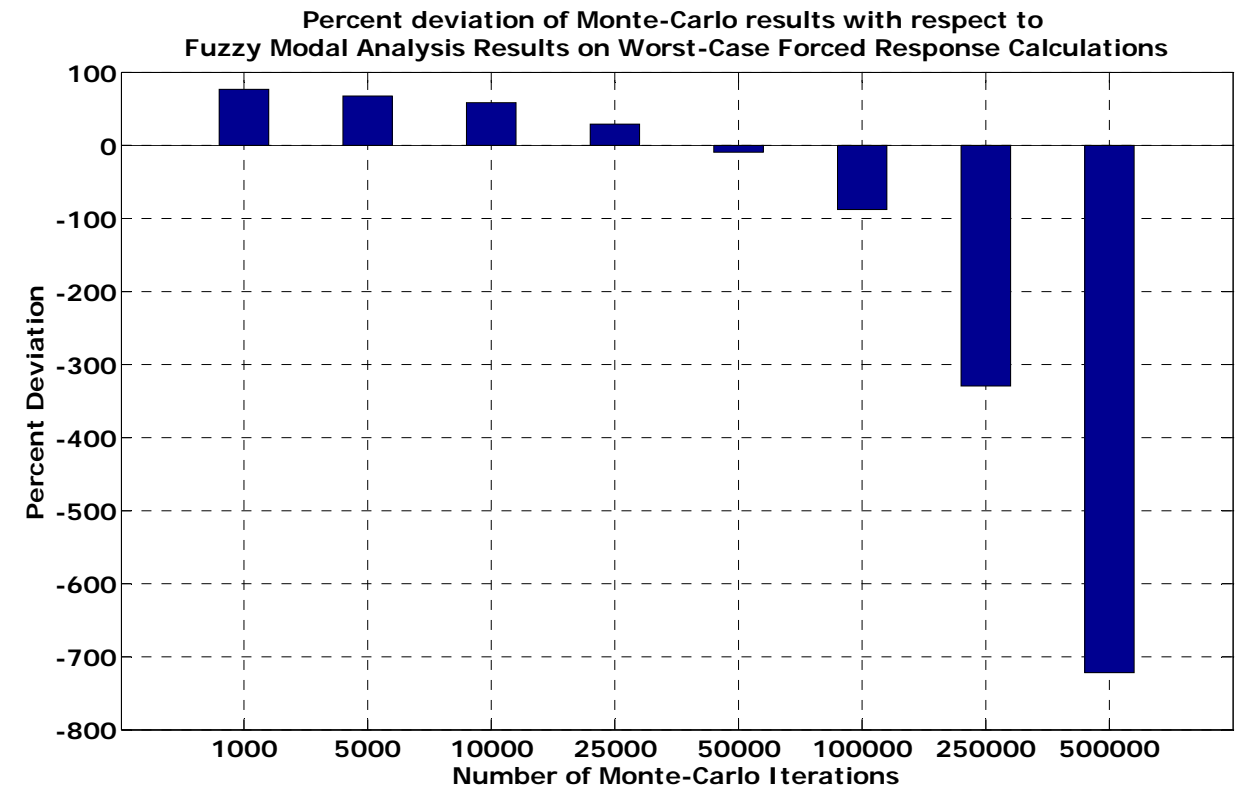

Figure 5.10 (continued) 
From Figure 5.10, it is easy to observe that for the system explained in the first example with only two uncertain parameters, probabilistic and possibilistic methods can result in the same worst-case forced response value. However, as the number of uncertain parameters increases, the possibilistic methods results in the worst-possible response requiring significantly less computational effort, as shown in Figure 5.11. More importantly, increasing the number of iterations in the Monte-Carlo analysis does not improve the results significantly. The error between 100 to 500000 iterations lies approximately at 5\%, whereas the computational time required increases more than 700 times compared to the fuzzy modal analysis.

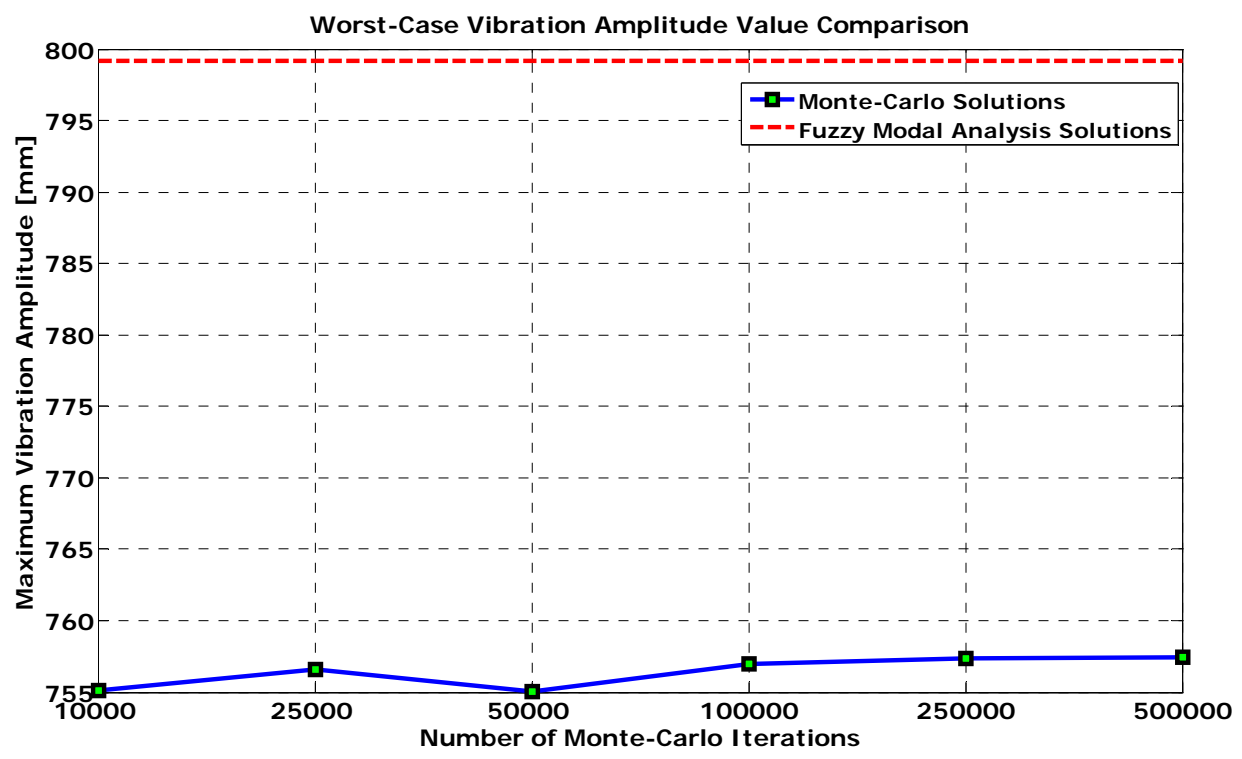

Figure 5.11 : Computational efficiency comparison - First example second part 


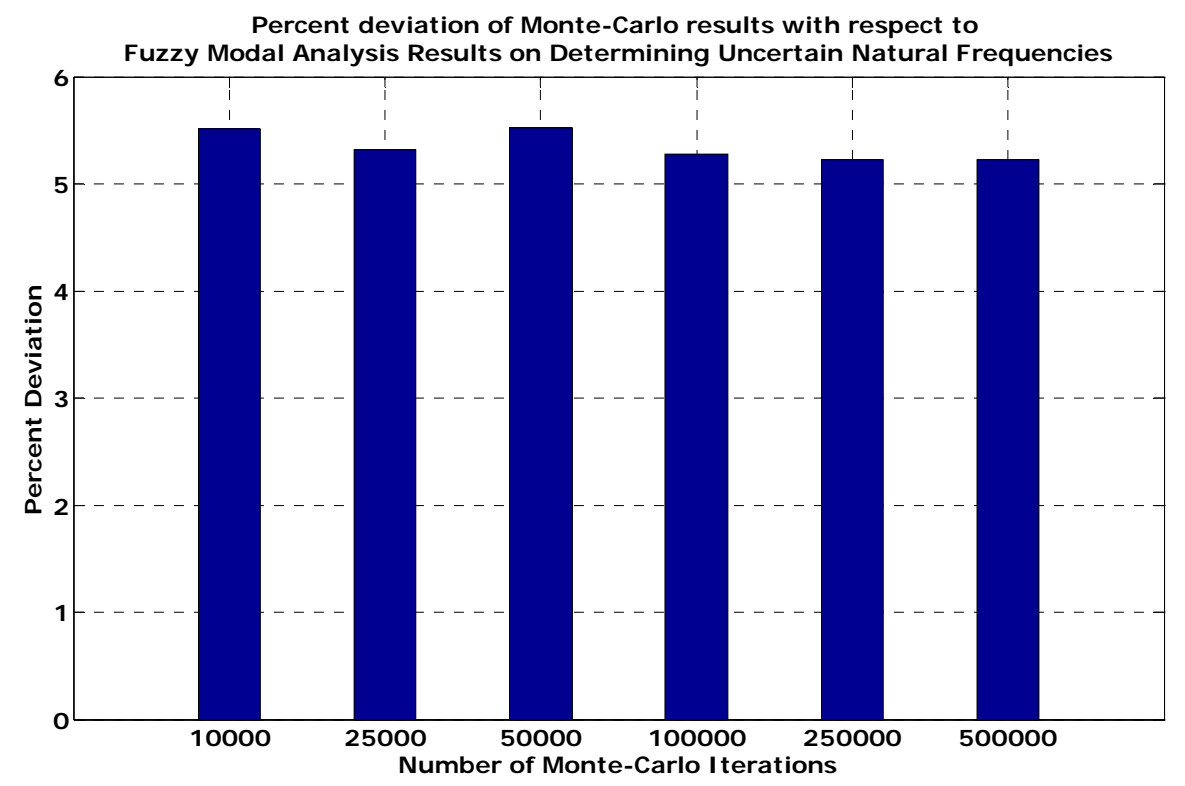

Figure 5.11 (continued)

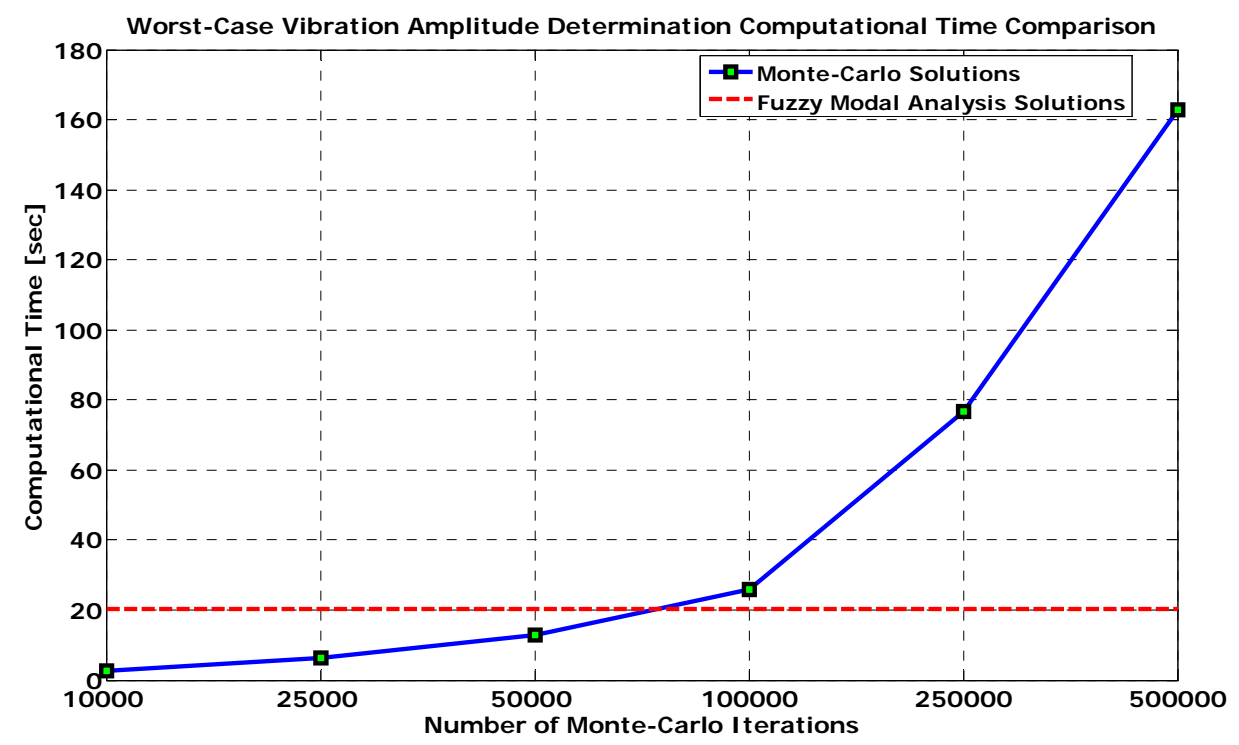

Figure 5.11 (continued) 


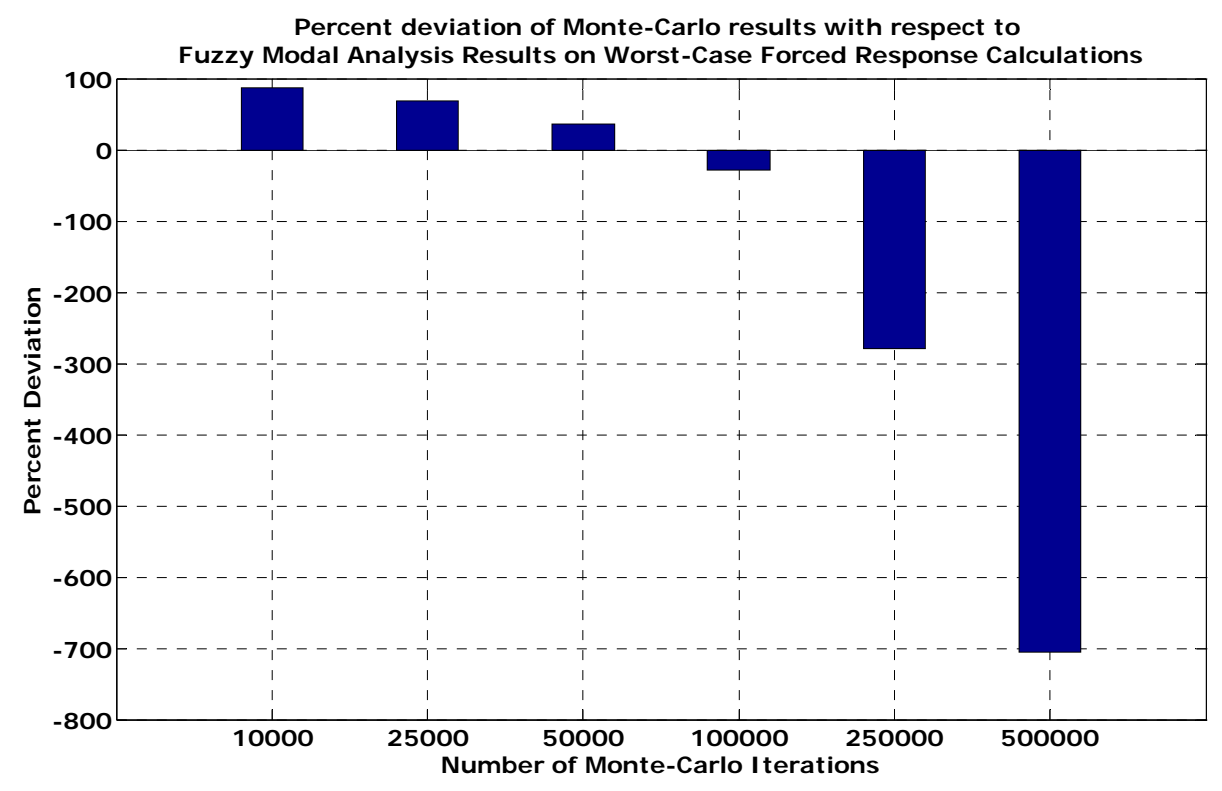

Figure 5.11 (continued)

In the second example, the same analyses are repeated on a bladed disk system with 16-blades. The reliability and computational efficiency comparison results are given in Figure 5.12 and Figure 5.13 below. It is observed that, for the bladed disk system, even though the standard deviation is much smaller than the first example, the error between the probabilistic and possibilistic methods is higher in the range of $11 \%$ to $8 \%$. Moreover, increasing the number of iterations in Monte-Carlo analysis does not result in a significant improvement in the value of the worst response, whereas the computational effort required increases more than 6000 times. This is due to the fact that the number of uncertain parameters is nearly 3 times of the first case study. 


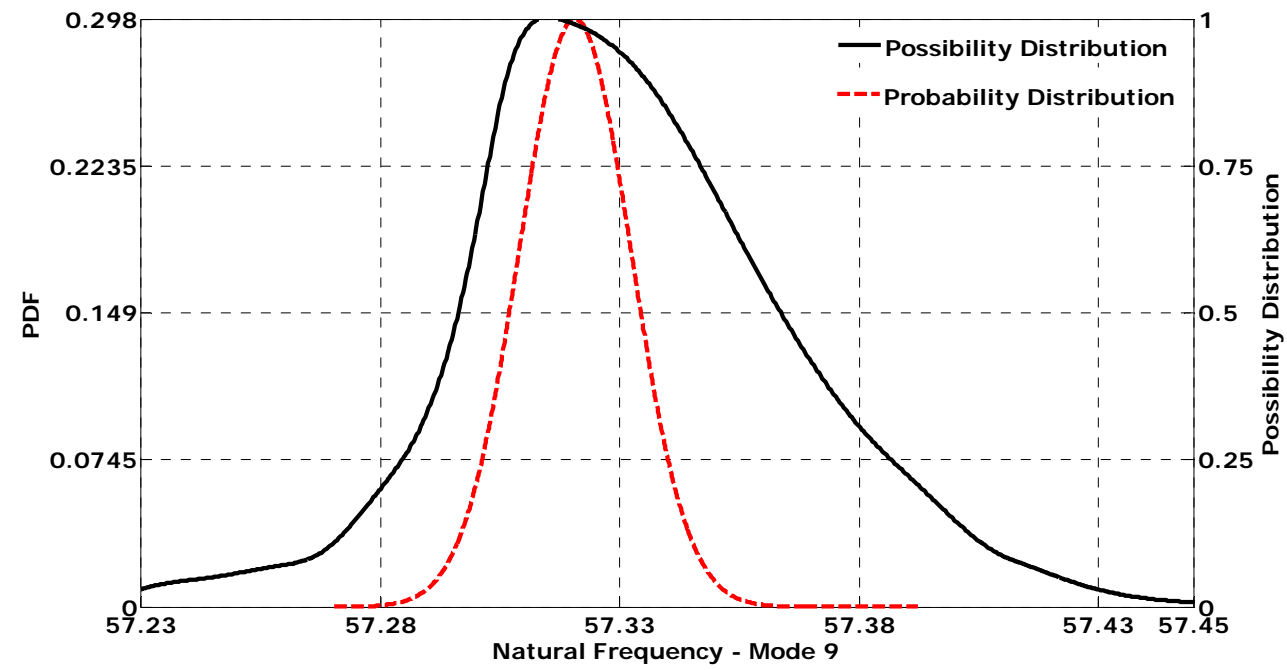

Figure 5.12 : Mistuned bladed disk model 9th natural frequency distribution

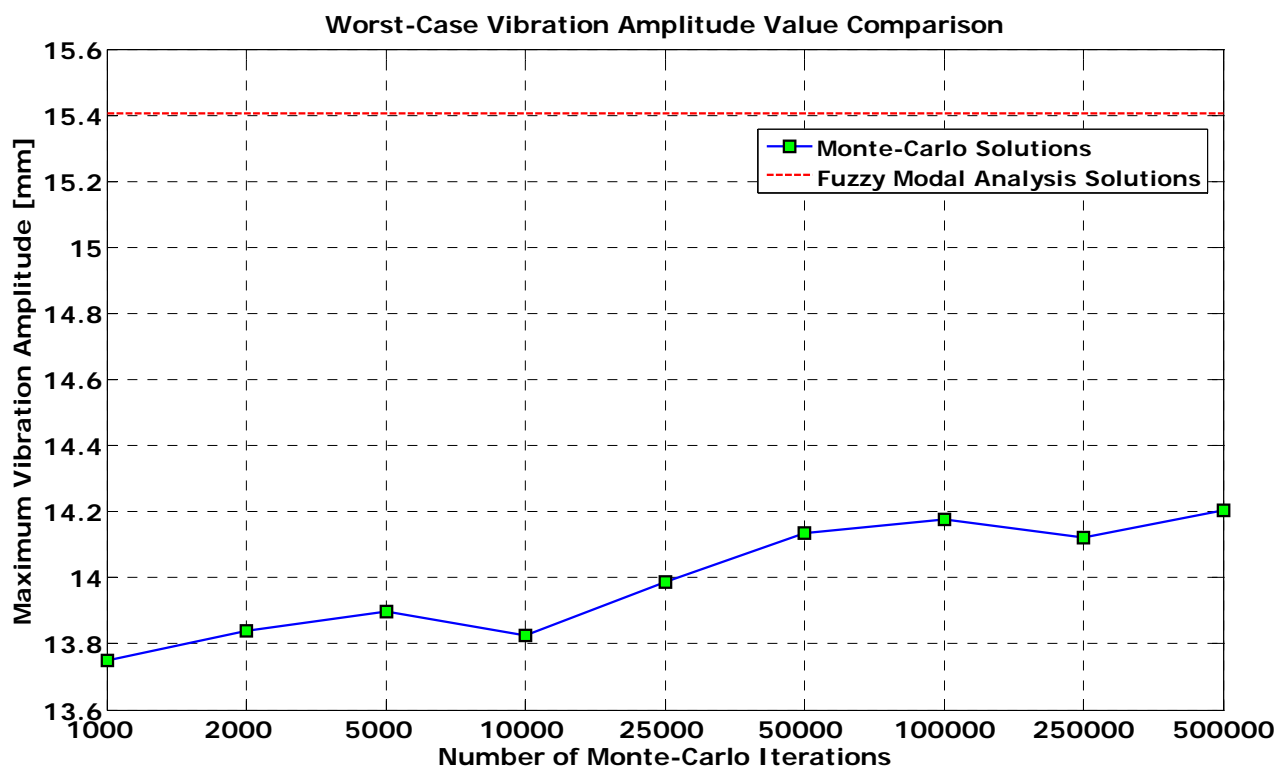

Figure 5.13 : Computational efficiency comparison - Second example 


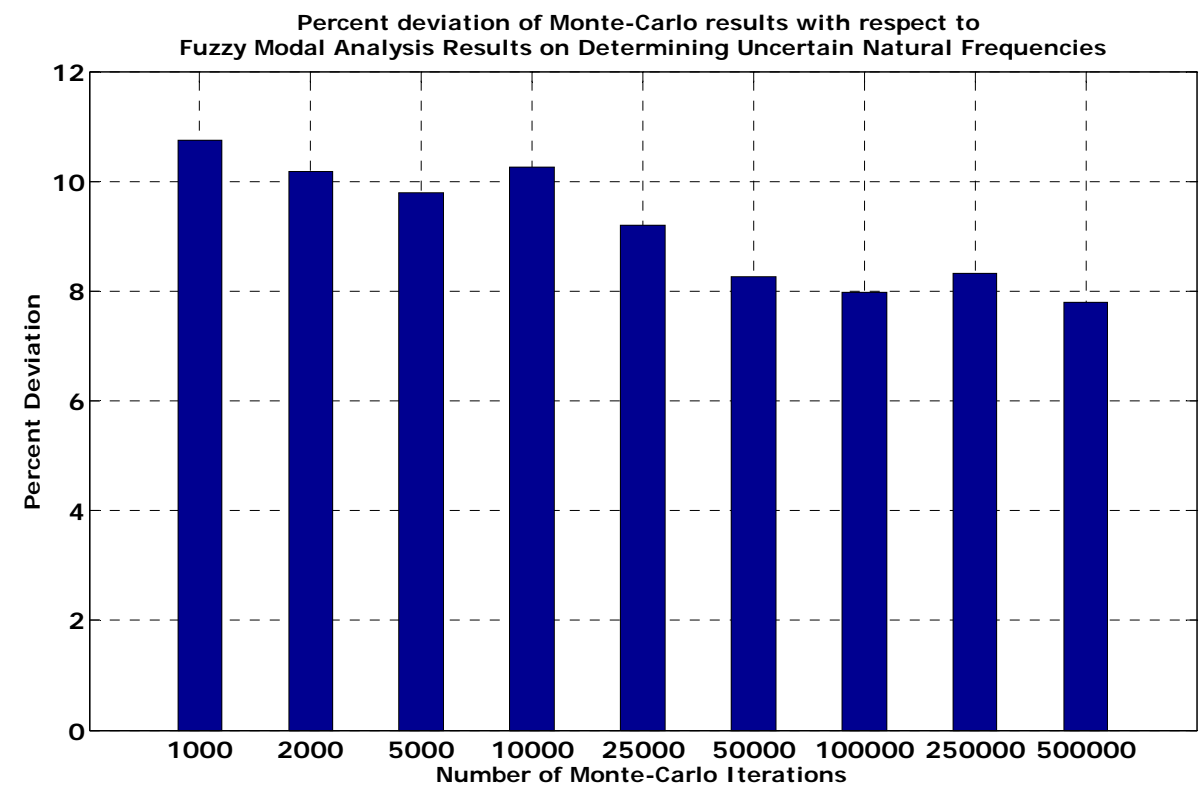

Figure 5.13 (continued)

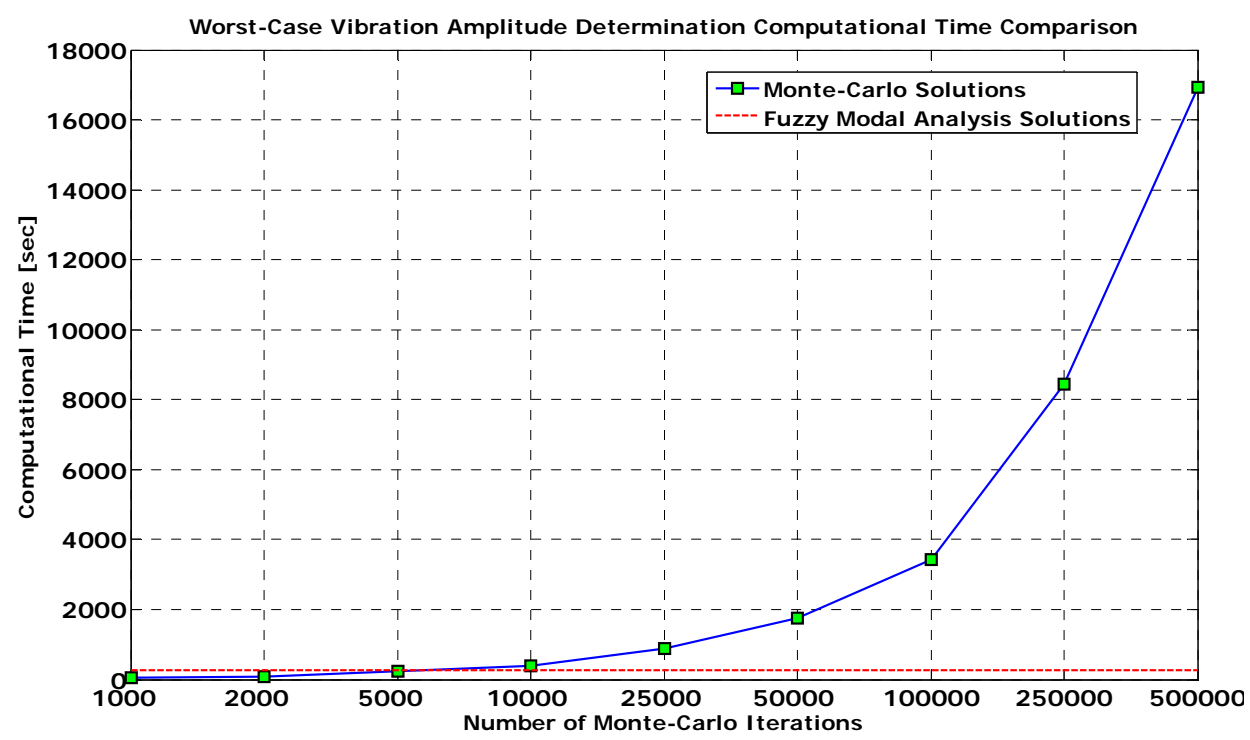

Figure 5.13 (continued) 


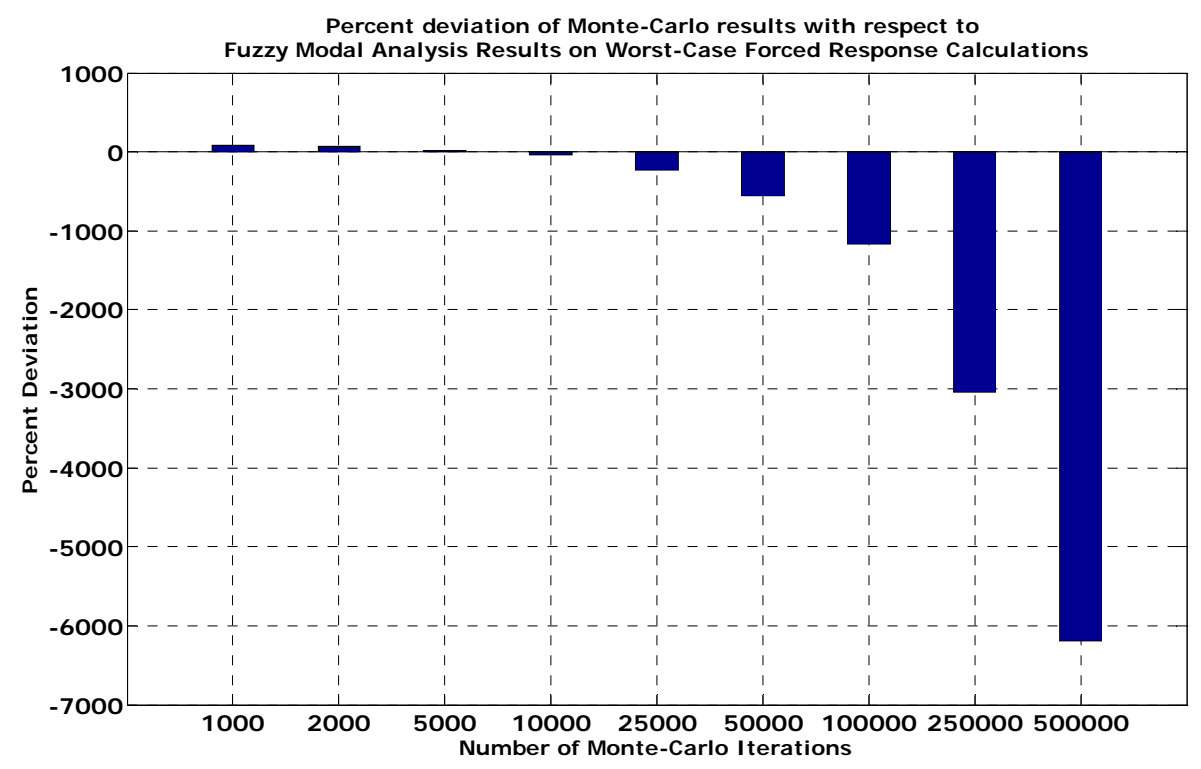

Figure 5.13 (continued)

\subsection{CASE III - Comparison between Methods in the Determination of the Worst-Case}

As stated before, in literature, first attempts on determining the worst-case mistuning effects within the uncertainty range of interest are made by probabilistic methods. However, the probabilistic methods are found to be inefficient in determining the worst-possible situation; hence, the problem is formulated as an optimization problem. Currently, in literature, two different types of optimization algorithms are utilized in order to find the global maximum forced response value within the space of possible forced response values; hybrid optimization algorithms and gradient based optimization algorithms. Hybrid algorithms are composed of a global optimization algorithm and a gradient-based algorithm. In the hybrid algorithms, first, a global optimization algorithm is utilized and consecutively a gradient based optimization algorithm which uses the results of the global optimization algorithm as 
the initial guess is utilized in order to improve the performance of the global optimization algorithm. On the contrary, gradient based optimization algorithms are faster algorithms but they require both gradient of the objective function and a 'good' initial guess in order to converge to the global extreme point. In mistuning studies, there is no known criterion for a 'good' initial guess value, therefore there is always a risk of the algorithm to converge on the local extreme points. Consequently, random combinations of mistuning patterns are utilized to find the global extreme point. In this thesis study, apart from the mentioned methods, a new method is proposed for determining the worst-case response that is possible to occur in a mistuned bladed disk system, and the results obtained with this method are compared to hybrid optimization algorithm solutions.

Hybrid algorithms require upper and lower bounds of values of uncertain parameters to constitute following optimization target:

$$
\max \left\{\varphi\left(t_{1}, \ldots, t_{n}\right) \mid a_{i}<t_{i}<b_{i}\right\}, i=1 \ldots n,
$$

where $i$ is the mistuning parameter index, $t_{i}$ is the $i^{\text {th }}$ mistuning parameter, $a_{i}$ and $b_{i}$ are the lower and upper bounds of the $i^{\text {th }}$ mistuning parameter, $\varphi$ is the algorithm that computes forced response values for a given set of mistuning parameters and $n$ is the number of mistuning parameters. The interval for each mistuning parameter used in Eq. (5.4) is equivalent to the interval of values of mistuning parameters at $0^{\text {th }}$ alpha cut of corresponding membership function distribution in possibilistic analysis. Hence, for worst-case determination purposes the first step of the hybrid algorithm, i.e. global optimization algorithm solution and the method of possibilistic analysis that utilizes genetic algorithm which is used in the first and second case studies of this thesis are equivalent. In this case study, another way to utilize possibilistic methods in determining the worst-case forced response is proposed which is more accurate and computationally more efficient. Hence, from now on, in this case study the term possibilistic analysis is referred to possibilistic analysis method utilizing gradient-based algorithm, only. 
In the proposed method, only a nonlinear unconstrained optimization algorithm which is a gradient-based optimization algorithm is utilized. The proposed method achieves to determine 'good' initial guess values for each and every confidence level utilizing the method of homotophy continuation. It uses the results of the previous level of confidences in order to come up with a logical initial guess value for the current level of confidence iteration. This philosophy relies on the fact that the values that an uncertain variable can take at a level of confidence contain the values that the same uncertain variable can take at previous confidence levels. Hence, basically, the worst-case solution of a level of confidence can be used as a good initial guess for the next level of confidence. Furthermore, the results of previous level of confidences can be combined and processed in order to determine better initial guess values for the next level of confidence. In this case study, performances of the possibilistic analysis method utilizing two different algorithms for determining the initial guess values and the next level of confidence are investigated.

In this case study, the performances of the hybrid optimization algorithm and the possibilistic analysis method are compared on a more realistic case constructed by a reduced order finite element model of a compressor bladed disk. The finite element model that has been used by Orbay [24] is utilized in this case study. The rotor has 36 blades and a nominal drum diameter of $310 \mathrm{~mm}$. The blades have shrouds at the middle and they are modeled with a twist angle to demonstrate a realistic compressor rotor. The material of the bladed disk is chosen to be titanium-nickel alloy with the properties given in Table 5.5 and the geometry of the bladed disk is shown in Figure 5.14 and Figure 5.15 .

Table 5.5: Physical properties of reduced order finite element model used

\begin{tabular}{|c|c|}
\hline Property & Value \\
\hline Density & $8400 \mathrm{~kg} / \mathrm{m}^{3}$ \\
\hline Modulus of Elasticity & $270 \mathrm{GPa}$ \\
\hline Poisson's Ratio & 0.3 \\
\hline
\end{tabular}




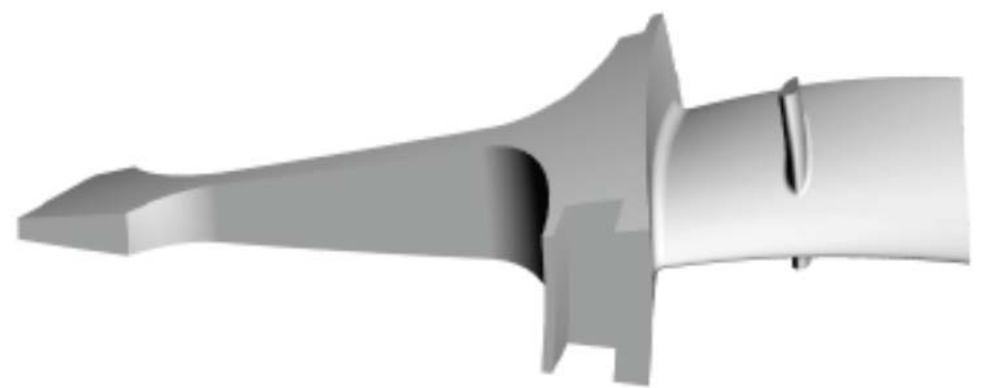

Figure 5.14: Fundamental sector geometry [24]

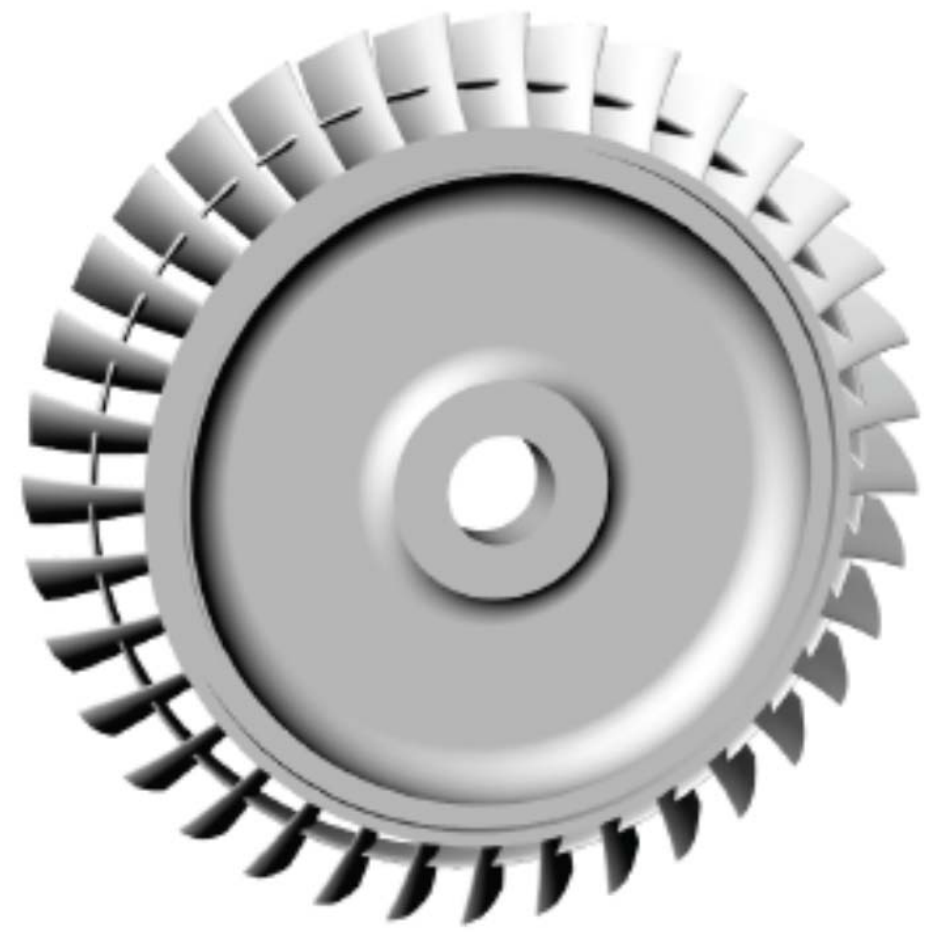

Figure 5.15: The bladed-disk geometry [24] 
The finite element model of the fundamental sector has 1190 number of 4-noded tetrahedral elements with 415 nodes. The shrouds between blades are assumed to be free and the boundary conditions are modeled accordingly. The bladed disk is clamped only on inner radius nodes. In this study the compressor rotor is assumed to be excited by engine order of order 3 at an excitation frequency of $213 \mathrm{rad} / \mathrm{s}$ [24]. The engine order forcing, which is applied in axial direction at the tip nodes of the blade, has a total magnitude of $12 \mathrm{~N}$. Hence, for this case study, the tuned blade vibration amplitude is calculated as $23.55 \mathrm{~mm}$.

The performances of methods in worst-case determination are investigated using two examples. In the first example, the first and the nineteenth blades are assumed to be affected by mistuning and the modal blade stiffness values (square of the cantilevered blade natural frequencies) are assumed to be deviating at most $\pm 5 \%$. The case is solved in three different ways: possibilistic analysis utilizing gradient-based optimization method, directly by genetic optimization method (which is the same as possibilistic analysis utilizing genetic optimization algorithm) and brutal force. Possibilistic and direct optimization methods are compared with each other in terms of computational efficiency and accuracy of the results obtained using the global maximum value that is determined from results of brutal force solution.

In the second example, the performance of the possibilistic method is tested on a realistic case study in which all blades are assumed to be mistuned. In the mistuned structure, blade modal stiffness values (square of the cantilevered blade natural frequency values) are assumed to be deviating at most $\pm 5 \%$ from the tuned value. In order to determine the worst blade vibration amplitude that is possible to occur within the given uncertainty interval, different options of the gradient-based algorithm and hybrid optimization algorithms are utilized. 


\subsubsection{Numerical Results}

For the first example where the cantilevered blade natural frequencies of only two of the blades; i.e. blade \#1 and blade \#19, are assumed to be imprecise, it is possible to determine the maximum possible blade forced response value for all possible mistuning pattern configurations, as shown in Figure 5.16.

From Figure 5.16, it can be concluded that for the given range of mistuning the maximum possible blade vibration amplitude is $27.22 \mathrm{~mm}$, which occurs when mistuning coefficients of blade \#1 and blade \#19 are both 1.05 as shown by red star in Figure 5.16. In mathematical terms, $27.22 \mathrm{~mm}$ blade vibration amplitude is the global maximum value in the range of $\pm 5 \%$ mistuning considered; hence, it is the target value for the methods used for the determination of the worst-case. The performances of the algorithms used for the determination of the worst-case are compared in Table 5.6, where the options given in Table 5.7 are used for the genetic algorithm and the gradient based optimization algorithm.

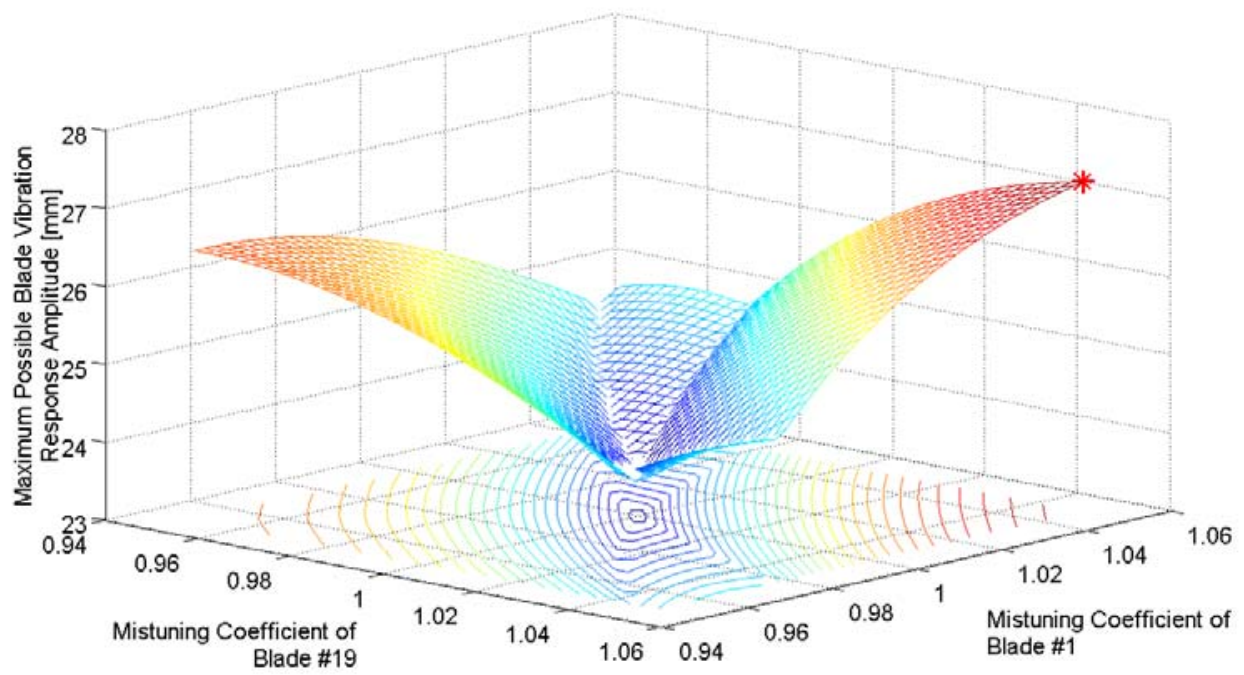

Figure 5.16: Maximum blade vibration values for all possible mistuning pattern configurations of blade \#1 and blade \#19 
Table 5.6: Performances of the algorithms used for the determination of the worstcase

\begin{tabular}{|c|c|c|}
\hline & $\begin{array}{c}\text { Possibilistic } \\
\text { Method Solution }\end{array}$ & $\begin{array}{c}\text { Global Optimization } \\
\text { Solution }\end{array}$ \\
\hline $\begin{array}{c}\text { Maximum Blade Displacement } \\
\text { Values [mm] }\end{array}$ & 27.22 & 26.44 \\
\hline Amplification Factor & 1.16 & 1.12 \\
\hline $\begin{array}{c}\text { Total Number of Required } \\
\text { Function Evaluations }\end{array}$ & 39 & 3900 \\
\hline
\end{tabular}

Table 5.7: Options of optimization algorithms used

\begin{tabular}{|c|c|c|c|}
\hline \multicolumn{2}{|c|}{$\begin{array}{c}\text { Gradient Based Optimization } \\
\text { Algorithm Options }\end{array}$} & \multicolumn{2}{c|}{ Algorithm Options } \\
\hline Option Name & Value & Option Name & Value \\
\hline Maximum Iterations & 400 & Generations & 5000 \\
\hline $\begin{array}{c}\text { Maximum Function } \\
\text { Evaluations }\end{array}$ & 3600 & Population Size & 75 \\
\hline Input Tolerance & $1 \mathrm{e}-06$ & Time Limit & Infinite \\
\hline Output Tolerance & $1 \mathrm{e}-06$ & Fitness Limit & - Infinite \\
\hline & & Stall Generations & 50 \\
\hline & & Stall Time Limit & 20 \\
\hline & & Function Tolerance & $1 \mathrm{e}-06$ \\
\hline
\end{tabular}

Genetic optimization is run several times and the one that results in the maximum blade vibration amplitude is given in Table 5.6. From these results, it is evident that the possibilistic solution approach (utilizing gradient based optimization) is capable of determining the global maximum result but global optimization algorithm (i.e. genetic optimization) fails to converge on the maximum blade vibration amplitude even if it requires 100 times more computational time compared to the possibilistic analysis method. The computational time required is determined by the number of function evaluations, since it is the parameter that effects the computational time the most. 
In the second example, the performances of hybrid optimization algorithm and gradient-based possibilistic approach are tested on a more realistic case where all of the blades are assumed to be mistuned. In this example, the options of genetic and gradient-based optimization algorithms are kept the same as the options in the first example. The results obtained by both methods are presented in Table 5.8, Table 5.9, Table 5.10 in detail. The details of both the genetic algorithm and the consecutive gradient based algorithm are given in Table 5.8 and Table 5.9, respectively. Then the results of possibilistic approach utilizing two different initial guess determination methods are detailed in Table 5.10.

Table 5.8: Performances of worst-case determination methods for the second example - First step of the hybrid algorithm

\begin{tabular}{|c|c|c|c|}
\hline \multicolumn{4}{|c|}{ Global Optimization Algorithm Solution } \\
\hline Trial & $\begin{array}{c}\text { Maximum Possible } \\
\text { Displacement [mm] }\end{array}$ & $\begin{array}{c}\text { Number of } \\
\text { Function } \\
\text { Evaluations }\end{array}$ & $\begin{array}{c}\text { Amplification } \\
\text { Factor }\end{array}$ \\
\hline 1 & 30.57 & 3975 & 1.298 \\
\hline 2 & 30.20 & 4575 & 1.282 \\
\hline 3 & 30.63 & 4125 & 1.301 \\
\hline 4 & 30.30 & 5925 & 1.287 \\
\hline 5 & 30.02 & 3900 & 1.275 \\
\hline
\end{tabular}

Table 5.9: Performances of worst-case determination methods for the second example - Last step of the hybrid algorithm

\begin{tabular}{|c|c|c|c|c|c|}
\hline \multicolumn{2}{|c|}{ Trial Obal Optimization Algorithm } & \multicolumn{3}{|c|}{ Hybrid Algorithm } \\
\hline $\begin{array}{c}\text { Maximum } \\
\text { Displacement } \\
{[\mathrm{mm}]}\end{array}$ & $\begin{array}{c}\text { Amplification } \\
\text { Factor }\end{array}$ & $\begin{array}{c}\text { Maximum } \\
\text { Displacement } \\
{[\mathrm{mm}]}\end{array}$ & $\begin{array}{c}\text { Total } \\
\text { Number of } \\
\text { Function } \\
\text { Evaluations }\end{array}$ & $\begin{array}{c}\text { Amplification } \\
\text { Factor }\end{array}$ \\
\hline 1 & 30.57 & 1.298 & 31.27 & 5316 & 1.328 \\
\hline 2 & 30.20 & 1.282 & 31.27 & 5916 & 1.328 \\
\hline 3 & 30.63 & 1.301 & 31.27 & 5271 & 1.328 \\
\hline 4 & 30.30 & 1.287 & 31.27 & 5401 & 1.328 \\
\hline 5 & 30.02 & 1.275 & 31.27 & 5572 & 1.328 \\
\hline
\end{tabular}


Table 5.10: Performances of worst-case determination methods for the second example - Possibilistic Approach

\begin{tabular}{|c|c|c|c|c|}
\hline \multicolumn{5}{|c|}{ Possibilistic Method Solution } \\
\hline $\begin{array}{c}\text { Initial Guess } \\
\text { Metermination }\end{array}$ & $\begin{array}{c}\text { Maximum } \\
\text { Possible } \\
\text { Displacement } \\
{[\mathrm{mm}]}\end{array}$ & $\begin{array}{c}\text { Total Number of } \\
\text { Function } \\
\text { Evaluations }\end{array}$ & $\begin{array}{c}\text { Total Number } \\
\text { of Iterations }\end{array}$ & $\begin{array}{c}\text { Amplification } \\
\text { Factor }\end{array}$ \\
\hline 1 & 31.27 & 2934 & 71 & 1.328 \\
\hline 2 & 31.27 & 5731 & 132 & 1.328 \\
\hline
\end{tabular}

From Table 5.8, it is observed that due to the random nature of the genetic algorithm the results vary at each iteration and there is no correlation between the accuracy of the result and the computation time, i.e. number of function evaluations. Consequently, for the second step of the hybrid algorithm, mistuning patterns resulting blade displacement amplitudes given in Table 5.8 are used as the initial guess of the unconstrained nonlinear optimization algorithm, and the results obtained are presented in Table 5.9. From Table 5.9, it is observed that for the mistuning case considered, all initial guess values converged on the same mistuned blade forced response value, which is $31.27 \mathrm{~mm}$. The results obtained by possibilistic analysis method are given in Table 5.10. Both of the ways to determine the next level of confidence explained in Section 4.3.3 are utilized. In this case study, in order to establish a criterion for determining the next level of confidence, for both of the methods, initially, calculations for confidence levels of 0.99 and 0.95 are made. This convention makes the first alpha-cut step, which is a parameter used in algorithms to determine the next level of confidence, to be $0.99-0.95=0.04$. Compatible with the convention of membership function distributions, the mistuning coefficient initial guess value for the iteration at alpha-cut 0.99 needs to be 1 , i.e. the tuned case, for all blades. Initially, the mistuning pattern that results in the worst-case situation at 0.99 level of confidence is calculated and recorded together with the number of iterations made in order to converge to the worst-case forced response value. For the next level of confidence, i.e. 0.95, the initial guess values for each blade are determined by linear extrapolation using the last two solutions; for instance, for 0.95 they are 0.99 
and 1. Finally, the mistuning pattern that results in the worst-case for the level of confidence 0.95 is determined and recorded together with the number of iterations made in order to converge to the worst-case forced response value. The collected information from calculations of level of confidence 0.99 and 0.95 is processed to determine the next level of confidence by two different algorithms as stated in Section 4.3.3.

In the first row of the results given in Table 5.10, the next level of confidence is determined by how close the initial guess values of the previous level of confidence are to the values of the mistuning coefficients that result in the worst-case situation in the previous level of confidence. Namely, let us suppose that the vector of initial guess values for the level of confidence 0.99 to be $\left\{I G_{1}\right\}$ and the corresponding mistuning pattern that results in the worst-case situation at level of confidence 0.99 to be $\left\{\right.$ MistPattern $\left._{1}\right\}$. Similarly, for the next level confidence the parameters are called to be $\left\{I G_{2}\right\}$ and $\left\{\right.$ MistPattern $\left.n_{2}\right\}$. Then, the next level of confidence after the level of confidence 0.95 is determined by either increasing the alpha-cut step by two times the initial alpha-cut step, i.e. 0.08 , or decreasing it to half, i.e. 0.02 . If the maximum difference between the initial guess and the concluding mistuning pattern at level of confidence 0.95 is equal to or smaller than the one calculated for the level of confidence 0.99 ; i.e., if the following condition is satisfied,

$$
\begin{aligned}
& \max \left(\text { abs }\left(\left\{\text { MistPattern }_{2}\right\}-\left\{I G_{2}\right\}\right)\right) \\
& \leq \max \left(\operatorname{abs}_{\left.\left(\left\{\text {MistPattern }_{1}\right\}-\left\{I G_{1}\right\}\right)\right)},\right.
\end{aligned}
$$

the alpha-cut step is increased to twice of the previous alpha-cut step or it is decreased to the half of the previous alpha-cut step otherwise. Hence, if the first condition is satisfied, the next level of confidence is determined to be $0.87=0.95-$ $0.04 * 2$, otherwise it is determined to be $0.93=0.95-0.04 / 2$. Consequently, the 
oncoming level of confidence values are determined by the same algorithm for the previous two levels of confidence.

A similar algorithm is utilized for the last row of results given in Table 5.10. The next level of confidence is determined by checking the number of iterations done at the previous levels of confidence. Let us suppose that the parameters Iterations 1 and Iterations ${ }_{2}$ represent the number of iterations made for the calculation of the worst possible blade forced response values at levels of confidence 0.99 and 0.95 respectively. Then the next level of confidence after level of confidence 0.95 is determined by checking whether Iterations ${ }_{2}$ is bigger or smaller than Iterations . $_{\text {. }}$ If the number of iterations are increased between the first and the second level of confidence calculations, the next level of confidence is determined by decreasing the alpha-cut step as half of the initial alpha-cut step or if the number of iterations required is decreased than the next level of confidence is determined by decreasing the alpha-cut step by twice of the initial alpha-cut step. For both of the algorithms, after the determination of the next alpha-cut, the corresponding initial guess value is determined by linear extrapolation as stated before.

According to the results given in Table 5.10, the second initial guess determination strategy resulted in the same forced response value as the hybrid algorithm does, requiring a similar number of function evaluations; however, using the first initial guess determination strategy the possibilistic approach resulted in the same mistuned blade forced response value requiring at least 1.8 times less computational time. The performances of the two strategies mentioned at each and every confidence level are visualized in Figure 5.17 and Figure 5.18. From these figures it is obvious that possibilistic method requires 6 alpha-cuts for the first strategy and 15 alpha-cuts for the second strategy and this is the reason for the second strategy to be computationally more expensive. 


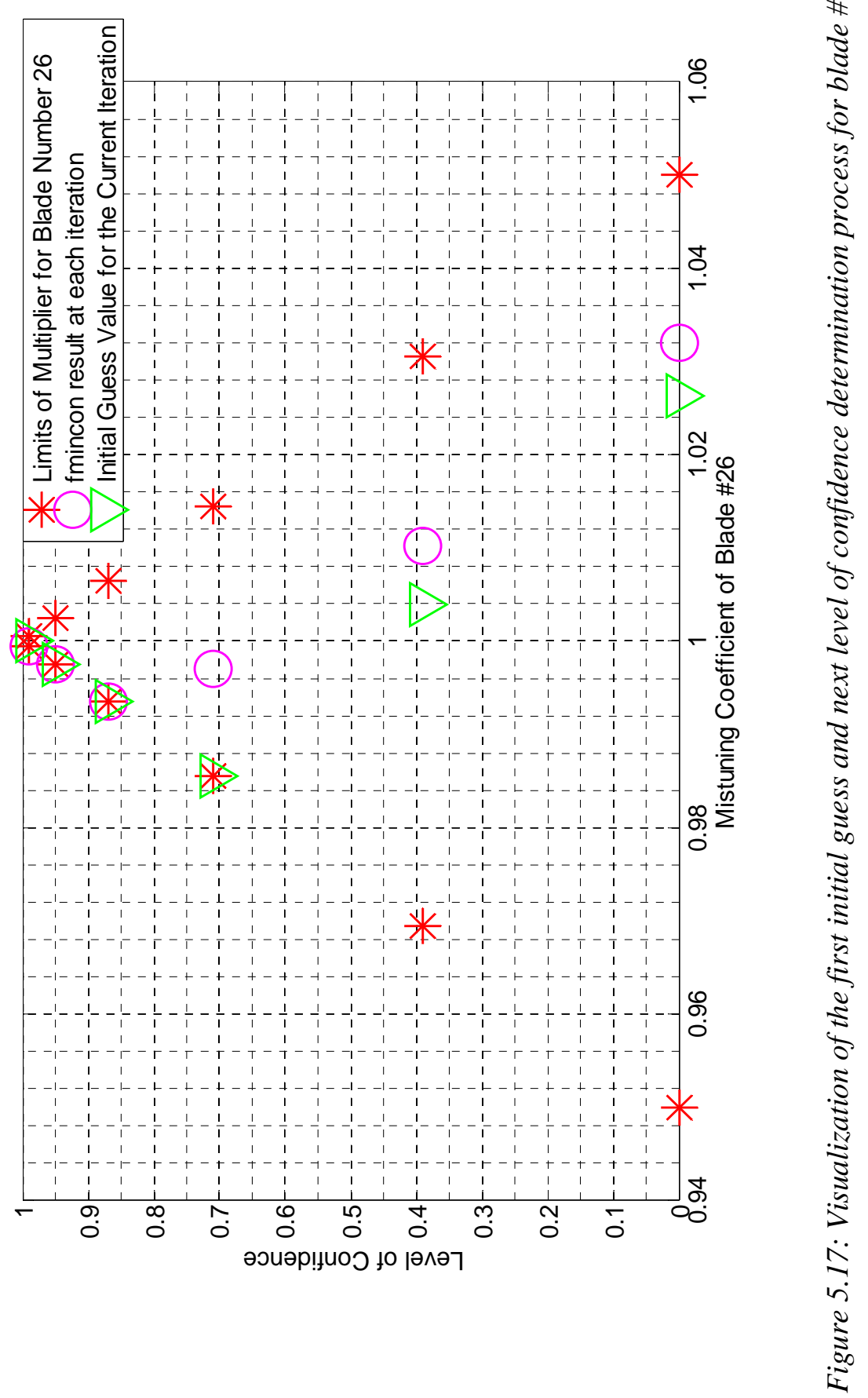




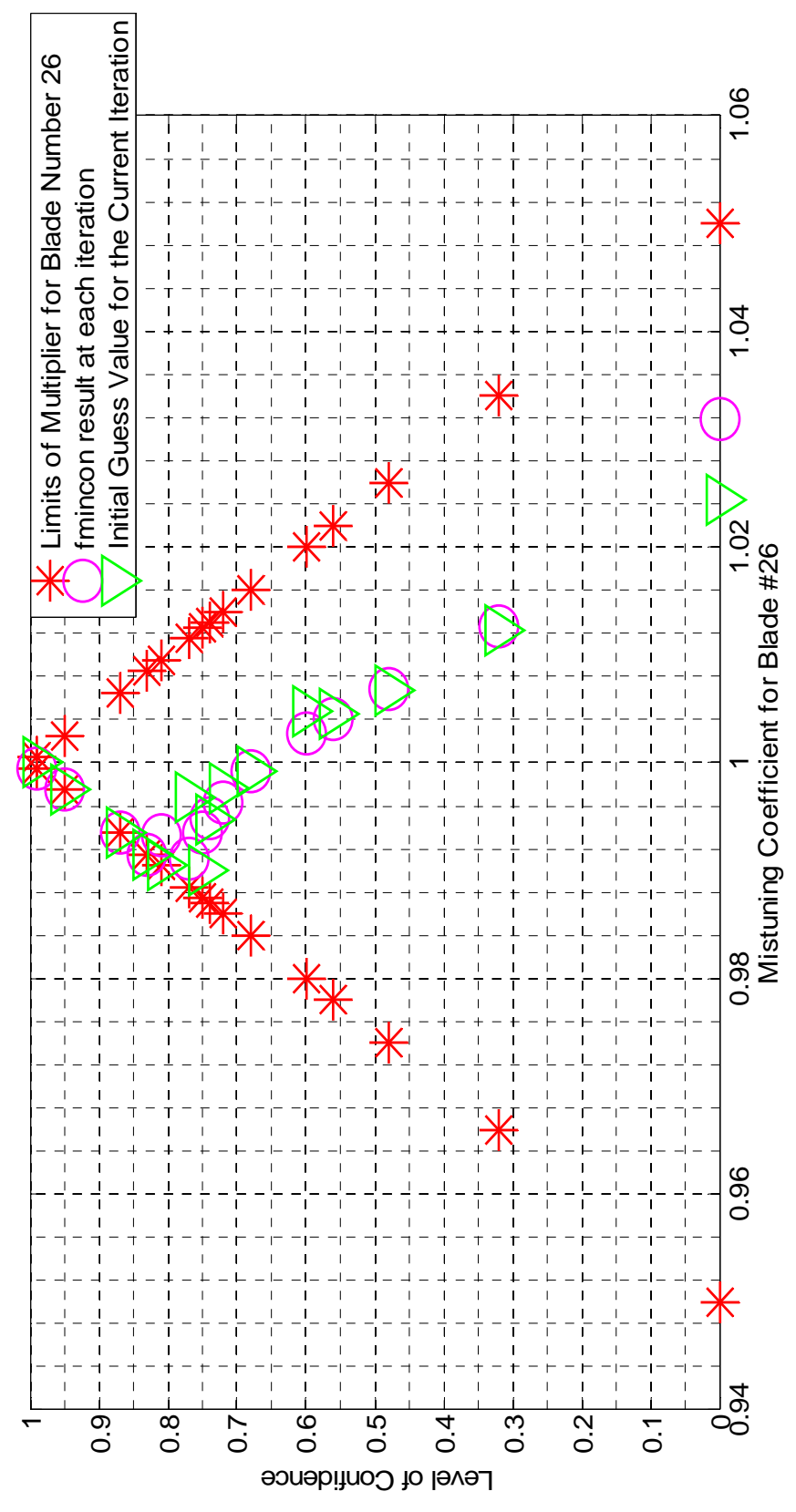

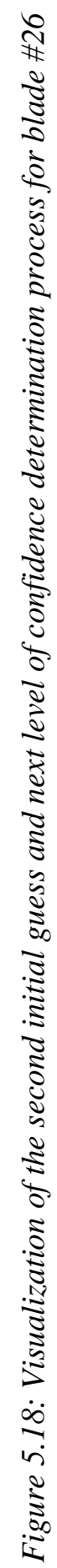




\section{CHAPTER 6}

\section{DISCUSSIONS AND CONCLUSIONS}

In this thesis, use of possibilistic methods on the reliability considerations and specifically on determination of the worst-possible blade forced response in mistuning studies is investigated. The possibilistic uncertainty management tools are adapted to mistuning studies and possibilistic interpretation of mistuning is defined. Consequently, an uncertainty management tool which serves as a more conservative reliability tool and a more accurate and computationally more efficient worst-case determination tool is proposed to be used in mistuned bladed disks studies.

First of all, it is known that, possibilistic analysis handles the imprecision type of uncertainties occurring in engineering systems where there is not enough information about the uncertainty or even the uncertainty is not measurable. It is possible to perform a reliability analysis or determine the worst-case situation by possibilistic methods if only the extent of mistuning deviations can be assigned. Furthermore, in literature, there are two separate methods utilized for reliability analysis and the worst-case determination studies; however, possibilistic analysis enables to conduct both analysis types using the same tool.

Secondly, the proper mathematical formulation for the use of possibilistic methods in mistuning studies is presented. It is found out that the performance of the possibilistic interpretation of mistuning depends on the solution to fuzzy dynamic equation. In this thesis, the extension principle solution to fuzzy equations is used together with the alpha-cut representations of membership function distributions, since the known interval arithmetic methods create dependency issue problem in the solution of fuzzy eigenvalue problem. Interval arithmetic methods are known to be 
faster methods compared to extension principle solutions; hence, especially the computational efficiency of the possibilistic method is open to improvements if an interval arithmetic tool that omits the dependency issue problem is utilized. Unless this is achieved, the only method that can be applied for the determination of the worst case in mistuning studies is the extension principle solution. The approximation to extension principle solution is utilized using the alpha-cut representations of membership function distributions of fuzzy variables and it is found out that the approximation is a valid and efficient approximation in mistuning studies, since bladed disk systems are continuous physical systems. In addition to this, the performance of the extension principle solution is further improved in this thesis. A new worst possible forced response determination method is proposed utilizing homotophy continuation philosophy based on the fact that the physical systems are continuous.

Finally, three case studies are presented in this thesis in order to exemplify and test the performance of the proposed method. The first case study is designed to exemplify the concept of possibilistic interpretation of mistuning by determining the maximum possible forced response values for low, moderate and high reliability levels. In this case study, initially, the worst-possible case among four types of engine order excitations, engine order 1,2,3 and 4, is determined and then, the effects of mistuning on blade forced response amplitudes are investigated for low, moderate and high reliability levels. For the excitation frequency range considered, not all engine order excitations resulted in the energy localization, and for the worst case the maximum amplification factor value is determined as 1.33 .

In the second case study, the possibilistic method (fuzzy modal analysis method) and probabilistic method (Monte-Carlo analysis) are compared in the determination of the worst-case response of a mistuned bladed disk system and in the determination of mistuned natural frequency values. Both methods are compared initially using a simple system having only 2 and 11 uncertain parameters. The results show that as the number of parameters increase, in order to obtain the correct worst case response, the number of iterations in the Monte-Carlo analysis should be increased 
significantly which increases the computational time required drastically. The same comparison is repeated on a lumped parameter bladed disk model with 16 blades considering all 32 stiffness parameters as uncertain. It is observed that increasing the number of iterations in Monte-Carlo analysis from 100 to 500000 decreases the error in the worst-case response from only $\sim 11 \%$ to $\sim 8 \%$. However, utilizing the possibilistic methods, the worst case blade response can be calculated spending significantly less computational time. Moreover, it is observed that the number of uncertain parameters significantly affects the number of iterations required to obtain an accurate worst case blade response. Therefore, in the analysis of a realistic bladed disk system, where the number of uncertain parameters is as well high, using possibilistic methods decreases the computational effort considerably while resulting in the correct solution. Furthermore, from the determined mistuned natural frequency distributions, both methods are observed to be consistent for small mistuning deviations but the difference between the methods becomes more apparent as mistuning increases. The possibilistic method resulted in more conservative mistuned natural frequency distributions compared to probabilistic methods as the range of mistuning increases. Consequently, compatible with the common conclusion in literature given for general design problems, for the mistuned blade problem considered in this work, it is possible to say that probabilistic methods give the mostprobable situations and the possibilistic methods give the worst-possible conditions.

In the final case study, the performances of the possibilistic method and the hybrid algorithm in determining the worst-case mistuned blade forced response are compared. A realistic finite element method is utilized which is constituted by using Craig-Bampton method. Initially, an example where only two blades are assumed to be mistuned is considered. For this example it is possible to evaluate all of the possibilities within the uncertainty range of interest by brutal force; hence it is possible to determine the worst-possible case of the example considered. In this example, possibilistic approach is compared with the genetic algorithm solution which is the first step of the hybrid algorithm. It is observed that the genetic algorithm solution itself does not converge on the global maximum solution even if it 
requires 100 times more computational time compared to the possibilistic approach. Then, the performances of possibilistic methods and hybrid methods are compared on a more realistic case where all of the blades are assumed to be mistuned. Five sample global optimization iterations are continued with the gradient-based optimization algorithm utilizing the solutions of the genetic algorithm as the initial guess. Even if all of the genetic algorithm results were different, it is observed that gradient based optimization for all of them converge on the same mistuned blade forced response value. On the contrary, possibilistic approach is tested by utilizing two different methods which are used for both the determination of the initial guesses and the determination of the next level of confidence. Both of the initial guess and next level of confidence determination processes result in the same mistuned blade maximum forced response value as the hybrid algorithm does. However, the one that determines the next level of confidence based on the number of iterations required at the previous level of confidence spent almost the same amount of time compared to the hybrid algorithm, and the one that determines the next level of confidence depending on the closeness of the initial guess values to the mistuning pattern results in the worst-case situation requires at least 1.8 times less calculation time to converge on the same mistuned blade maximum forced response. The performances of possibilistic methods are subject to change as the option settings used are changed; therefore, several more trials may be done to observe the behavior of possibilistic methods before a generalization is made. In conclusion, for the case considered, the proposed possibilistic method is shown to be a more efficient tool in determining the worst-possible mistuned blade forced response. In this case study, performance of the methods under only $3^{\text {rd }}$ engine order excitation is investigated. The performance of the methods under different engine order excitations may also be studied.

In this thesis, all of the bladed disk systems considered are assumed to be linear systems; therefore, the possibilistic interpretation of mistuning in non-linear bladed disk systems may be investigated in future. Furthermore, in this thesis, only the performance of the possibilistic approach in determining the worst-case situations is investigated. However, it is known that better mistuning configurations are possible 
by utilizing intentional mistuning patterns on bladed disk assemblies, and possibilistic approaches can also be used to find the best mistuning pattern which decreases the maximum blade forced response. 


\section{REFERENCES}

[1] Maglaras, G. ,Nikolaidis, E. , Haftka, R. T., Cudeny, H. H. ( 1997) "Analytical-experimental comparison of probabilistic methods and fuzzy set based methods for designing under uncertainty", Structural Optimization, 13, pp. 69-80.

[2] Whitehead, D.S., "Effect of Mistuning on the Vibration of Turbomachine Blades Induced by Wakes," Journal of Mechanical Engineering Science, vol.8, 1966.

[3] Petrov, E. P., Ewins, D. J. (2003) "Analysis of Worst Mistuning Patterns in Bladed Disk Assemblies", Journal of Turbo Machinery, 125.

[4] Chan, Y.J., Ewins, D.J.,"A Comprehensive Procedure to Estimate the Probability of Extreme Vibration Levels Due to Mistuning", Journal of Engineering for Gas Turbines and Power, Vol.132, 2010.

[5] Chan, Y.J., "Variability of Blade Vibration in Mistuned Bladed Disks", Doctorate Thesis, Impreial College, London, 2009.

[6] Petrov, E.P., "Determination of the Worst Blade Mistuning upon Forced Vibration of Impellers using the Nonlinear Programming Methods", Abstracts of the Conference: Mathematical Simulation of Processes and Structures of Power and Transport Turbines in CAD-CAM Systems, Gotvald, Ukraine, Vol. 3, 1988.

[7] Petrov, E.P., "Optimization of Perturbation Parameters for Forced Vibration Stress Levels of Turbomachine Blade Assemblies", Proceedings of the IUTAM Symposium on Discrete Structural Optimization, Zakopane, Poland, 1993.

[8] Petrov, E.P., lin, S.P., "Search of the Worst and Best Mistuning Patterns for Vibration Amplitudes of Bladed Disks by the Optimization Methods using Sensitivity Coefficients", Proceedings of $1^{\text {st }}$ ASSMO UK Conference Engineering Design Optimization, Ilkley, UK, 1999. 
[9] Petrov, E.P., Vitali, R., Haftka, R., “Optimization of Mistuned Bladed Disks using Gradient Based Surface Approximations", Proceedings of $41^{\text {st }}$ AIAA/ASME/ASCE/AHS/ASC Structures, Structural Dynamics and Materials Conference and Exhibit, Atlanta, USA, Paper AIAA-2000-1522, 2000 .

[10] Scarselli, G., Leece, L.,"Non deterministic approaches for the evaluation of the mistune effects on the rotor dynamics.", AIAA 2004 Conference, California.

[11] Petrov, E.P., Sanliturk, K.Y., Ewins, D.J., "A New Method for Dynamic Analysis of Mistuned Bladed Disks Based on the Exact Relationship Between Tuned and Mistuned Systems", Journal of Engineering for Gas Turbines and Power, Vol.124, 2002.

[12] Rahimi, M., Ziaei-Rad, S., "Uncertainty treatment in forced response calculation of mistuned bladed disks", Mathematics and Computers in Simulation 80, 2010.

[13] Zadeh, L.A.,'Fuzzy Sets as a Basis of a Theory of Possibility", 1999, Fuzzy Sets and Systems 100 Suplement, North-Holland.

[14] Sawyer, J.P., Rao, S.S., "Fuzzy Finite Element Approach for the Analysis of Imprecisely Defined Systems”, AIAA Journal, Vol.33, 1995.

[15] Berke, L., Rao, S.S., "Analysis of Uncertain Structural Systems Using Interval Analysis", AIAA Journal, Vol 35, 1997.

[16] Chen, L., Rao, S.S., "Fuzzy finite-element approach for the vibration analysis of imprecisely-defined systems", Finite Elements in Analysis and Design 27, 1997.

[17] Gersem, H.D., Moens, D., Desmet, W., Vandepitte, D., "Interval and fuzzy dynamic analysis of finite element models with superelements", Computers and Structures 85, 2007.

[18] Moens, D., “A Non-Probabilistic Finite Element Approach for Structural Dynamic Analysis with Uncertain Parameters", PhD Thesis, Katholieke Universiteit Leuven, Mechanical Engineering Department, 2003. 
[19] Moens, D., Vandepitte, D., "An interval finite element approach for the calculation of envelope frequency response functions", International Journal for Numerical Methods in Engineering 61, 2004.

[20] Buckley, J.J., Eslami, E., Feuring, T. "Fuzzy Mathematics in Economics and Engineering”, 2002, Physica-Verlag Heidelberg, New York.

[21] Buckley, J.J., Feuring, T., Hayashi, Y., "Solving Fuzzy Equations using Evolutionary Algorithms and Neural Nets", Soft Computing 6, SpringerVerlag, 2002.

[22] Ciğeroğlu, E , Özgüven, H.N. "Nonlinear vibration analysis of bladed disks by friction dampers", 2006,Journal of Sound and Vibration 295 , 10281043, Elsevier.

[23] Young, J.T., "Primer on the Craig-Bampton Method", Finite Element Modeling Continuous Improvement, NASA, 2000

[24] G. Orbay, "Nonlinear Vibration of Mistuned Bladed Disk Assemblies", MSc Thesis, Middle East Technical University, 2008.

[25] Bladh, J.R., "Efficient Predictions of the Vibratory Response of Mistuned Bladed Disks by Reduced Order Modeling”, PhD Thesis, University of Michigan, 2001.

[26] Biondini, F., Bontempi, F., Malerba, P.G., "Fuzzy Reliability Analysis of Concrete Structures", Computers and Structures, 82, Elsevier, 2004.

[27] Zadeh, L.A., "Fuzzy Sets", 1965, Information and Control 8, 338-353.

[28] Moore, R.E., Kearfott, R.B., Cloud, M.J. "Introduction to Interval Analysis", 2009,Society for Industrial and Applied Mathematics, Philedelphia. 


\section{APPENDIX}

\section{A.PAPERS PUBLISHED}

a) Karataş H.Ç., Cigeroglu E., Özgüven, H. N., "Possibilistic Interpretation of Mistuning in Bladed Disks by Fuzzy Algebra”, IMAC XXX: Conference and Exposition on Structural Dynamics, January 30-February 02, 2012, Jacksonville, Florida, USA.

b) Karataş H.Ç., Cigeroglu E., Özgüven, H. N., "On the comparison of possibilistic and probabilistic methods for the determination of the worst-case response of mistuned bladed disks.", $10^{\text {th }}$ Internation Conference on Vibrations in Rotating Machinery, 11-13 September 2012, Institution of Mechanical Engineers, London. 


\title{
Possibilistic Interpretation of Mistuning in Bladed Disks by Fuzzy Algebra
}

\author{
H. Çăglar KARATAŞ, Ender CİĞEROĞLU, H. Nevzat ÖZGÜVEN \\ Middle East Technical University, Mechanical Engineering Department, \\ 06800, Ankara, Turkey
}

\begin{abstract}
In the study of effect of mistuning on the dynamic response analysis of bladed disk systems, in literature, probabilistic methods are used. Conversely, in this paper, mistuning will be investigated by possibilistic analysis where mistuning parameters are modeled as fuzzy variables possessing possibility distributions. Fuzzy forced response characteristics of mistuned bladed disk systems are determined by mathematical basis of fuzzy sets. In order to do so, extension principle solution of fuzzy functions is used which overcomes the dependency issue problem observed on interval arithmetic solutions; hence, enhancing solution accuracy. Membership function distributions are digitized using alpha-cut methodology, slicing distributions to levels of confidence. Bounds of fuzzy variables in each and every level of confidence are determined using genetic optimization. Using this method, fuzzy forced response characteristics of a cyclically symmetric lumped parameter bladed disk model is determined. The possibilistic interpretation of mistuning is exemplified by determining the bounds of the possible maximum blade forced responses of the system for different orders of engine order excitation and by determining worst-possible case.
\end{abstract}

Keywords: Mistuning, Possibilistic Analysis, Fuzzy Modeling, Maximum Possible Forced Response, Engine Order Excitation, Energy Localization, Extension Principle Solution, Genetic Algorithm.

\section{INTRODUCTION}

Mistuning - deviation from cyclical symmetry in bladed disks - is a phenomenon which results in failure of blades much earlier than their predicted life due to localization of vibration energy on a particular blade. In order to avoid possible failures, the system needs to be analyzed by considering uncertainties in system parameters that are arisen from reasons like manufacturing tolerances, wear in service and material nonuniformities. Depending on the type of analysis, these uncertainties can either be treated as random [6,7] or imprecise. Mathematics and methodologies considering uncertain variables as random variables have already been studied broadly in literature. To the best of authors' knowledge, there is no study on mistuning where the uncertain parameters are treated as imprecise parameters and modeled as fuzzy variables. Therefore, the aim of this paper is to define the possibilistic interpretation of mistuning by explaining the methodology for solving dynamic equations of fuzzy forced response problem which enables the determination of the maximum possible forced response among blades within each and every uncertainty range. 
In order to explain the solution procedure, first of all, details of the process of modeling uncertain variables as fuzzy variables are briefly summarized. Up on the investigation of solution methods to fuzzy equations, proper method is chosen and adapted to fuzzy forced response problems. Finally, the methodology is exemplified by determining the worst-case scenario among different engine order excitations. 


\section{METHODOLOGY}

\subsection{Fuzzy Modeling and Fuzzy Algebra}

For the analysis of engineering systems with vague and imprecise uncertainties, fuzzy set theory and the possibility theory are utilized together. For modeling uncertain parameters as fuzzy variables possessing possibility distributions, possibility theory [2] is used and required mathematical operations regarding fuzzy variables are governed by the fuzzy arithmetic, based on the rules of fuzzy set theory [1], all developed by A. Lutfi Zadeh.

For instance, the proposition $p$ : 'deviation in stiffness parameters of structure $\mathrm{Y}$ is $\pm 5 \%$ at most' carries fuzziness on the values that are going to be assigned to the imprecise variable, deviation in stiffness parameters of structure $\mathrm{Y}, D_{k}$. Due to the fuzziness in meaning of the proposition $p$, the imprecise parameter $D_{k}$ is modeled as fuzzy variable possessing possibility distribution $\Pi_{k}$.

Let us suppose that the universe of discourse $M$ with a generic element $m$, carries all possible values of any stiffness value. Then, the proposition ' $\pm 5 \%$ at most' defines a unique fuzzy subset $F$ which is characterized by the membership function $\mu_{F}$, governing the compatibility of $m$ with the proposition. Namely, regarding to the definition of fuzzy sets [1], every value $m$ takes a membership function value $\mu_{F}(m)$ from 0 to 1 denoting its degree of membership to the fuzzy set $F$ with zero membership function value meaning $m$ does not belong to fuzzy set $F$ and the closer the membership function value to unity the higher the grade of membership of $m$ in $F$. Consequently, the proposition $p$ can be transformed into the proposition " $D_{k}$ is $F$ ".

According to the definition of the concept of possibility distribution [2], the proposition $p$ induces a possibility distribution $\Pi_{k_{\mathrm{Y}}}$ which equates the possibility of $D_{k}$ taking value $m$ to $\mu_{F}(m)$. In this way $D_{k}$ becomes a fuzzy variable which is associated with the possibility distribution $\Pi_{k}$.

For instance, let us suppose that the stiffness parameters are designed to be of value $4 \mathrm{kN} / \mathrm{m}$. Regarding the proposition $p$, stiffness parameters can take values between 3.8 and $4.2 \mathrm{kN} / \mathrm{m}$. Then in order to represent the possibility distribution $\Pi_{k}$ of fuzzy variable $D_{k}$ a triangular fuzzy number $\bar{A}(3.8 / 4 / 4.2)$ can be used as shown in Fig.1;

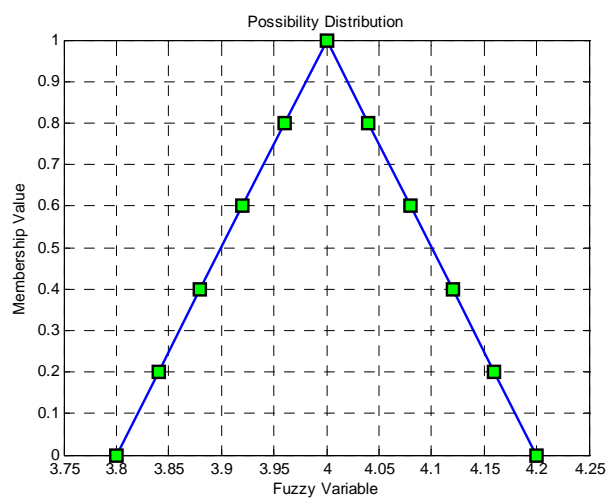

Figure 1: A triangular membership function distribution 
where for a triangular fuzzy number $\bar{A}(x / y / z)$, the three numbers $x<y<z$ identifies the base of the triangular possibility distribution by $[x, z]$ and its vertex by $y$ [3]. Fig. 1 states that the stiffness parameters are designed to be of value $4 \mathrm{kN} / \mathrm{m}$ and they are not expected to be 4.25 or $3.75 \mathrm{kN} / \mathrm{m}$. Furthermore, it is observed that regarding proposition $p$, assessor is more confident to assign $3.9 \mathrm{kN} / \mathrm{m}$ to the stiffness value than $4.15 \mathrm{kN} / \mathrm{m}$ since $3.9 \mathrm{kN} / \mathrm{m}$ has larger possibility value.

Similarly, in this paper, uncertain system parameters of the bladed disk structure are modeled as triangular fuzzy variables possessing $\pm 5 \%$ deviation at most. These fuzzy variables are used in fuzzy forced response analysis in order to obtain worst-possible blade response in blisk.

Mathematically, fuzzy forced response analysis is composed of solutions to fuzzy equations and basic algebraic operations of fuzzy variables. There are two methods for basic algebraic operations of fuzzy variables and solution to fuzzy equations; extension principle solution and alpha-cuts and interval arithmetic solution.

For two fuzzy numbers $\bar{A}$ and $\bar{B}$, if $\bar{A} \bullet \bar{B}=\bar{C}$, $\bullet$ denoting addition, multiplication, subtraction or division, then extension principle defines membership function for $\bar{C}$ as;

$$
\bar{C}(z)=\max _{x, y}\{\min (\bar{A}(x), \bar{B}(y)) \mid x \bullet y=z\}
$$

Extension principle solution, in definition, searches for all $[x, y]_{i}$ pairs that constitute $z_{i}$ and records minimum membership function value within the pairs $\min \left(\bar{A}\left(x_{i}\right), \bar{B}\left(y_{i}\right)\right)$ as $\bar{C}\left(z_{i}\right)$. The membership function value for $\bar{C}\left(z_{i}\right)$ is finally assigned as the maximum among the records, $\max \left(\bar{C}\left(z_{i}\right)\right)$.

In contrast, interval arithmetic method uses alpha-cuts in definition to calculate basic algebraic operations. Alpha-cuts are slices of possibility distributions producing confidence levels as non-fuzzy, crisp intervals, as shown in Fig. 1 by squared marks. For the closed-bounded intervals of alpha-cuts of two fuzzy numbers $\bar{A}$ and $\bar{B}, \bar{A}[\alpha]=\left[a_{1}(\alpha), a_{2}(\alpha)\right]$ and $\bar{B}[\alpha]=\left[b_{1}(\alpha), b_{2}(\alpha)\right]$ (for instance, the least confidence level pf fuzzy variable in Fig. 1 can be written as $\bar{A}[0]=[3.8,4.2]$ ), interval arithmetic solution calculates addition, subtraction, multiplication and division using interval arithmetic rules [4]:

$$
\bar{C}[\alpha]=\bar{A}[\alpha] \square \bar{B}[\alpha]
$$

Due to the rules of interval arithmetic, compared with respect to extension principle solution, larger solution intervals are expected. These rules conflict with the physical structure integrity and creates dependency problem [4]. In contrast, extension principle solution can be adapted to alpha-cut methodology [3]; hence, approximates the true solution practically and without creating dependency issue. Therefore, for the definition of fuzzy functions and for the solutions of fuzzy equations extension principle solution is used in this study.

\subsection{Fuzzy Functions and Solutions of Fuzzy Equations}

Mathematically speaking, fuzzy functions are mapping from fuzzy numbers to fuzzy numbers. They are usually extended from real valued functions. According to extension principle, a real-valued function $h:[x, y] \rightarrow \square$ can be extended to the corresponding fuzzy function $H(\bar{X}, \bar{Y})=\bar{Z}$ as follows:

$$
\bar{Z}(z)=\max _{x, y}\left\{\min (\bar{X}(x), \bar{Y}(y)) \mid h(x, y)=z, a_{1} \leq x \leq b_{1}, a_{2} \leq y \leq b_{2}\right\}
$$

According to Eqn. (3), in order to be able to assess the possibility of the dependent variable $\bar{Z}(z)$, all pairs of the independent variables $[x, y]$ that are mapped into $z$ should be determined regardless of their confidence levels. This makes use of extension principle impossible on computer since it is impossible to determine all the pairs for all types of equations. However, Buckley et.al. [3] proposes that, if the real- 
valued function $h$ is continuous, then upper and lower bounds of the alpha-cuts of fuzzy function $\bar{Z}(z)$ can be determined as follows:

$$
\begin{aligned}
& z_{1}(\alpha)=\min \{h(x, y) \mid x \in \bar{X}[\alpha], y \in \bar{Y}[\alpha]\} \\
& z_{2}(\alpha)=\max \{h(x, y) \mid x \in \bar{X}[\alpha], y \in \bar{Y}[\alpha]\} ; 0 \leq \alpha \leq 1
\end{aligned}
$$

Eqn. (4) uses alpha-cut representation of possibility distributions. For instance, the fuzzy variable in Fig.1 can be expressed in terms of its alpha-cuts as $\bar{A}[\alpha]=[3.8+0.2 \alpha, 4.2-0.2 \alpha], 0 \leq \alpha \leq 1$. So, instead of declaring the shape of the possibility distribution as $\bar{A}(x / y / z)$, the values that share the same confidence level is declared as intervals to represent the fuzzy variable.

By using alpha-cut representation of fuzzy variables on solutions to fuzzy equations, Eqn. (4) approximates the true solution by utilizing independent variables from the same confidence level. Namely, instead of searching all possible independent variable pairs from any confidence level, the ones that share the same possibility distribution value $\bar{X}[\alpha]$ and $\bar{Y}[\alpha]$ are used to determine the interval of dependent variables with the same possibility distribution value, $\bar{Z}[\alpha]$. This approximation makes the use of extension principle possible on computer and hence establishes the basis of solution to fuzzy functions and equations.

In order to determine upper and lower bounds of alpha-cuts of the dependent variable, $\bar{Z}[\alpha]=\left[z_{1}(\alpha), z_{2}(\alpha)\right]$, Eq. (4) requires a minimization and/or maximization operation which is an optimization procedure. Determination of global optimum requires a "good" initial guess if gradient based optimization procedures are used which is not practical; hence, the formulation given above is suitable to be used with genetic algorithm which can be utilized to determine the bounds of the alpha-cuts. In conclusion, in this paper for the solution of fuzzy forced response equations, extension principle is used by utilizing alpha-cut representation of fuzzy variables and by using genetic algorithm for determining bounds of alpha-cuts.

\subsection{Fuzzy Forced Response Analysis}

Mathematically, determining the worst-possible blade forced response requires solution to dynamic equations of fuzzy forced response problem which can be done by modal analysis. For a crisp (non-fuzzy) lumped-parameter system with structural damping, the crisp equation of motion is given as follows:

$$
[M]\{\ddot{q}\}+\left[K^{*}\right]\{q\}=\{F\}
$$

where $\left[K^{*}\right]$ is the complex stiffness matrix including structural damping, $[M]$ is the mass matrix, $\{F\}$ is the forcing vector, $\{q\}$ and $\{\ddot{q}\}$ represents displacement and acceleration vectors, respectively. Since mass and stiffness matrices reflect physical systems, the solution to equation of motion needs to be continuous. Therefore, the equation that is used for determining alpha-cuts of the solution, Eq. (4), is valid.

Consequently, let $\Phi$ be an algorithm that computes the forced vibration responses of the system by modal analysis where it accepts mass and stiffness matrices and forcing vector $[M],\left[K^{*}\right],\{F\}$ as inputs and returns displacement of the nodes of the lumped parameter model $\{q\}$, as the output.

If the system elements are considered as imprecise so as fuzzy variables, the equation of motion transforms into the fuzzy equation of motion:

$$
[\bar{M}]\{\ddot{\bar{q}}\}+\left[\bar{K}^{*}\right]\{\bar{q}\}=\{\bar{F}\}
$$


where over-bar expresses fuzzy matrices and vectors. Then, correspondingly the algorithm $\Phi$ would accept alpha-cuts of elements fuzzy correspondence of the inputs $[\bar{M}],\left[\bar{K}^{*}\right],\{\bar{F}\} ; \bar{m}_{i j}[\alpha], \bar{k}_{i j}[\alpha], \bar{f}_{i}[\alpha]$ and would return the alpha-cuts of elements of fuzzy displacement vector $\{\bar{q}\}, \bar{q}_{i}[\alpha]$ as the output. Then, according to Eqn. (4), assuming the excitation force vector is exactly known, i.e. crisp, alpha-cuts of the blade displacement forced response values with respect to deviations in system parameters are calculated as follows:

$$
\begin{aligned}
& \bar{q}[\alpha]=\left[q_{1}(\alpha), q_{2}(\alpha)\right] \\
& q_{1}(\alpha)=\min \left\{\Phi\left(k_{11}, \ldots, k_{n n}, m_{11}, \ldots, m_{n n}\right) \mid k_{i j} \in \bar{k}_{i j}[\alpha], m_{i j} \in \bar{m}_{i j}[\alpha]\right\} \\
& q_{2}(\alpha)=\max \left\{\Phi\left(k_{11}, \ldots, k_{n n}, m_{11}, \ldots, m_{n n}\right) \mid k_{i j} \in \bar{k}_{i j}[\alpha], m_{i j} \in \bar{m}_{i j}[\alpha]\right\}
\end{aligned}
$$

So, for a vibrating system with imprecise parameters regarding the proposition 'deviation in stiffness parameters of structure is $\pm 5 \%$ at most' can be solved for alpha-cuts of the blade fuzzy forced response elements practically and without creating dependency issue using Eqn. (7).

This concludes explanations to methodology of fuzzy forced response modal analysis. In the following section, given methodology is exemplified by determining the worst-case blade displacement of a cyclically symmetric lumped parameter mistuned bladed disk model under the excitation of an engine order forcing.

\section{NUMERICAL EXAMPLE}

Numerical example considered in this section is designed to exemplify the effects of mistuning on a cyclically symmetric integrated bladed disk system (blisk) by determining the worst-case scenario among different possible engine order excitations. The worst-case scenario for mistuning considerations is the case where the energy is localized on a particular blade. Since energy localization results in much higher displacement values on that particular blade compared to the rest of the blades, the maximum possible blade displacements for each engine order excitation is sought and compared with the rest of the blade displacement values in order to determine the worst-case situation where the energy localization occurs.

Energy localization results from destruction of cyclical symmetry due to uncertainties like manufacturing tolerances, wear in service and material non-uniformities. In this study, these uncertainties are treated as imprecise and assumed to be effecting stiffness parameters only. The stiffness parameters are expected to deviate at most $\pm 5 \%$ from the tuned case stiffness parameters of a cyclically symmetric lumped parameter bladed disk model shown in Fig. 2. 


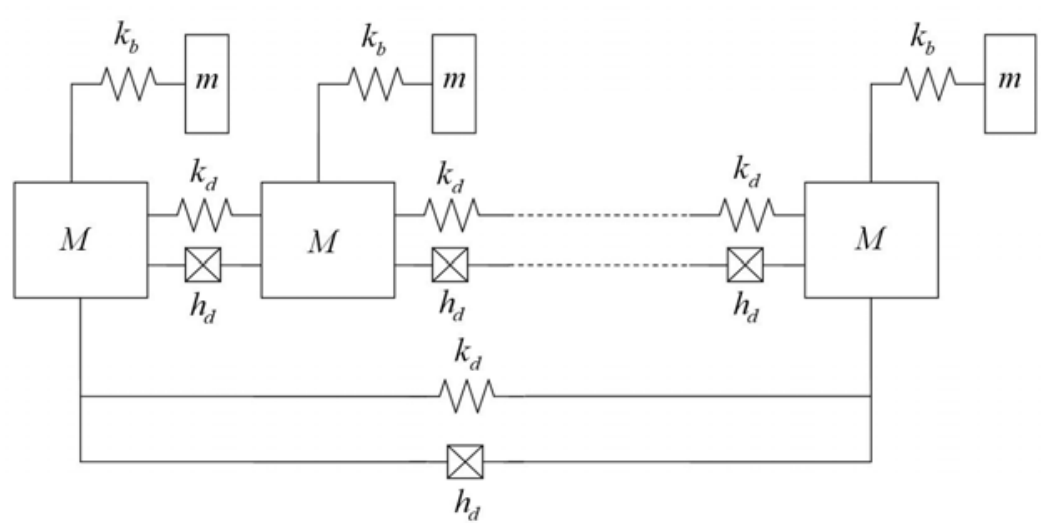

Figure 2: Cyclically Symmetric lumped-parameter jet-engine blisk model

Here, $M, m, k_{d}, k_{b}, h_{d}$ are the disk mass, blade mass, disk stiffness, blade stiffness and structural damping, respectively and $\pm 5 \%$ deviation in imprecise parameter is named as $\pm 5 \%$ mistuning level in this study. The assumption regarding imprecision in system parameters requires fuzzy modeling of stiffness parameters and the use of possibilistic analysis in order to determine maximum possible blade displacement value. Therefore, possibility distributions of fuzzy variables is determined and used in fuzzy forced response analysis using extension principle solution that utilizes alpha-cut methodology and genetic algorithm as stated in Eqn. (7) with fuzzy stiffness parameters $\bar{k}_{d}[\alpha], \bar{k}_{b}[\alpha]$ only.

Let us suppose that elements of blisk model are realized with non-fuzzy, crisp values given in Table 1.

Table 1 : Physical Values of the Lumped Parameter Model

\begin{tabular}{|c|c|}
\hline Parameter & Value \\
\hline Disk Mass, $m_{d}$ & $4 \mathrm{~kg}$ \\
\hline Disk Stiffness, $k_{d}$ & $60000 \mathrm{~N} / \mathrm{m}$ \\
\hline Blade Mass, $m_{b}$ & $2 \mathrm{~kg}$ \\
\hline Blade Stiffness, $k_{b}$ & $7000 \mathrm{~N} / \mathrm{m}$ \\
\hline Structural Damping Coefficient, $\gamma$ & 0.01 \\
\hline
\end{tabular}

All system parameters except stiffness parameters are used in possibilistic analysis with the tuned case values given in Table 1 and stiffness parameters are modeled as triangular fuzzy variables. A triangular possibility distribution has three components, i.e. two base values and a vertex value. For fuzzy stiffness values, tuned case value given is assigned to the vertex of the corresponding fuzzy variable and base values are chosen to be $\pm 5 \%$ deviated from the tuned case value compatible with the assumption, as shown in Fig. 3. 


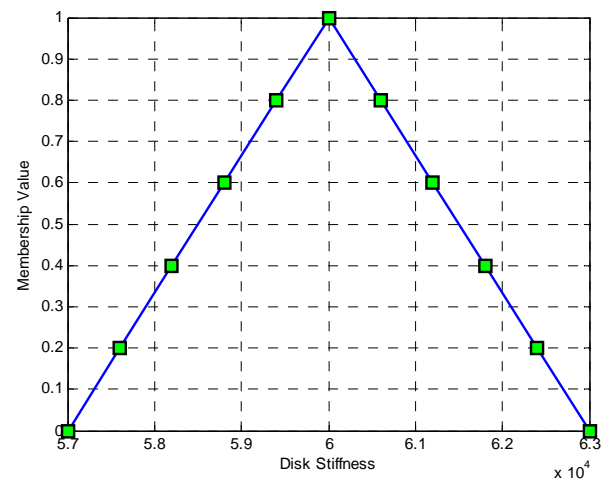

Figure 3 : Possibility distribution for disk stiffnesses

Let us further suppose that the blisk spins in a static pressure field hence experiences engine order excitation which can be modeled as [5]:

$$
f_{j}=F_{m} \cos \left(m \Omega t+\frac{2 \pi m j}{n}\right),
$$

where $j, m, n, \Omega$ and $F_{m}$ are the blade number, engine order, number of blades, rotational speed and excitation amplitude, respectively. The effects of engine orders 1, 2, 3 and 4 of $1 \mathrm{~N}$ forcing amplitude are sought for the worst-case analysis. In order to determine the mode that each type of excitation excites, the frequency term $m \Omega$ is swept within the frequency range of interest. For 0.9 confidence level (corresponding to $\pm 0.5 \%$ mistuning level), at each forcing frequency, the displacement of the blade corresponding to the maximum response is given for different engine order excitations in Fig. 4.

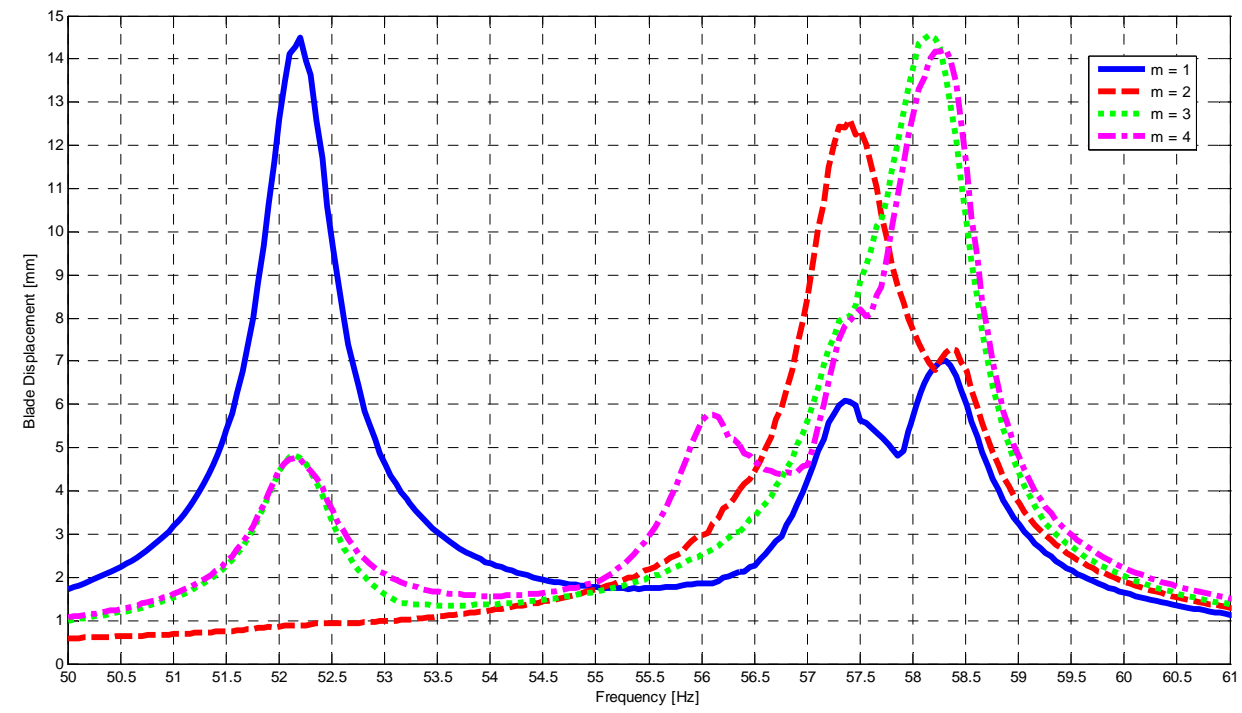

Figure 4: Displacement of the blade corresponding to the maximum response vs. frequency for engine orders $1,2,3$ and $4( \pm 0.5 \%$ mistuning level)

From Fig. 4 it can clearly be seen that different engine order excitations excite different modes of vibration at different resonant frequencies. Also, the figure highlights the maximum possible blade displacements recorded within the blisk for a forcing of $1 \mathrm{~N}$ amplitude and $\pm 0.5 \%$ mistuning level within the forcing frequency range of interest. 
In order to observe the effect of mistuning, together with the blade that has maximum displacement, the vibration amplitudes of rest of the blades are given in Fig. 5 as bar plots for four different engine orders at $\pm 5 \%$ mistuning level.
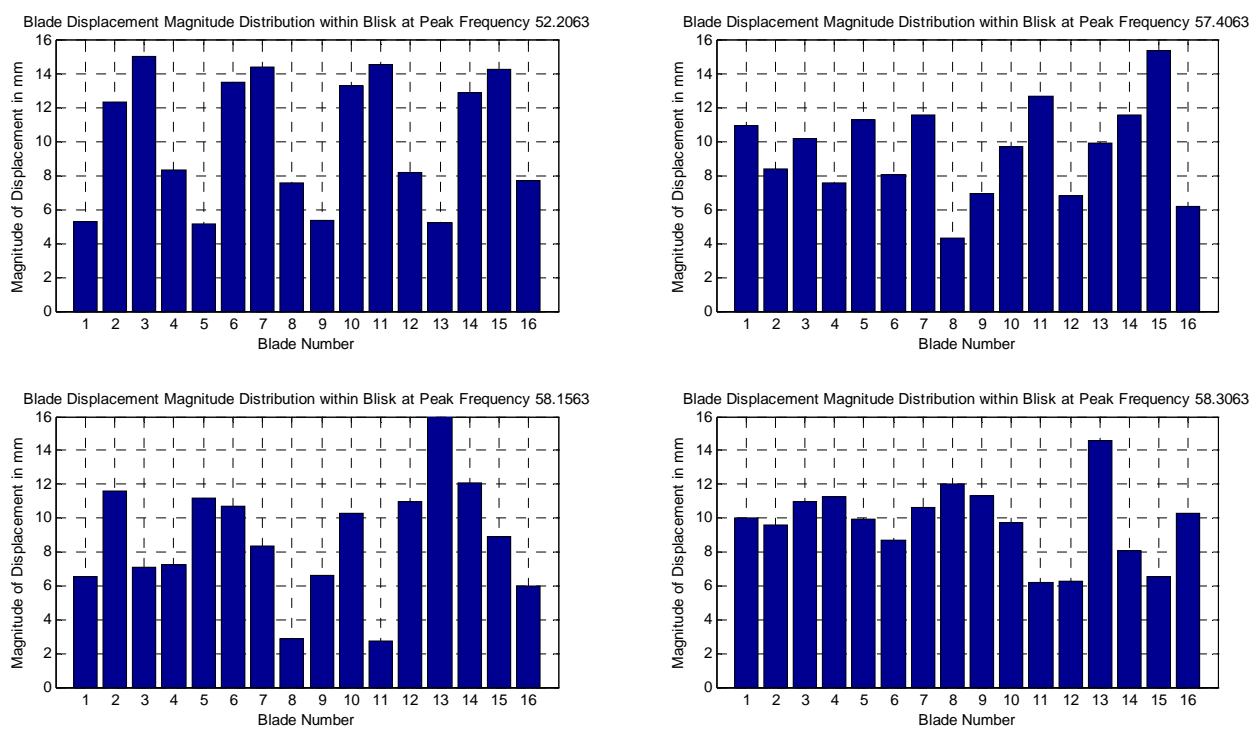

Figure 5: Maximum response of blades for engine orders 1, 2, 3 and 4 ( $\pm 5 \%$ Mistuning Level)

From Fig. 5, among engine orders 1, 2, 3 and 4, the energy localization for engine orders 3 and 4 can clearly be seen in the figure where a single blade has a much larger response compared to all other blades. Moreover, engine order 3 shows the highest maximum blade forced response compared to other cases. Therefore, the worst-possible case is determined to be the case where the blisk is excited by engine order 3 for $\pm 5 \%$ mistuning level.

This concludes the determination of worst-case scenario for the blisk model considered for different engine order excitations. First, the mode that each type of excitation excites is selected by pursuing a frequency sweep within the range of interest and resonant frequencies are determined for $\pm 0.5 \%$ mistuning level since different engine order excitations excite different modes of vibration. Then the behavior of the blisk excited by four different engine orders at the corresponding resonant frequencies is investigated and the case where the blisk is excited by engine order 3 is determined to be the worst-possible case for the system considered. Furthermore, In Fig. 6, behavior of the blisk excited by engine order 3 for different mistuning levels is detailed at different excitation frequencies. The plot contains representative mistuning levels to show the change in the maximum blade displacement with respect to increasing percent deviations in stiffness parameters. It is observed that the possibility of obtaining a higher maximum forced response increases as the mistuning level increases. Therefore, it can be concluded that around the crisp resonant frequency, the possibility of resonance increases as the mistuning level increases. 


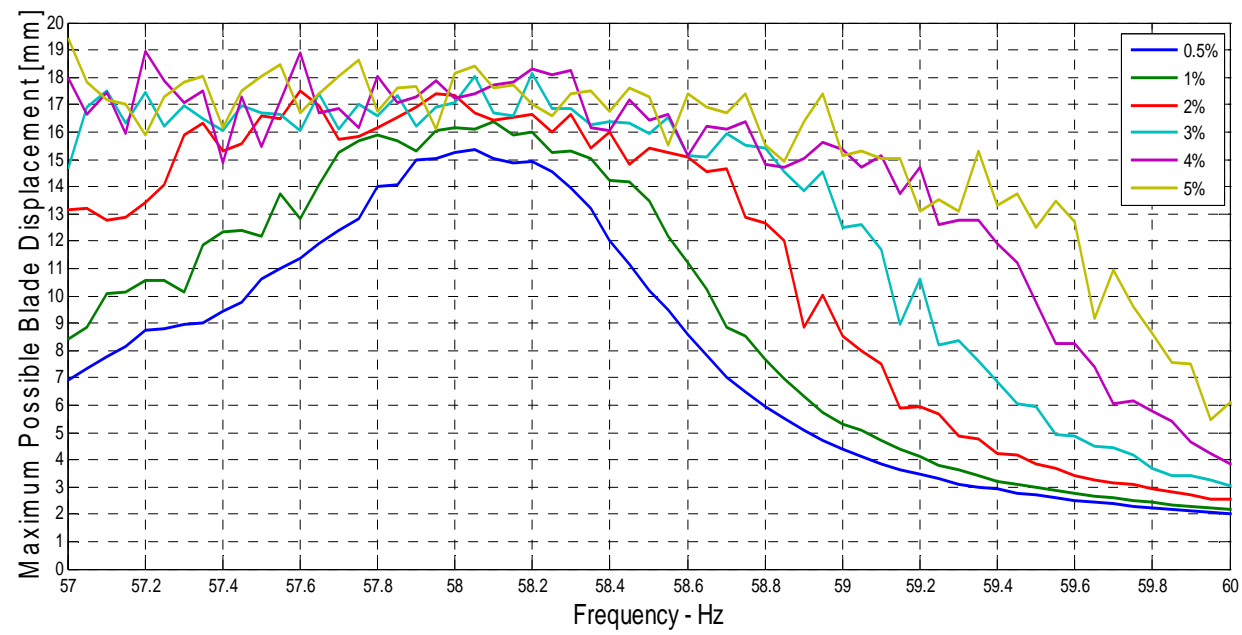

Figure 6: Maximum blade displacement as a function of frequency and mistuning level (Engine order 3 )

\section{CONCLUSION}

In conclusion, this study explains and exemplifies the methodology to treat uncertainties in mistuning problems as imprecise and the way to pursue fuzzy forced response analysis. The proper fuzzy arithmetic method is suggested in order to overcome the dependency issue problem. Adapting fuzzy solution tools to modal analysis, solution to fuzzy forced response problem is proposed. Using fuzzy forced response analysis that uses extension principle solution which utilizes alpha-cut methodology and genetic algorithm, the worst-possible effect of mistuning on a cyclically symmetric lumped parameter model that is excited by different engine order excitations is determined. It is observed that not all engine order excitations result in energy localization. Therefore, in order to determine the critical one, several engine order excitations are required to be checked separately. Finally, the trend of change of maximum blade displacement with respect to changes in frequency and mistuning level is obtained. It is concluded that possibility of resonance increases as the mistuning level increases around the tuned case resonant frequency.

\section{REFERENCES}

[1] Zadeh, L.A., "Fuzzy Sets", 1965, Information and Control 8, 338-353.

[2] Zadeh, L.A.,"Fuzzy Sets as a Basis of a Theory of Possibility”, 1999, Fuzzy Sets and Systems 100 Suplement, North-Holland.

[3] Buckley, J.J., Eslami, E., Feuring, T. “ Fuzzy Mathematics in Economics and Engineering”, 2002, Physica-Verlag Heidelberg, New York.

[4] Moore, R.E., Kearfott, R.B., Cloud, M.J. “Introduction to Interval Analysis”, 2009,Society for Industrial and Applied Mathematics, Philedelphia.

[5] Ciğeroğlu, E , Özgüven, H.N. "Nonlinear vibration analysis of bladed disks by friction dampers", 2006, Journal of Sound and Vibration 295, 1028-1043, Elsevier.

[6] J. H. Griffin and T. M. Hoosac, "Model development and statistical investigation of turbine blade mistuning," 1984,Journal of Vibration, Acoustics, Stress, and Reliability in Design, vol. 106, no. 2, pp. 204-210. 
[7] A. Sinha, "Calculating the statistics of forced response of a mistuned bladed disk assembly," 1986, AIAA Journal, vol. 24, no. 11, pp. 1797-1801. 


\title{
On the comparison of possibilistic and probabilistic methods for the determination of the worst-case response of mistuned bladed disks
}

\author{
H Ç Karataş, E Cigeroglu, H N Özgüven \\ Middle East Technical University, Mechanical Engineering Department, \\ 06800, Ankara, Turkey
}

\begin{abstract}
In this paper, the possibilistic and probabilistic methods are compared with each other by utilizing a lumped parameter vibratory system and a mistuned bladed disk model. In these models stiffness elements are considered to be uncertain, which are assumed to have Gaussian probability distribution. Both methods are utilized for determining uncertain natural frequencies and the worst-case blade tip displacements. The blade tip displacement amplitudes gathered from different number of Monte-Carlo iterations and calculated by fuzzy forced response solution are compared and it is found that among the two, only the possibilistic method converges to the worst-possible blade tip displacement value, and requires significantly less computational time.
\end{abstract}

\section{INTRODUCTION}

Mistuning which occurs from destruction of cyclical symmetry in bladed disk assemblies due to residual uncertainties is a well-studied phenomenon in vibrations of rotating machinery. It has been investigated under several sub-topics in engineering discipline; such as identification, determining the worst and the best mistuning pattern, forced response reduction and structural health monitoring. The performance of all these investigations boils down to the true quantification of uncertainties that are present in the engineering system [1-4]. In this paper, performances of the two approaches to handle uncertainties; i.e. probabilistic and 
possibilistic methods are compared for their reliability and computational effectiveness on determining the worst-case scenarios of bladed disk assemblies.

The favorable areas for the two interchangeable methods have been investigated by several researchers and Maglaras et.al. [5] reviewed the state of the art in literature. In the review paper, the authors stated the common conclusion that probabilistic methods favor in situations where there is enough information regarding uncertainties, and possibilistic methods favor in situations where there is lack of information and in situations where the worst-case scenarios are sought. In this paper, a conclusion parallel to the common conclusion in literature is drawn by utilizing Monte-Carlo methods for probabilistic analysis and fuzzy modal analysis method for possibilistic analysis for the same problem. Possibilistic methods are found to be more reliable and computationally more efficient in determining the uncertainty range of the worst-case natural frequency and the worst-possible forced response of a mistuned bladed disk model at each and every confidence level.

The methodology that is used to compare reliability and computational performances of fuzzy modal analysis and Monte-Carlo analysis on determining the worst-case response is detailed in the next section and it has been exemplified by two case studies. In the case studies, a simple lumped parameter system and a cyclically symmetric lumped parameter bladed disk model is used to interpret the progress of the reliability performances of the methods. The value of the worst-case blade response is calculated for uncertain stiffness parameters in order to compare the computational effectiveness of the methods.

\section{METHODOLOGY}

In this study, methods that characterize the same uncertain situation in two different ways are compared with each other on the basis of reliability and computational efficiency in determining the worst-case situations in bladed disk assemblies. Probabilistic methods assume uncertainties to be random where the outputs of an uncertain event are precisely measurable but cannot be determined before an experiment is conducted. These methods model uncertain variables as random variables and characterize uncertainties by probability density functions. In this method, expected values of outcomes of uncertain situation are interpreted by processing relative frequency of outputs obtained for the input random set. On the other hand, possibilistic methods are used to account for another type of uncertainty called imprecision which assumes either precision of measurements to be very low or meaning of measurements to be not clear. In this method, uncertain parameters are modeled as fuzzy variables and characterized by possibility distributions [6]. In possibilistic analysis, range of possible values of outcomes are sought and organized for each and every confidence level. 
The connection between possibilistic and probabilistic methods are established upon the logical truth stating that 'probability of a measured event cannot be more than possibility of that event' [5] which is based on possibility/probability consistency theorem proposed by Zadeh [7]. Due to nature of the theorem, interpretation of the connection remains open to variations according to situation at hand. In this paper, uncertain parameters are assumed to deviate around design value of corresponding parameter in the engineering system and also deviations of possible values of uncertain variables are assumed to be bounded. According to this assumption, the design value of the uncertain parameter needs to be assigned to the mean value of the random set that have Gaussian distribution for probabilistic analysis and membership function distribution value of 1 for possibilistic analysis. The assumption further necessitates both distribution functions to share the same uncertainty bounds, as shown in Fig. 1 below.

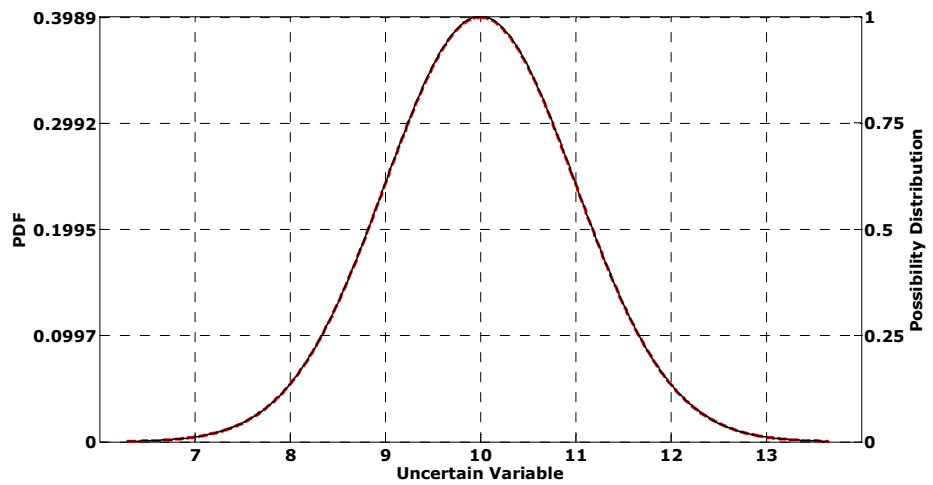

Figure 1: Consistency of the two uncertainty models

The assumption establishes the connection by stating the most expected values and the bounds of uncertainty ranges, only. In order to determine states in remaining levels of confidence, a distribution character of uncertainties needs to be assessed. In this paper, compatible with the mistuned bladed disk systems, stiffness parameters are assumed to have Gaussian distribution characters that is identified by standard deviations and mean values. According to the assumption, design values of stiffness parameters are assigned to the mean value of the corresponding Gaussian distributions and the extent of deviations of uncertain parameters around the design values are represented by assigned standard deviation values.

In probabilistic analysis Gaussian random sets containing values of uncertain stiffness variables are used in Monte-Carlo analysis in order to come-up with random sets of mistuned natural frequencies and values of blade forced responses. Monte-Carlo analysis picks one value from each 
random set of stiffness variables in each iteration and calculates mistuned outputs by modal analysis. Pursuing iterations for a number of times, creates a random set for each output. The probability density functions of calculated (random) mistuned natural frequencies are used for comparison with the corresponding possibility distributions on the basis of calculation of the uncertainty ranges corresponding to the worstcase natural frequency. Moreover, the worst-case forced response value is determined from the random data set of mistuned forced responses.

Correspondingly in possibilistic analysis, in order to come up with possibility distribution functions of mistuned natural frequencies and the worst-possible blade responses, intervals of confidence levels of possibility distributions (alpha-cuts) are used in extension principle solution of the fuzzy modal analysis. Alpha-cuts are slices of the possibility distributions which assesses expected values that a fuzzy variable can take within the corresponding level of confidence. For instance, for a fuzzy stiffness parameter $\bar{k}$, alpha-cut of 0.4 confidence level is represented by an interval $\bar{k}[0.4]=\left[k_{\text {lower }}, k_{\text {upper }}\right]$ meaning that at 0.4

confidence level, stiffness parameter $\bar{k}$ is expected to take values between $k_{\text {lower }}$ and $k_{\text {upper }}$. Here, alpha-cuts of possibility distributions of uncertain stiffness parameters are determined from the corresponding probability density functions compatible with the interpretation of consistency principle in this study, which is expressed mathematically as follows

$$
\mu(x)=\frac{p(x)}{\max [p(x)]},
$$

where $\mu(x)$ stands for membership function distribution of the possibility distribution and $p(x)$ stands for the probability density function. Eq. (1) scales probability density functions with its maximum value resulting in a membership value of 1 , where both distributions share the same uncertainty bounds. Furthermore, utilizing Eq. (1), alpha-cuts of possibility distributions of fuzzy stiffness variables, used in fuzzy modal analysis, are calculated, an example of which is shown in Fig. 2. It should be noted that, possibilistic method does not require a prior knowledge of distribution of the uncertainty; however, in order to perform a valid comparison the same distribution as in the probabilistic method is used in this study. 


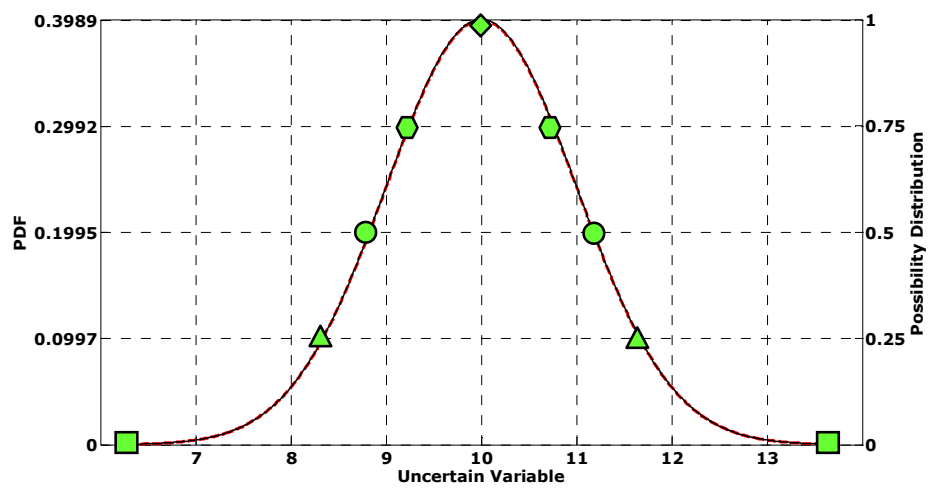

Figure 2: Alpha-cuts of possibility distributions

Alpha-cuts of fuzzy stiffness parameters are processed in extension principle solution of fuzzy modal analysis in order to determine the alpha-cuts of the fuzzy mistuned natural frequencies and the worstpossible blade forced responses within the uncertainty range of interest. Extension principle solution of fuzzy equations is a mapping between inputs and outputs of a fuzzy function that arranges possibility value of the output with respect to the possibility values of the input. It looks for each and every pair of inputs that constitute an output -regardless of their confidence levels- and sets the possibility value of the output accordingly. However, since the physical systems are continuous, in the solution of the worst-case problems, a special case of the extension principle method for the continuous functions is used. This method searches for all pairs of inputs that constitute an output in the same confidence level and assigns the possibility value of the output to possibility value of the inputs $[6,8]$.

The formulation of extension principle solution for fuzzy natural frequency and fuzzy forced vibration responses are given as follows.

$$
\begin{aligned}
& \bar{\omega}[\alpha]=\left[\omega_{1}(\alpha), \omega_{2}(\alpha)\right], \mathrm{n}=1 \ldots \mathrm{DOF} \text { of the lumped parameter system } \\
& \omega_{1}(\alpha)=\min \left\{\Psi\left(k_{11}, \ldots, k_{n n}\right) \mid k_{i j} \in \bar{k}_{i j}[\alpha]\right\} \\
& \omega_{2}(\alpha)=\max \left\{\Psi\left(k_{11}, \ldots, k_{n n}\right) \mid k_{i j} \in \bar{k}_{i j}[\alpha]\right\} \\
& \bar{q}[\alpha]=\left[q_{1}(\alpha), q_{2}(\alpha)\right] \\
& q_{1}(\alpha)=\min \left\{\Phi\left(k_{11}, \ldots, k_{n n}\right) \mid k_{i j} \in \bar{k}_{i j}[\alpha]\right\} \\
& q_{2}(\alpha)=\max \left\{\Phi\left(k_{11}, \ldots, k_{n n}\right) \mid k_{i j} \in \bar{k}_{i j}[\alpha]\right\}
\end{aligned}
$$

In the above formulation, the algorithm $\Psi$ is utilized to calculate alphacuts of fuzzy natural frequency, $\bar{\omega}[\alpha]$, which accepts alpha-cuts of fuzzy 
stiffness variables $\bar{k}_{i j}[\alpha]$ and returns the upper and the lower bounds of fuzzy natural frequencies $\left[\omega_{1}(\alpha), \omega_{2}(\alpha)\right]$ at the same confidence level. Likewise, the algorithm $\Phi$ calculates alpha-cuts of fuzzy forced response which accepts again alpha-cuts of fuzzy stiffness variables $\bar{k}_{i j}[\alpha]$ and returns the upper and the lower bounds of fuzzy forced response $\left[q_{1}(\alpha), q_{2}(\alpha)\right]$. These algorithms utilize modal analysis methods and use genetic algorithm in order to process $\mathrm{min} / \mathrm{max}$ operations.

The ensemble of alpha-cuts of fuzzy natural frequencies constitutes the possibility distribution of the fuzzy natural frequencies and the resulting possibility distribution character is compared with character of the probability distribution obtained by probabilistic analysis for reliability considerations. Furthermore, the upper bound of the fuzzy forced response, i.e. parameter $q_{2}(\alpha)$, gives the worst-possible blade forced response in each and every confidence level, and the upper bound obtained for the least confidence level is compared with the corresponding maximum blade forced response value calculated from Monte-Carlo simulations.

The use of alpha-cuts in possibilistic methods brings computational advantage, since the calculations can be done iteratively by using the results of the previous level of confidence in the next level of confidence. This opportunity results in an increase in computational accuracy and a decrease in computational time. Together with the reliability based comparison, the advantages of utilization of alpha-cuts are exemplified on two lumped parameter systems in this study. First, a simple lumped parameter system with different number of uncertain variables is used to show the performance of the methods with increasing number of uncertain parameters. Afterwards, a lumped parameter model of a mistuned bladed disk system is considered and the worst-case mistuning considerations are exemplified utilizing all stiffness parameters as uncertain.

\section{NUMERICAL EXAMPLES}

\subsection{CASE I - Simplest Case}

The case is designed to exemplify the similarities and differences between both methods by considering different number of uncertain variables. The case utilizes a 10 degree-of-freedom (dof) lumped parameter model as the vibrating system which is excited by sinusoidal excitations. The layout of the 10 dof system is shown in Fig. 3 and the design values of mass and stiffness parameters are given in Table 1 and

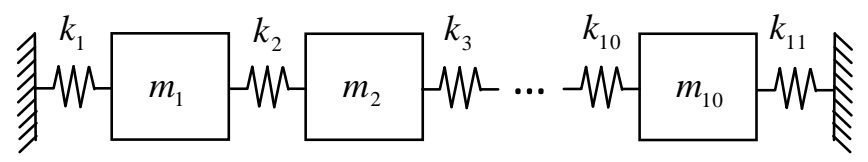


a structural damping ratio of 0.01 is used in the analysis.

Figure 3: 10 dof lumped parameter model

Table 1: Design Values of 10 dof Lumped Parameter System

\begin{tabular}{|l|c|c|c|c|c|c|c|c|c|c|c|}
\hline Parameter & $m_{1}$ & $m_{2}$ & $m_{3}$ & $m_{4}$ & $m_{5}$ & $m_{6}$ & $m_{7}$ & $m_{8}$ & $m_{9}$ & $m_{10}$ & \\
\hline Value [kg] & 2,88 & 3,45 & 3,74 & 7,21 & 3,40 & 7,92 & 3,39 & 4,59 & 7,89 & 3,93 \\
\hline Parameter & $k_{1}$ & $k_{2}$ & $k_{3}$ & $k_{4}$ & $k_{5}$ & $k_{6}$ & $k_{7}$ & $k_{8}$ & $k_{9}$ & $k_{10}$ & $k_{11}$ \\
\hline $\begin{array}{l}\text { Value } \\
\text { [N/m] }\end{array}$ & 43687 & 32816 & 46975 & 45701 & 30577 & 41973 & 46461 & 43431 & 35511 & 44366 & 31767 \\
\hline
\end{tabular}

The mass and stiffness values given Table 1 are determined random normal distribution around a mean of $2 \mathrm{~kg}$ and $30000 \mathrm{~N} / \mathrm{m}$, respectively. The system is excited by two sinusoidal excitations of $100 \mathrm{~N}$ and $200 \mathrm{~N}$ amplitudes with an excitation frequency of $24.52 \mathrm{~Hz}$ (first natural frequency value) at the first and the second degrees-of-freedoms respectively.

In the first part of this case study, values of the stiffness parameters $k_{1}$ and $k_{2}$ are assumed to be uncertain and have Gaussian distribution where parameters deviate around their design values with $2 \%$ standard deviation which corresponds to at most $\pm 8.5 \%$ deviation from the design value. In the second part of this case study, all stiffness parameters are assumed to be uncertain having a normal distribution with a standard deviation of $2 \%$.

\subsection{CASE II - Worst-Case Scenarios in Bladed Disk Assemblies}

The second case study is designed to compare performances of the methods on a cyclically symmetric structure, i.e. a lumped parameter bladed disk model. Both methods are applied to determine the worstcase scenarios occurring due to mistuning phenomenon. In a mistuned bladed disk structure, the mistuning is specified by the deviation of mistuned natural frequencies from the tuned natural frequencies; therefore, on the design stage it is important to determine the worstpossible ranges of frequency deviation for reliability considerations at each and every confidence level. Furthermore, it is known that mistuning results in localization of vibration energy on a particular blade; hence, 
the worst-possible vibration amplitude amplification value is sought in order to determine the extent of mistuning effect on the mistuned structure. In both of these investigations, not only the accuracy, but also computational time requirements are important for engineering purposes.

A cyclically symmetric lumped parameter model with 16 blades shown in Fig. 4 is used as the bladed disk model in the second case study. The disk mass, $M$, disk stiffness, $k_{d}$, blade mass, $m$ and blade stiffness, $k_{b}$, are given in Table 2, below. All 32 stiffness values are assumed to deviate around their design value given in Table 2 with a standard deviation of $0.15 \%$ which corresponds to at most $\pm 0.8 \%$ deviation from the design values. The same bladed disk system with $\pm 5 \%$ at most has already been investigated by the authors in a previous study [6] where the bladed disk was assumed to spin in a static pressure field under the effect of the first four engine order excitations. In that study, the third engine order excitation is determined to be the worst case in the frequency range of [57-59] $\mathrm{Hz}$. Since the stiffness values of the bladeddisk model considered here are assumed to deviate at most $\pm 0.8 \%$, which is one sixth of the one in [6], instead of investigating the frequency range of 57 to $59 \mathrm{~Hz}$, response of the system at only a single frequency is considered. This excitation frequency is $57.87 \mathrm{~Hz}$ corresponding to the ninth resonant frequency of the tuned model. Third engine order excitation is considered in the analysis, which is determined to be the worst case for the system studied [6].

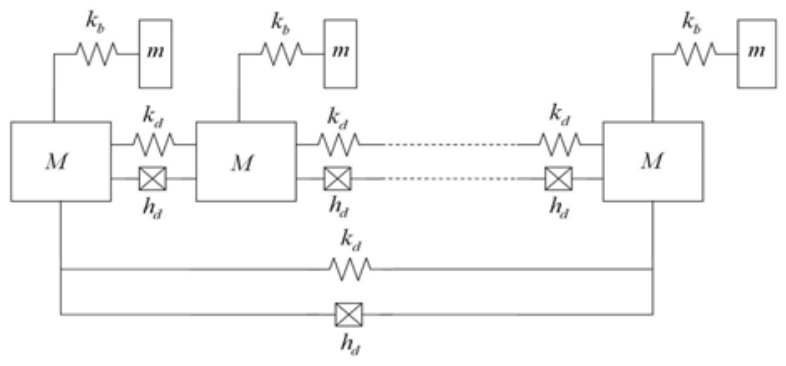

Figure 4: Cyclically Symmetric Lumped Parameter Model of a Bladed Disk System

Table 2: Physical Values of the Cyclically Symmetric Lumped Parameter Model

\begin{tabular}{|c|c|}
\hline Parameter & Value \\
\hline Disk Mass & $4 \mathrm{~kg}$ \\
\hline Disk Stiffness & $60000 \mathrm{~N} / \mathrm{m}$ \\
\hline
\end{tabular}




\begin{tabular}{|c|c|}
\hline Blade Mass & $2 \mathrm{~kg}$ \\
\hline Blade Stiffness & $7000 \mathrm{~N} / \mathrm{m}$ \\
\hline Structural Damping Coefficient & 0.01 \\
\hline
\end{tabular}

\section{RESULTS AND DISCUSSIONS}

The two case studies are used to exemplify a consistent and a deviated situation on a theoretical model and the behavior of the methods on a model of real-world structure. In all these studies, two important mistuning parameters; the worst-case uncertainty range of mistuned natural frequencies and the worst-case forced response values are sought. The worst-case uncertainty ranges of mistuned natural frequencies are represented by the probability density functions (pdf) in probabilistic analysis and by the possibility distributions in possibilistic analysis. The computed uncertainty ranges in each and every confidence level is obtained by plotting the upper and lower values found on the same abscissa, as shown in Fig. 5 below.

Fig. 5 represents the performance of the methods on a simple theoretical lumped parameter model with two uncertain stiffness values as explained in Case I. It is observed that, in this case, possibilistic and probabilistic methods converges on the same uncertainty ranges. However, this result cannot be generalized, since the performance of the methods deviates as the number of uncertain stiffness parameters increase as shown in Fig. 6 below.

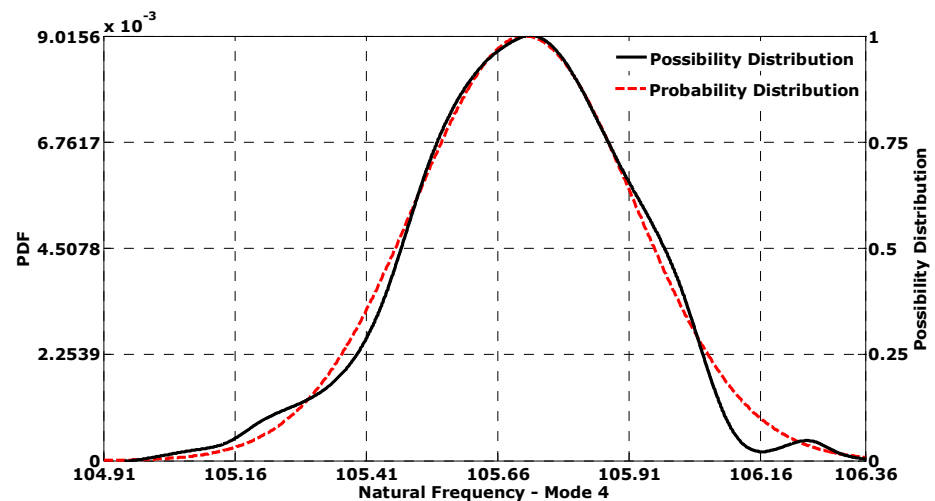

Figure 5: Simple Lumped Parameter System $4^{\text {th }}$ Natural Frequency Distribution- Case I First Part 


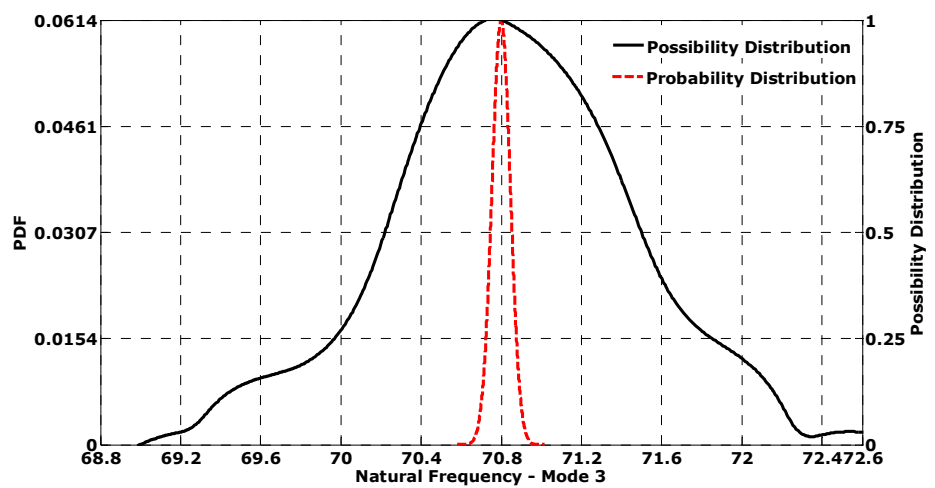

Figure 6: Simple Lumped Parameter System $3^{\text {rd }}$ Natural Frequency Distributions - Case I Second part

From the results given in Fig. 6 it is observed that the possibilistic method gives larger uncertainty ranges at each confidence level compared to the probabilistic method. Therefore, possibilistic methods are found to be more conservative and more reliable in the determination of the worst case. Correspondingly, it can be concluded that probabilistic methods are suitable for the determination of the most probable result whereas possibilistic methods should be used for the identification of the worstpossible case.

A parallel conclusion can be drawn by investigating the performances of the methods in the calculation of the worst-possible forced response values. The results for Case I obtained by fuzzy modal analysis solution and Monte-Carlo analysis with different number of iterations are compared in Fig. 7.

7a) 


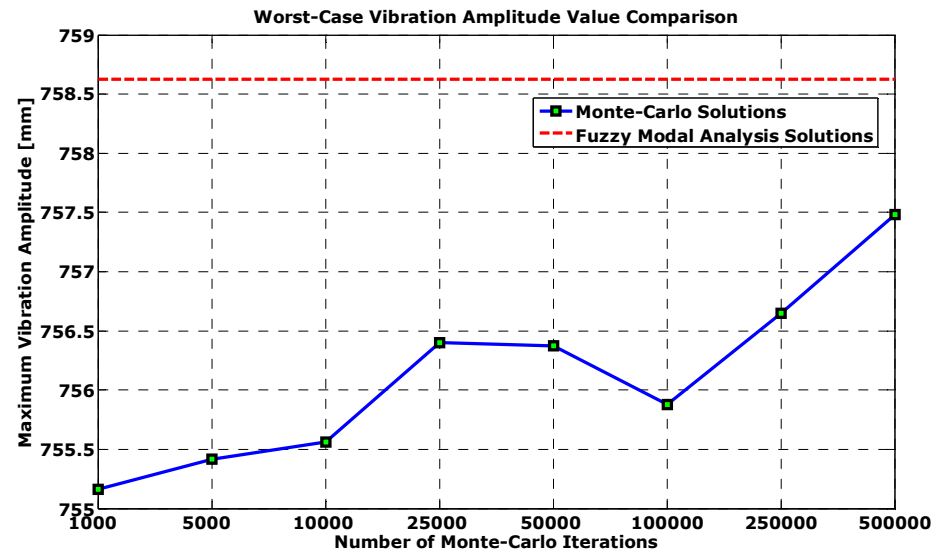

7b)

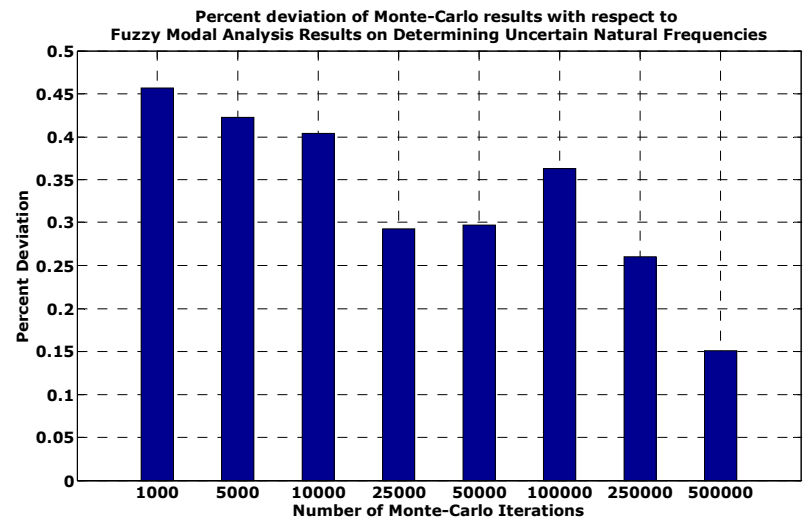

7c) 


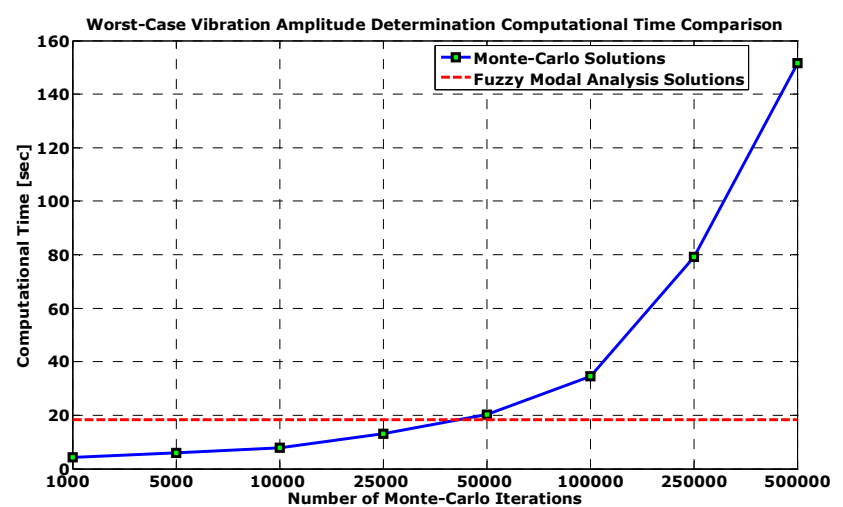

7d)

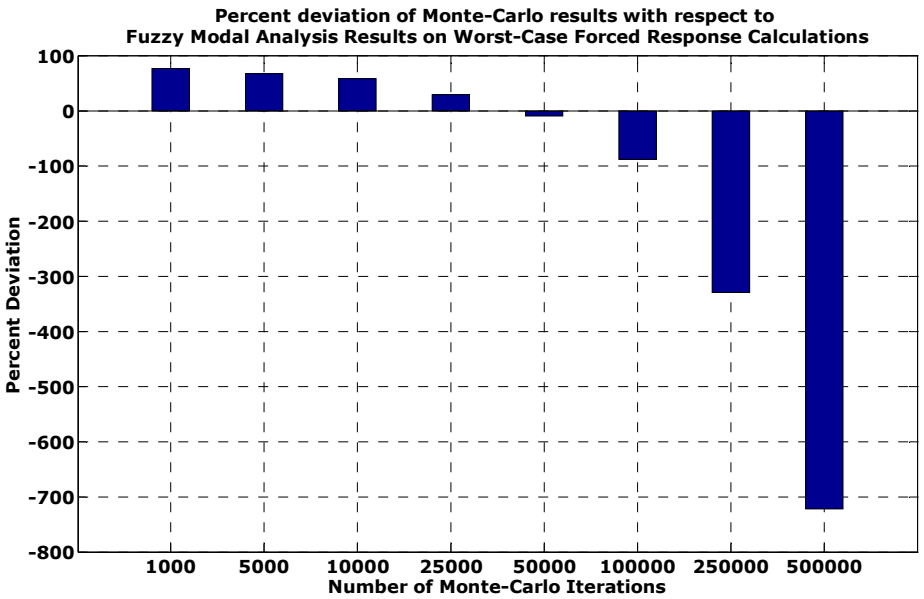

Figure 7: Computational Efficiency Comparison - Case I First Part

From Fig. 7, it is easy to observe that for the system explained in Case I, probabilistic and possibilistic methods can result in the same worst-case forced response value. However, as the number of uncertain parameters increases, the possibilistic methods results in the worst-possible response requiring significantly less computational effort, as shown in Fig. 8. More importantly, increasing the number of iterations in the Monte-Carlo analysis does not improve the results significantly. The error between 100 to 500000 iterations lies approximately at 5\%, whereas the computational time required increases more than 700 times compared to the fuzzy modal analysis. 
8a)

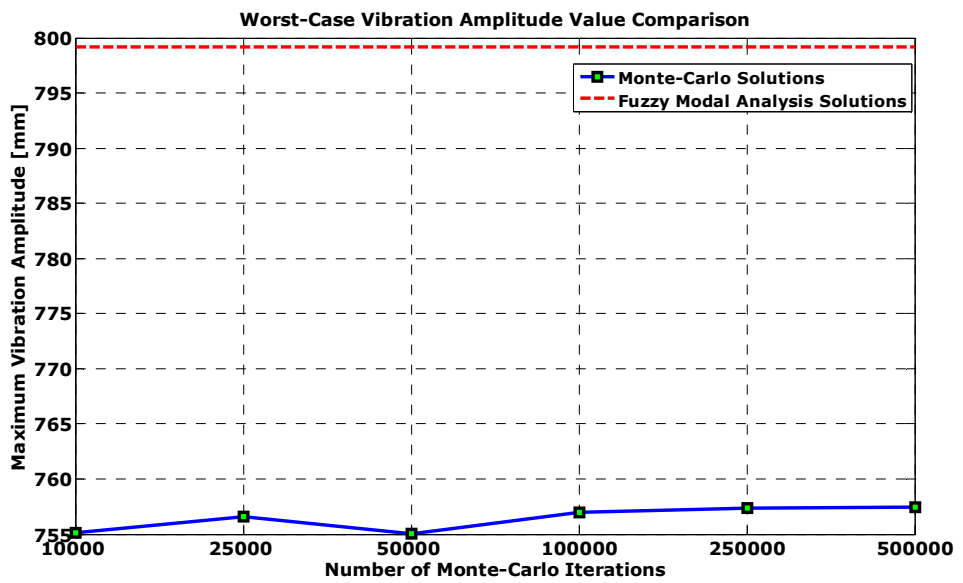

8b)

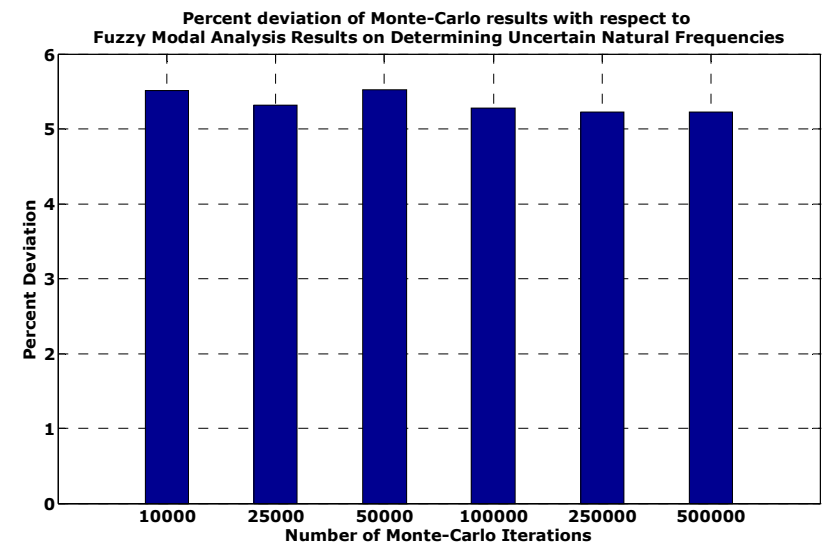

8c) 


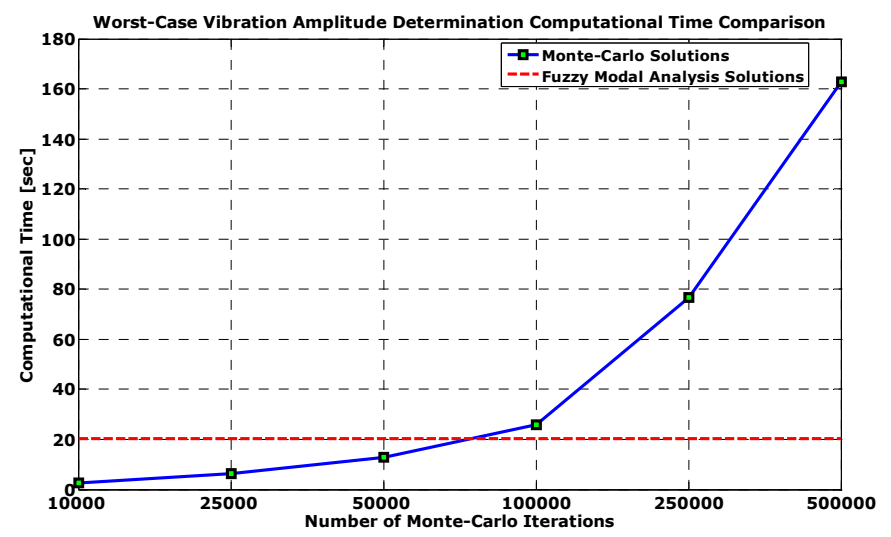

8d)

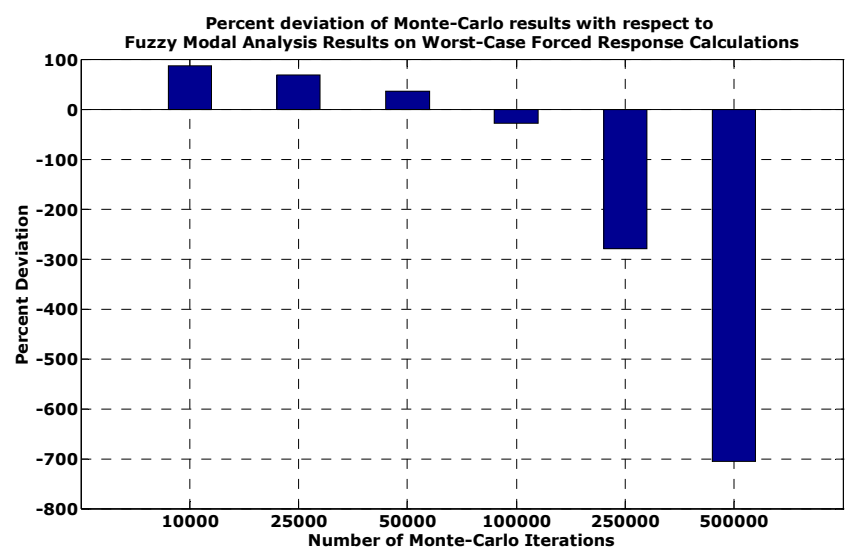

Figure 8: Computational Efficiency Comparison - Case I Second Part

In the final case study, the same analyses are repeated on a bladed disk system with 16-blades. The reliability and computational efficiency comparison results are given in Fig. 9 and Fig 10 below. It is observed that, for the bladed disk system, even though the standard deviation is much smaller than the first case study, the error between the probabilistic and possiblistic methods is higher in the range of $11 \%$ to $8 \%$. Moreover, increasing the number of iterations in Monte-Carlo analysis does not result in a significant improvement in the value of the worst response whereas the computational effort required increases more than 6000 times. This is due to the fact that the number of uncertain parameters is nearly 3 times of the first case study. 


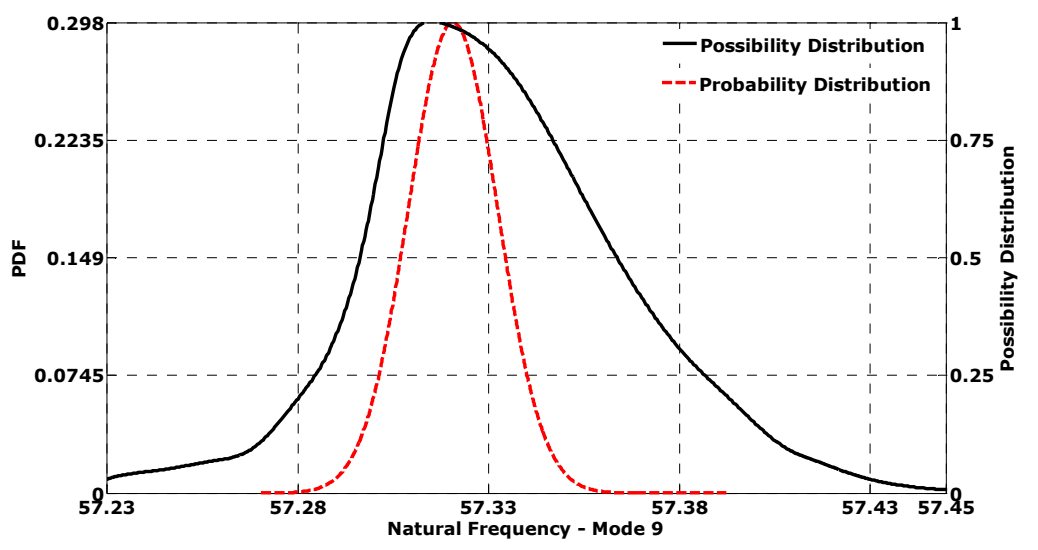

Figure 9: Mistuned Bladed Disk Model $9^{\text {th }}$ Natural Frequency Distribution

10a)

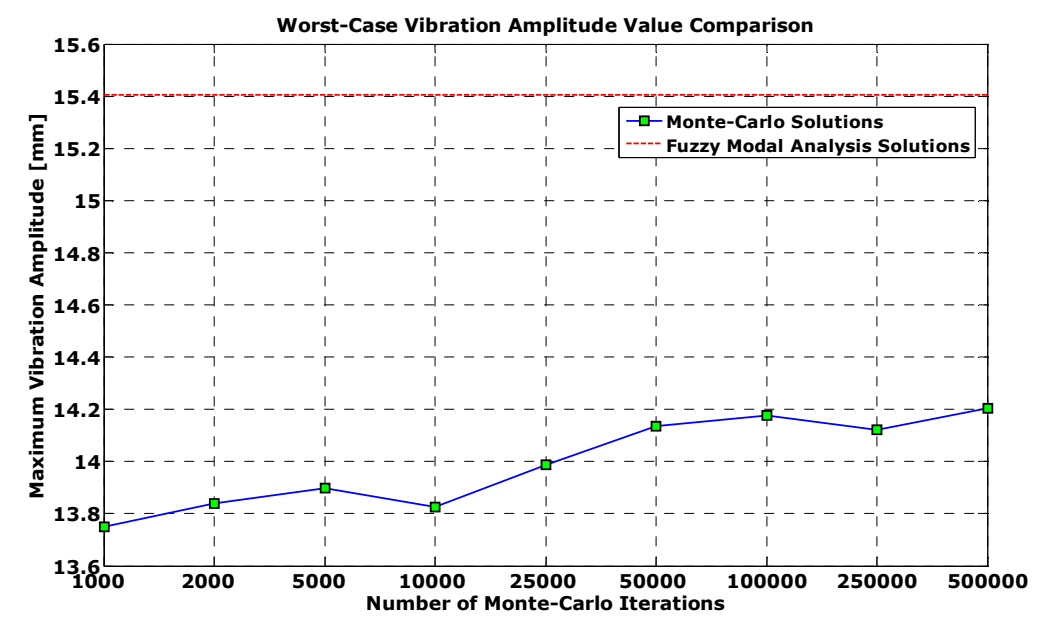

10b) 


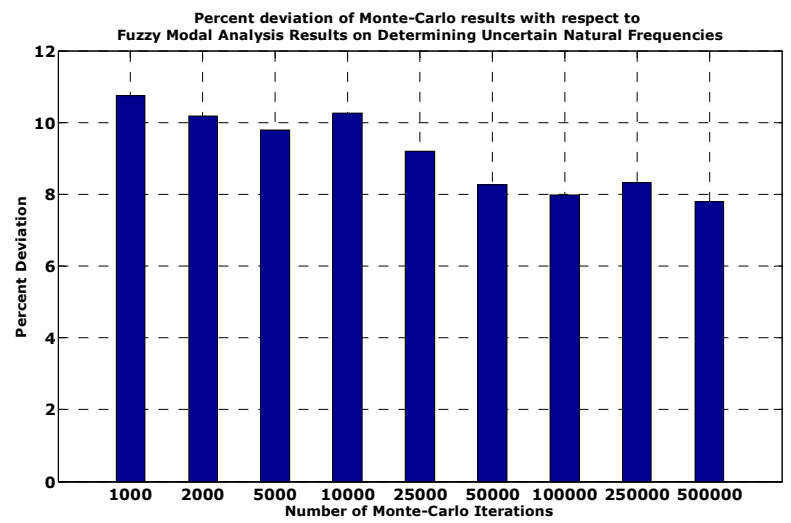

10c)

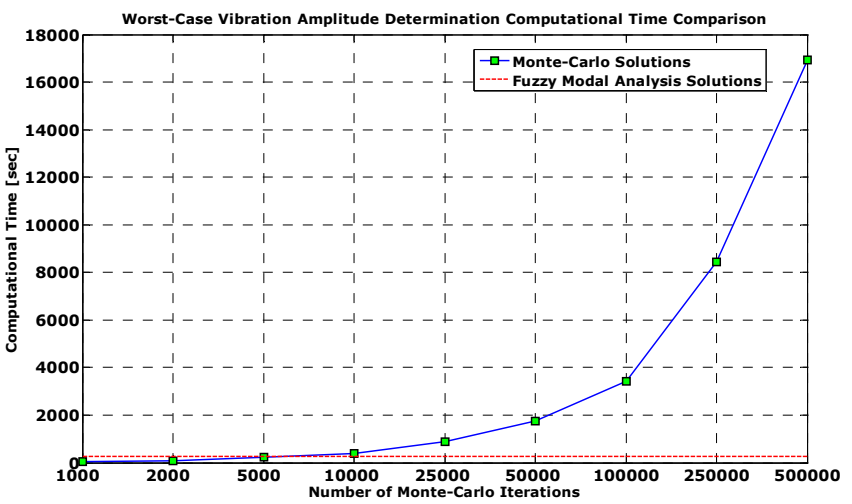

10d) 


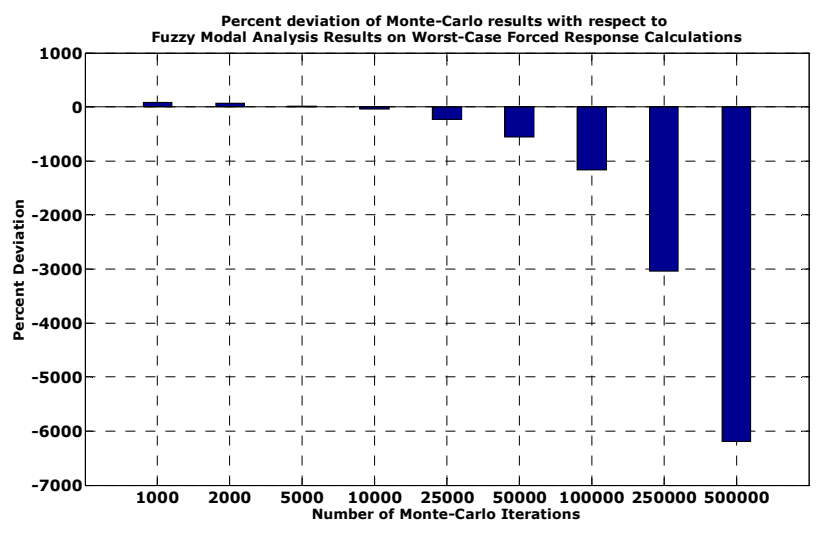

Figure 10: Computational Efficiency Comparison - Cyclically Symmetric Case

\section{CONCLUSIONS}

In this paper, the possibilistic method (fuzzy modal analysis method) and probabilistic method (Monte-Carlo analysis) are compared in the determination of the worst-case response of a mistuned bladed disk system. Both methods are compared initially using a simple system having only 2 and 11 uncertain parameters. The results show that as the number of parameters increase, in order to obtain the correct worst case response, the number of iterations in the Monte-Carlo analysis should be increased significantly which increases the computational time required drastically. The same comparison is repeated on a lumped parameter bladed disk model with 16 blades considering all 32 stiffness parameters as uncertain. It is observed that increasing the number of iterations in Monte-Carlo analysis from 100- 500000 decreases the error in the worstcase response from $\sim 11 \%$ to $\sim 8 \%$. However, utilizing the possibilistic methods, the worst case blade response can be obtained requiring significantly less computational time. Moreover, it is observed that the number of uncertain parameters significantly affects the number of iterations required to obtain an accurate worst case blade response. Therefore, in the analysis of a realistic bladed disk system, where the number of uncertain parameters is as well high, using possibilistic methods decreases the computational effort significantly while resulting in the correct solution.

\section{REFERENCES}

[1] Petrov, E. P., Ewins, D. J. (2003) "Analysis of Worst Mistuning Patterns in Bladed Disk Assemblies", J ournal of Turbo Machinery, 125. 
[2] J. H. Griffin and T. M. Hoosac (1984) "Model development and statistical investigation of turbine blade mistuning", Journal of Vibration, Acoustics, Stress, and Reliability in Design, 106 (2), pp. 204-210.

[3] Sinha A. (1986) "Calculating the statistics of forced response of a mistuned bladed disk assembly", AlAA Journal, 24 (11), pp. 1797-1801.

[4] Liao, H., Wang, J., Yao, J., Li, Q. (2010) "Mistuning Forced Response Characteristics Analysis of Mistuned Bladed Disks", J ournal of Gas Turbines Power, 132.

[5] Maglaras, G. ,Nikolaidis, E. , Haftka, R. T., Cudeny, H. H. ( 1997) "Analytical-experimental comparison of probabilistic methods and fuzzy set based methods for designing under uncertainty", Structural Optimization, 13, pp. 69-80,

[6] Karataş H. Ç., Cigeroglu E., Özgüven H. N., "Possibilistic Interpretation of Mistuning in Bladed Disks by Fuzzy Algebra", IMAC XXX: Conference \& Exposition on Structural Dynamics, January 30-February 02, 2012, Jacksonville, Florida, USA.

[7] Zadeh, L. A. (1999) "Fuzzy Sets as a Basis of a Theory of Possibility", Fuzzy Sets and Systems 100 Suplement, North-Holland.

[8] Buckley, J.J., Eslami, E., Feuring, T. (2002) " Fuzzy Mathematics in Economics and Engineering", Physica-Verlag Heidelberg, New York. 